\title{
PUPILLARY LIGHT REFLEX IN CHILDREN WITH AUTISM SPECTRUM DISORDERS
}

\author{
A Dissertation \\ presented to \\ the Faculty of the Graduate School \\ at the University of Missouri-Columbia \\ In Partial Fulfillment \\ of the Requirements for the Degree \\ Doctor of Philosophy \\ by \\ CHATHURI DALUWATTE \\ Dr. Gang Yao, Dissertation Supervisor \\ MAY 2013
}


The undersigned, appointed by the dean of the Graduate School, have examined the dissertation entitled

\section{PUPILLARY LIGHT REFLEX IN CHILDREN WITH AUTISM SPECTRUM DISORDERS}

presented by Chathuri Daluwatte,

a candidate for the degree of Doctor of Philosophy,

and hereby certify that, in their opinion, it is worthy of acceptance.

Dr. Gang Yao, Department of Biological Engineering

Dr. Judith H. Miles, Thompson Center for Autism \& Neurodevelopmental Disorders

Dr. Shawn Christ, Department of Psychological Sciences, Thompson Center for Autism \& Neurodevelopmental Disorders

Dr. Shinghua Ding, Department of Biological Engineering

Dr. John Viator, Department of Biological Engineering 
To Ammi... 


\section{ACKNOWLEDGEMENTS}

I wish to express my heartiest gratitude to Dr. Gang Yao, who has served as my advisor during the past four and half years. The inspiration he has been setting was a major driving force throughout my research and I will forever be grateful to Dr. Yao for the challenging way he improved my logical thinking, scientific methodology and professionalism.

My deep appreciation is expressed to Dr. Judith $\mathrm{H}$. Miles, for her warmest encouragements, insights and all the guidance throughout the research. She has been a great inspiration for me. I am grateful to Dr. Shawn Christ and Dr. David Beversdorf for their valuable contributions which tremendously improved the quality of this research. I would also like to express my gratitude for Jill Akers and Nicole Takahashi for their amazing job in recruiting and coordinating such a large number of research participants. Had it not been for them this research would not have been possible. I also want to express my warmest gratitude for Andrew Lofgreen and Nathan Berliner for their support in recruiting the large group of control participants and their help in image processing and data entry. I am also indebted to my dissertation committee members Dr. John Viator and Dr. Shinghua Ding for their valuable advice.

I am deeply grateful to Dr. Xiaofei Fan who supported me during the early stage of the study to get a smooth start with the project. I am thankful for my colleagues in our research group, Dr. Chuanmao Fan, Randima Dinalankara and Ben Davis for the fruitful discussions and support and above all for their friendship. I would like to 
especially be thankful to Dr. Ali Shauib who generously helped me a lot during the testing stages of the research.

I am deeply thankful to my best friend Awanthi Hewage, without whose support and unshaken friendship I would not have made it through graduate school.

Ultimately, I am grateful for the love and support of my mother, who has been the source of strength in my life. I sincerely appreciate all her support and patience which is the sole reason for my success. 


\section{TABLE OF CONTENTS}

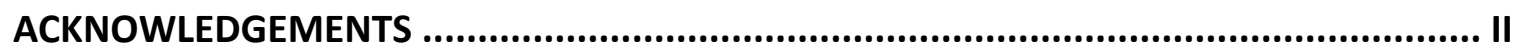

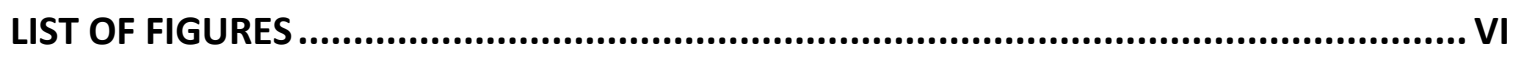

LIST OF TABLES ..................................................................................................... VIII

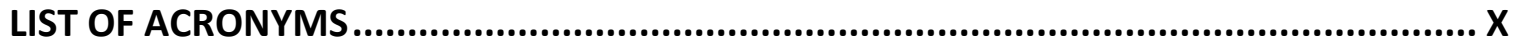

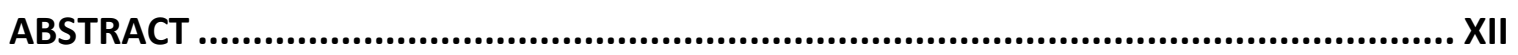

Chapter Page

1 INTRODUCTION............................................................................................ 1

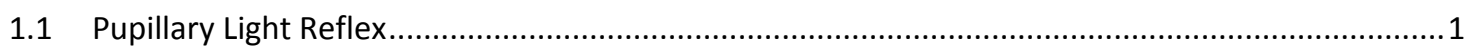

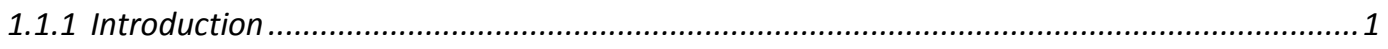

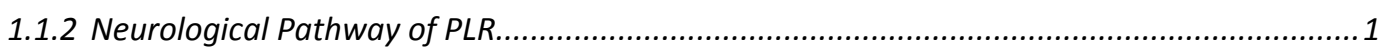

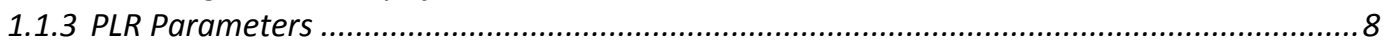

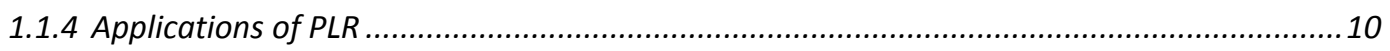

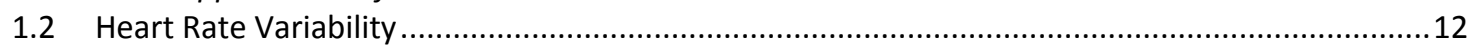

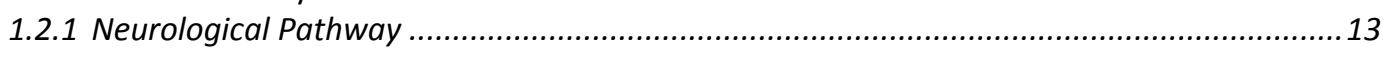

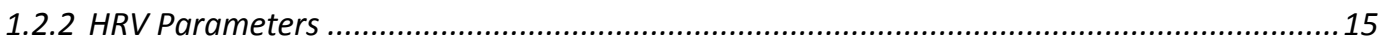

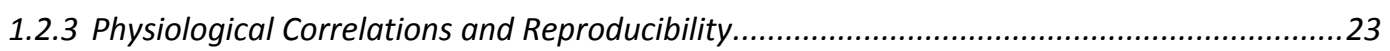

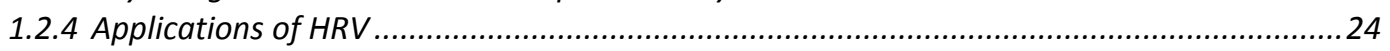

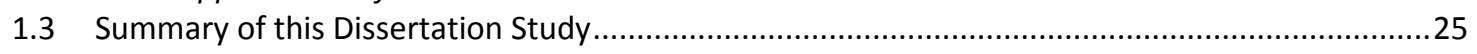

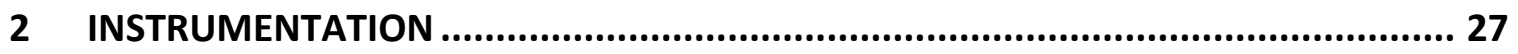

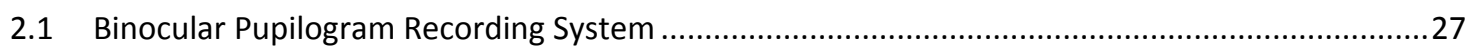

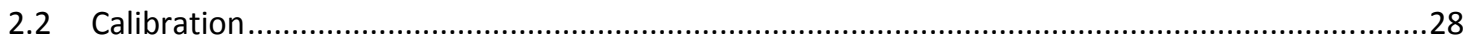

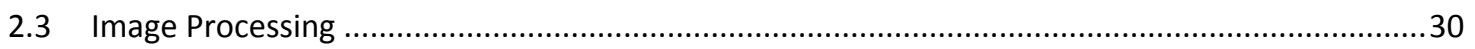

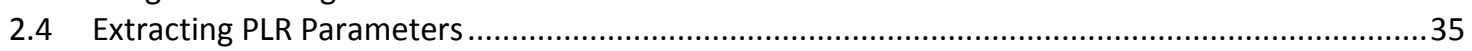

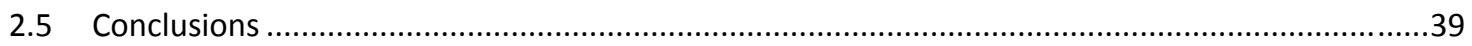

3 SIMULTANEOUSLY MEASURED PUPILLARY LIGHT REFLEX AND HEART RATE

VARIABILITY IN HEALTHY CHILDREN............................................................... 41

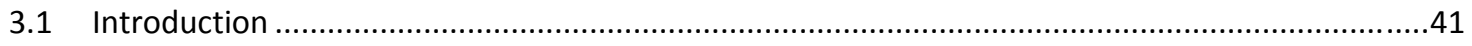

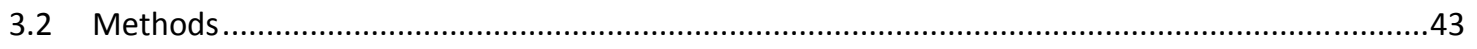

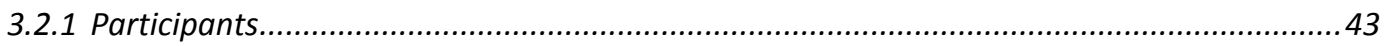

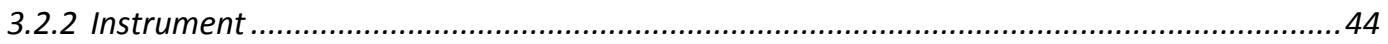

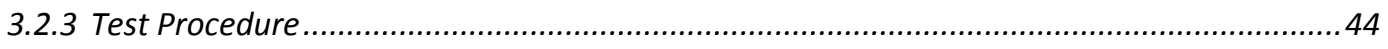

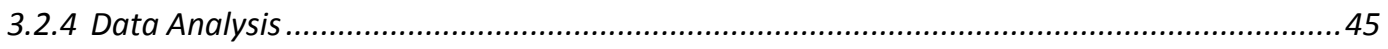

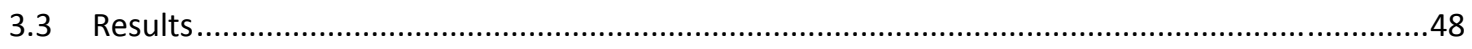

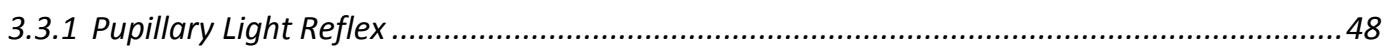

3.3.2 Heart Rate Variability ............................................................................................. 49

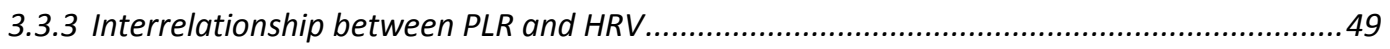

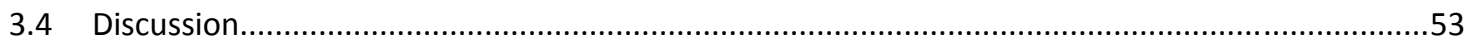

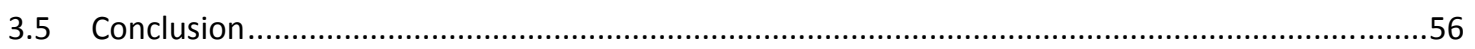




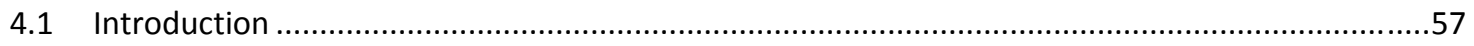

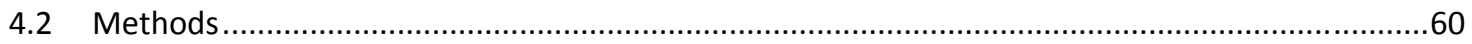

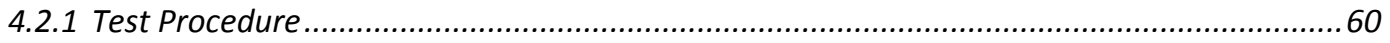

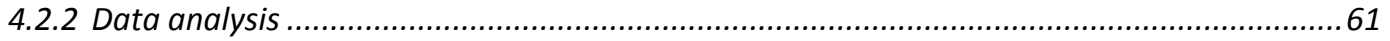

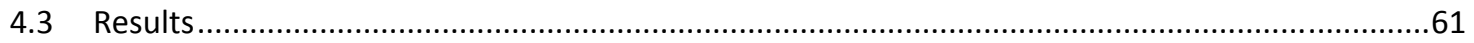

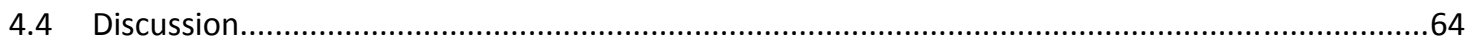

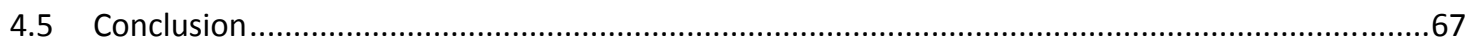

5 ATYPICAL PUPILLARY LIGHT REFLEX AND HEART RATE VARIABILITY IN CHILDREN WITH AUTISM SPECTRUM DISORDERS............................................. 68

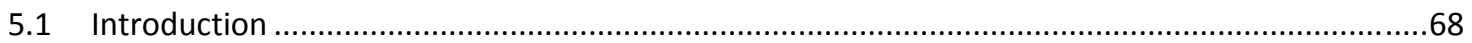

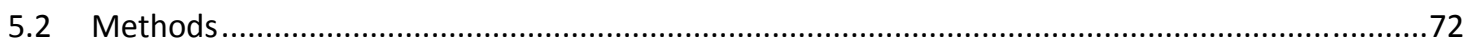

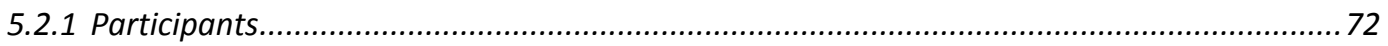

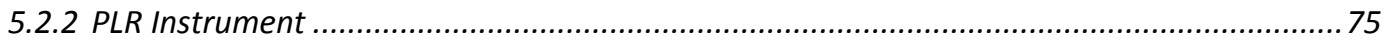

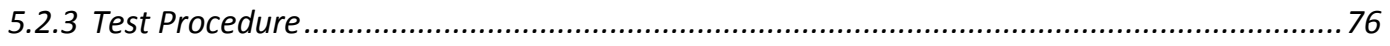

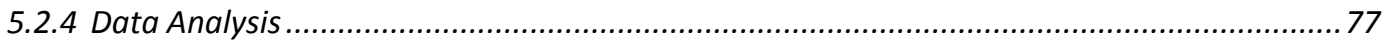

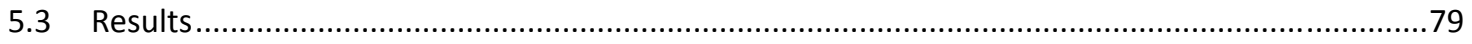

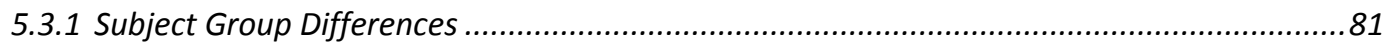

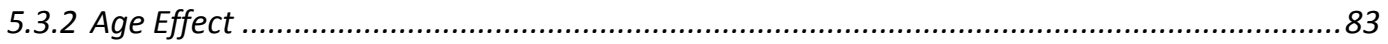

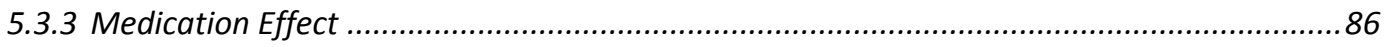

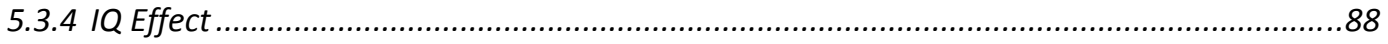

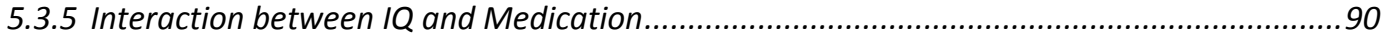

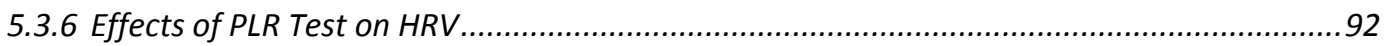

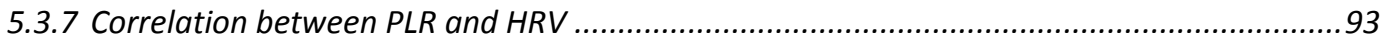

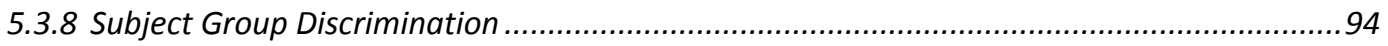

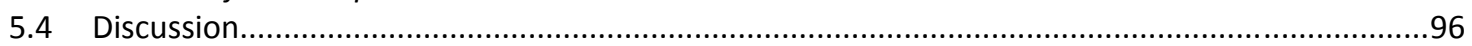

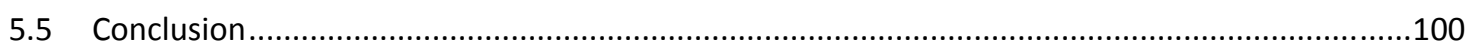

6 ASSOCIATION BETWEEN SENSORY PROCESSING AND PUPILLARY LIGHT REFLEX IN CHILDREN WITH AUTISM SPECTRUM DISORDERS .............................102

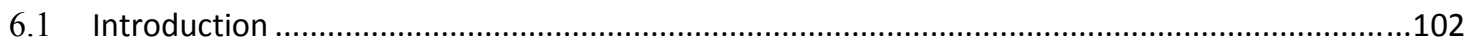

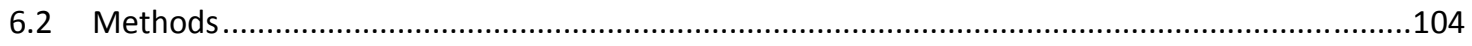

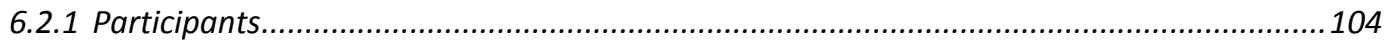

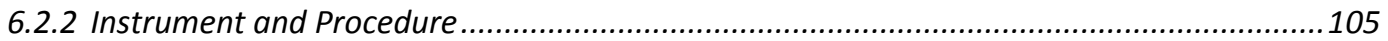

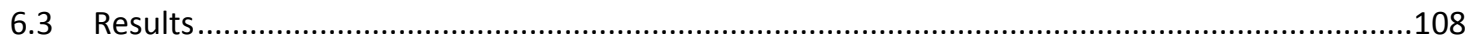

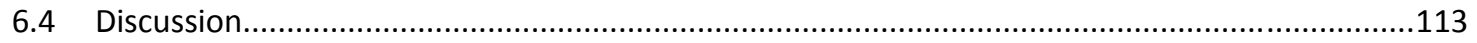

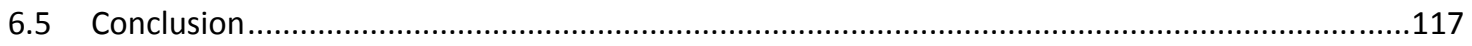

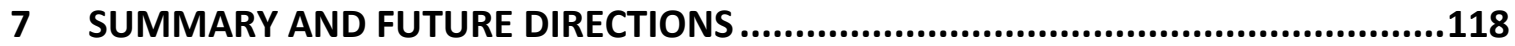

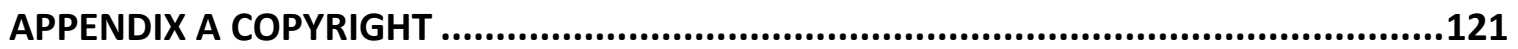

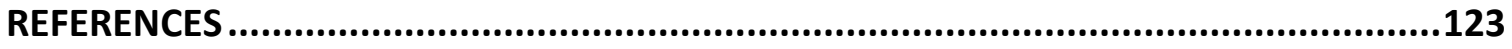

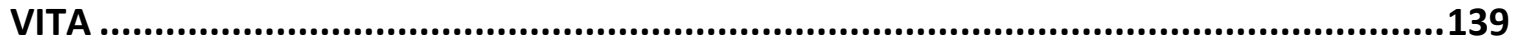




\section{LIST OF FIGURES}

$\begin{array}{ll}\text { Figure } & \text { Page }\end{array}$

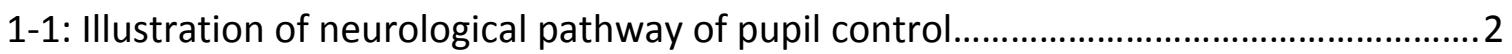

1-2: An illustration of the pupilogram and the associated PLR parameters........................ 9

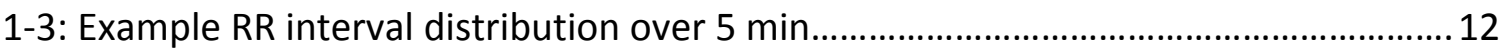

1-4: Power spectral density of RR tachogram shown in Fig. 1-3...................................... 19

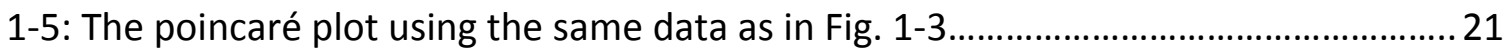

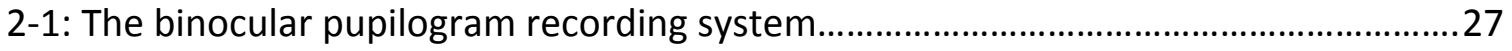

2-2: Average pixel value curve used to calibrate synchronization between stimulus

light pulse and image acquisition......................................................................... 29

2-3: An example to illustrate the pupil segmentation used in our study...........................30

2-4: An incomplete pupil image and resulting incorrect elliptical fitting...........................31

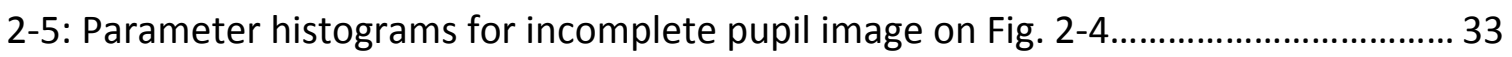

2-6: Correct fitting result produced by random Hough transform for the image shown in Fig. 2-4............................................................................................ 34

2-7: Pupilograms constructed using two methods for the same incomplete pupil image sequence....................................................................................................... 35

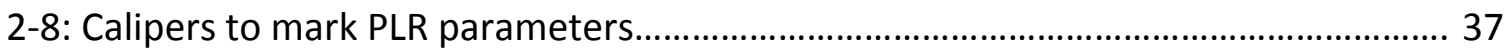

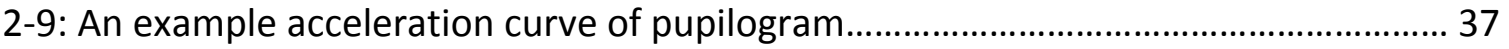

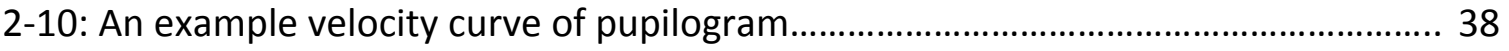

3-1: An illustration of the test procedure used in this study........................................... 45 
3-2: A comparison of frequency domain HRV parameters during different testing phases.

4-1: The age effect in resting pupil diameter in children with typical development...... 62

4-2: The age effect in PLR parameters obtained in children with typical development from 6 to 17 years old.

4-3: PLR parameters obtained in children with neurodevelopment disorders from 6 to 17 years old

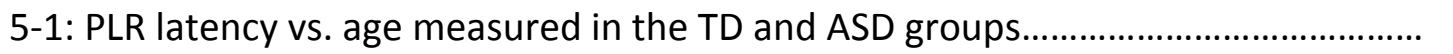

5-2: The age effect in average heart rate, SDNN and $\mathrm{HF}_{\mathrm{N}}$

5-3: Medication effect on PLR parameters.................................................................. 86

5-4: Medication effect on average heart rate and HRV parameters............................. 87

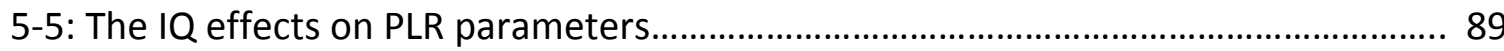

5-6: The IQ effects on average heart rate and HRV parameters...................................... 90

5-7: The effect of IQ and medication interaction on PLR latency.................................. 91

5-8: The change of frequency domain HRV parameters between consecutive HRV measurement phases.

5-9: The correlation between average heart rate and relative constricition amplitude.

5.10: The receiver-operating characteristic $(\mathrm{ROC})$ curve for the logistic regression model.

6-1: The correlation between PLR constriction amplitude and total sensory score. 109

6-2: PLR constriction amplitude at different scores for behavior items

6-3: The correlation between average heart rate and sensory total score. 


\section{LIST OF TABLES}

Table

1-1: Neurotransmitters/neuropeptides/second messengers found on parasympathetic pathway of pupil control

1-2: Neurotransmitters/neuropeptides/second messengers found on sympathetic pathway of pupil control.

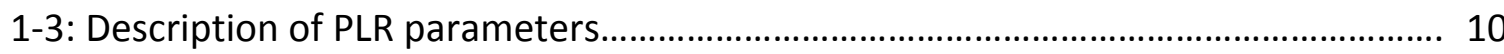

1-4: Description of HRV parameters calculated using time domain analysis................... 16

1-5: Description of HRV parameters calculated using frequency domain analysis........... 20

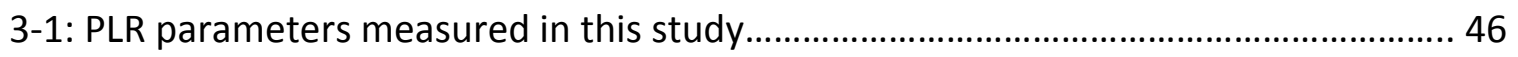

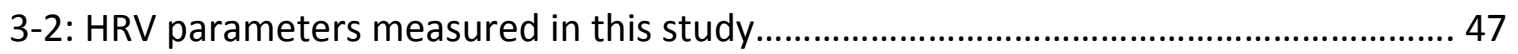

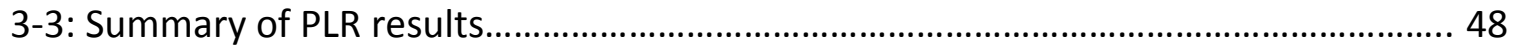

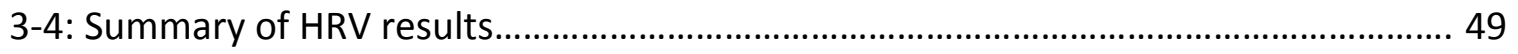

3-5: Correlations between average heart rate measured in different testing phases.... 50

3-6: Paired t-test results and corresponding effect sizes for comparison of frequency domain HRV parameters during different testing phases............................... 51

3-7: Factor loadings for the entire data set by using factor analysis.............................. 53

5-1: Distribution of IQ and medication use in TD, ASD and NDD groups.......................... 75

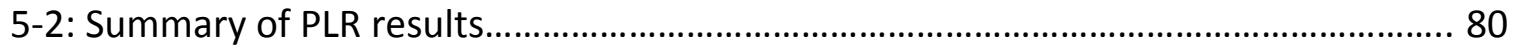

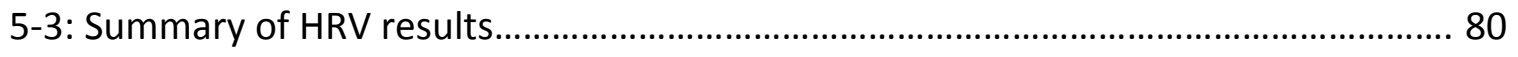


6-1: Spearman rank correlation coefficient between total sensory score and PLR parameters at each stimulus in TD and ASD groups...

6-2: Spearman rank correlation coefficient between total sensory score and PLR constriction amplitude at each stimulus in ASD sub-groups

6-3: Parameter estimates obtained from the PLS regression model and Post-hoc ANOVA analysis for constriction amplitude in the ASD group.

6-4: Spearman rank correlation $r$ for the correlation of PLR constriction amplitude with sensory score A and sensory score B in ASD group.................................... 113 


\section{LIST OF ACRONYMS}

Ach

ADHD

AHR

ANCOVA

ANOVA

ANS

ApEn

AR

ASD

AV node

cAMP

CCK

CD

CG

CGRP

DA

DFA

DFT

DLS fitting

DTI

DWI

ECG

EW nucleus

FA

FFT

fps

GAL

HF

$\mathrm{HF}_{\mathrm{N}}$

$\mathrm{HR}$

HRV

ipRGC

IQ

LA

LF
Acetylcholine

Attention-deficit/hyperactivity disorder

Average heart rate

Analysis of covariance

Analysis of variance

Autonomic nervous system

Approximate entropy

Auto regression

Autism spectrum disorders

Atrioventricular node

Cyclic adenosine monophosphate

Cholecystokinin

Correlation dimension

Ciliary Ganglion

Calcitonin gene-related peptide

Dark adapted

Detrended fluctuation analysis

Discrete Fourier transform

Direct least square fitting

Diffusion tensor imaging

Diffusion weight magnetic resonance imaging

Electrocardiogram

Edinger-Westphal nucleus

Fractional anisotropy

Fast Fourier transform

frames per second

Galanin

High frequency $(0.15-0.4 \mathrm{~Hz}$ )

High frequency normalized power

Heart rate

Heart rate variability

Intrinsically photosensitive retinal ganglion cells

Intelligence quotient

Light adapted

Low frequency $(0.04-0.15 \mathrm{~Hz})$ 


\begin{tabular}{|c|c|}
\hline LF/HF & Ratio between low frequency and high frequency power \\
\hline LLE & Largest lyapunov exponent \\
\hline MANOVA & Multivariate analysis of variance \\
\hline MRI & Magnetic resonance imaging \\
\hline ND & Neutral density \\
\hline NDD & Neurodevelopmental disorders \\
\hline NE & Noradrenaline \\
\hline NKA & Neurokinin A \\
\hline NN interval & $\begin{array}{l}\text { Normal-to-normal intervals (the time interval between consecutive } \\
\text { normal beats) }\end{array}$ \\
\hline NO & Nitric oxide \\
\hline NPY & Neuropeptide Y \\
\hline PACAPS & Pituitary adenylate cyclase peptides \\
\hline PDD-NOS & Pervasive developmental disorder-not otherwise specified \\
\hline PLR & Pupillary light reflex \\
\hline PLS regression & Partial least squares regression \\
\hline PNS & Parasympathetic nervous system \\
\hline PON & Pretectal olivary nucleus \\
\hline PSD & Power spectral density \\
\hline RAPD & Relative afferent pupillary defect \\
\hline RHT & Randomized Hough transform \\
\hline rMSSD & Root mean square successive difference of the RR intervals \\
\hline RPM & Ravens progressive matrices \\
\hline RR interval & $\begin{array}{l}\text { Interval from the peak of one QRS complex to the peak of the next on } \\
\text { electrocardiogram }\end{array}$ \\
\hline RSA & Respiratory sinus arrhythmia \\
\hline SA node & Sinoartial node \\
\hline SAS & Statistical analysis system \\
\hline SCG & Superior cervical ganglion \\
\hline SDNN & Standard deviation of normal-to-normal intervals \\
\hline SNS & Sympathetic nervous system \\
\hline SOM & Somatostatin \\
\hline SP & Substance P \\
\hline TD & Typically developing \\
\hline VEP & Visual evoked potentials \\
\hline VIP & Vasoactive intestinal polypeptide \\
\hline VLF & Very low frequency $(0-0.04 \mathrm{~Hz})$ \\
\hline
\end{tabular}




\title{
PUPILLARY LIGHT REFLEX IN CHILDREN WITH AUTISM SPECTRUM DISORDERS
}

\section{Chathuri Daluwatte}

\section{Dr. Gang Yao, Dissertation Supervisor}

\begin{abstract}
Pupillary light reflex (PLR) refers to the phenomenon of pupil size changing with respect to retinal illumination. It's a noninvasive, functional test which can reveal a rich set of information about nervous system. Abnormal PLR in children with autism spectrum disorders (ASD) was previously reported in a small population. In this research, a series of systematic studies were carried out to investigate the association of atypical PLR with ASD in a large population.

An experimental protocol was developed to measure PLR simultaneously with heart rate variability (HRV), a commonly used autonomic nervous system (ANS) measure. Our results indicate that variations of PLR and HRV are not associated in typically developing children. However, significant age effects on both PLR and HRV were observed in this population. In typically developing children, the resting pupil diameter increased with age significantly up to age 12. PLR constriction increased with age in children younger than 8 years old and reached a plateau thereafter. PLR latency decreased significantly from 6 to 9 years and stabilized thereafter. The average heart rate $(A H R)$ decreased with age in typically developing children. Standard deviation of normal-to-normal intervals (SDNN) showed little change before 12 years of age but was
\end{abstract}


increased in older children. High frequency normalized power $\left(\mathrm{HF}_{\mathrm{N}}\right)$ decreased with age in typically developing (TD) group.

PLR and HRV were also measured in 152 children with ASD and 36 children with non-ASD neurodevelopmental disorders (NDDs). The results showed atypical PLR in the ASD group including longer PLR latency, reduced relative constriction amplitude, and shorter constriction/redilation time. Similar atypical PLR parameters were observed in the NDD group. The ASD and NDD groups had faster AHR than the TD group. The NDD group also showed a significantly faster AHR than the ASD group. The age effect on PLR latency which was observed in typically developing children of 6-9 years old was not observed in the ASD and NDD groups. However, the age effect on HRV in the ASD group was similar to the age effect which was observed in the TD group. PLR constriction amplitude showed a significant negative correlation with average heart rate in children with ASD, but not in typically developing children.

ANS is also involved in regulating sensory behavior which is commonly atypical in children with ASD. We investigated potential association between PLR and sensory behaviors (behavioral observations) in children with ASD. The results indicated a weak but significant correlation between PLR constriction amplitude and sensory total score in the ASD group, but such correlation was not observed in typically developing children.

This study produced solid evidence that PLR pathway is affected in ASD. The reduced PLR constriction amplitude in children with ASD is associated with ANS dysfunction. Our results also suggest that PLR may be used as simple tool for monitoring developmental trajectory in children. 


\section{CHAPTER 1}

\section{INTRODUCTION}

\subsection{Pupillary Light Reflex}

\subsubsection{Introduction}

The pupil controls the amount of light entering the eye. Pupillary light reflex (PLR) describes the change of pupil size in response to the change of retinal illumination. However, the size of the pupil is also sensitive to many other stimuli apart from light intensity such as spatial structured patterns (Slooter and Van Norren, 1980; Ukai, 1985) and color stimuli (Young and Alpern, 1980; Young et al., 1993). Pupil response to light consists of two components: a steady state component and a transient component (Barbur, 2004). The steady state component is the response to ambient illumination (a step stimulus of light). The transient component is the response to a light flash (a short pulse stimulus of light).

\subsubsection{Neurological Pathway of PLR}

The pupil size is controlled by two iris muscles, sphincter and dilator, which are innervated by parasympathetic and sympathetic nervous systems, respectively. Parasympathetic and sympathetic nervous systems are subdivisions of the autonomic nervous system (ANS); the third subdivision is the enteric nervous system. Fig. 1-1 summarizes the neurological pathway of pupil control. 

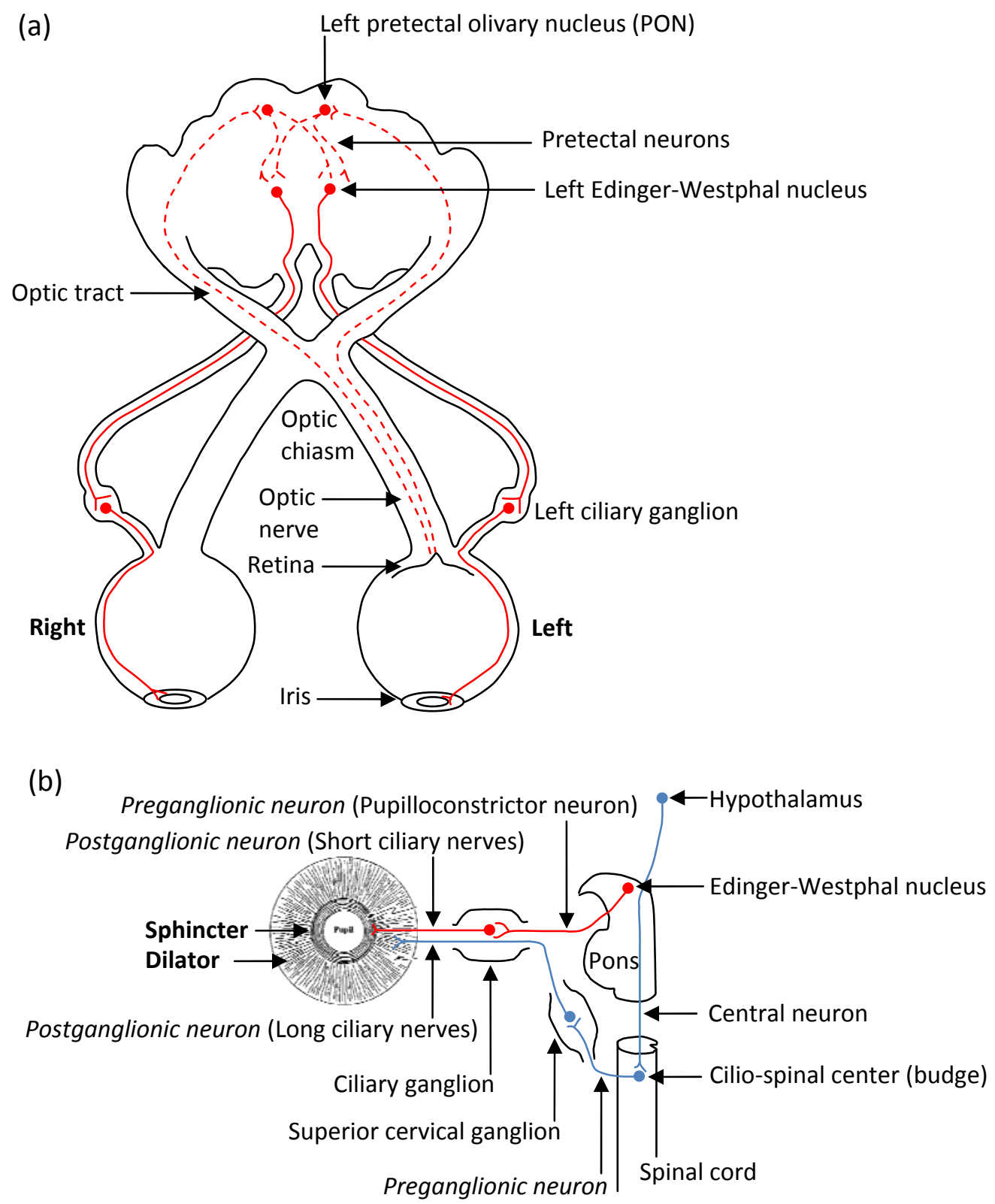

Fig. 1-1: Illustration of neurological pathway of pupil control.

(a) Afferent limb of PLR. (b) Efferent pathway of PLR.

Red dashed: Afferent pathway, Red: Parasympathetic pathway, Blue: Sympathetic pathway.

\subsubsection{Parasympathetic Pathway}

The afferent limb of PLR begins at the photoreceptors where both rods and cones contribute to the pupil response by integrating a light stimulus and transferring it 
to the retinal ganglion cells by way of the bipolar cells. About $1 \%$ of all retinal ganglion cells are devoted to the pupillary response, whereas the remaining cells project to the occipital cortex (via the lateral geniculate body) to subserve vision (Appenzeller, 1999). Apart from photoreceptors, intrinsically photosensitive retinal ganglion cells (ipRGCs) too mediate the pupillary response to light (Berson, 2007). The ipRGCs are less than 1$2 \%$ of the retinal ganglion cells and the photosensitivity of them are dependent on melanopsin (Panda et al., 2005). The response of these cells to dynamic light stimuli is sluggish, with a steep inverse correlation of latency with intensity. However, the steady state responses are stable, which is functionally different from that of rods and cones (Panda et al., 2005).

The retinal ganglion cell axons compose the optic nerves, and approximately $50 \%$ of the retinal ganglion cells from one eye decussate at the optic chiasm (Fig. 1-1a). Beyond the optic chiasm, with crossed and uncrossed fibers, optic nerves become optic tracts and because of the decussation, each optic tract contains homonymous visual and pupillary fibers.

The retinal ganglion cells responsible for pupillary action leave the optic tract and travel along the brachium of the superior colliculus to synapse in the pretectal olivary nucleus (PON) (Fig. 1-1a). After the signal is integrated in the PON, it is distributed to the right and left Edinger-Westphal (EW) nucleus equally (Fig. 1-1a). Thus, there is a double decussation of pupillary fibers at the optic chiasm and in the pretectal area. Therefore, unilateral light stimulation causes an equal direct and consensual pupillary constriction (Appenzeller, 1999). Constriction anisocoria refers to the 
phenomenon that direct pupillary constriction is slightly stronger than the consensual reaction. This may be due to asymmetry of crossing fibers at the chiasm or PON. Constriction anisocoria may be unilateral or bilateral but nevertheless the pupillary inequality is small and usually clinically insignificant (Appenzeller, 1999). The ipRGCs also project to the PON, contributing to the afferent limb of the pupillary light reflex (Kawasaki and Kardon, 2007).

The preganglionic neuron from EW nucleus synapses with the postganglionic neuron at the ciliary ganglion (CG) (Fig. 1-1b). This preganglionic neuron is included in the oculomotor nerve. There are three groups of nerve fibers in the CG: parasympathetic neurons, sympathetic (postganglionic) neurons, and sensory afferent neurons. The unmyelinated postganglionic parasympathetic neurons (short ciliary nerves) emerge from the CG and travel to the iris sphincter muscle (Appenzeller, 1999).

Throughout the parasympathetic pathway the synaptic transmission is mediated via neurotransmitter acetylcholine (Ach). Table 1-1 summarizes potential neuropeptides found in the parasympathetic pathway (Troger et al., 2007). Neuropeptides are a group of proteins which are distributed in the central and peripheral nervous system and act as neurotransmitters and/or neuromodulators. The distribution of the neuropeptides and their binding sites provide important information about neurological signaling pathways. 
Table 1-1: Neurotransmitters/neuropeptides/second messengers found on parasympathetic pathway of pupil control.

\begin{tabular}{lll}
\hline & Neuropeptide/Second messenger & Neurotransmitter \\
\hline \multirow{2}{*}{ Ciliary ganglion (CG) } & Choline acetyltransferase (Chat) & Acetylcholine (Ach) \\
& Tyrosine hydroxylase (TH) & Noradrenaline(NE), Adrenaline \\
\hline \multirow{2}{*}{ Short cilliary nerves } & Tyrosine hydroxylase (TH) & Noradrenaline(NE), Adrenaline \\
& Dopamine $\beta$ hydroxylase (DBH) & Noradrenaline(NE), Dopamine \\
\hline \multirow{5}{*}{ Sphincter } & Tachykinin NKA & \\
& Substance P & \\
& Neuropeptide Y (NPY) & \\
& Vasoactive intestinal polypeptide (VIP) \\
& Calcitonin gene-related peptide (CGRP) & \\
& Cholecystokinin (CCK) & \\
& Galanin (GAL) & \\
& somatostatin (SOM) & \\
& Pituitary adenylate cyclase peptides & \\
& (PACAPs) & \\
\hline
\end{tabular}

Fast contraction of sphincter muscle is found to be followed by a slow contraction which has been attributed to Ach and substance $\mathrm{P}$ (SP). The prominent presence of substance P- immunoreactive neurons (SP-IR) and corresponding binding sites in the sphincter muscle suggests a functional role of this peptide in the regulation of pupillary motility (Troger et al., 2007). However, SP effects on the sphincter muscle are subject to desensitization. Tachykinin neurokinin A (NKA) is also prominently present in the human sphincter muscle. Contrary to SP, NKA-evoked contractions develop more rapidly. Pharmacological application of selective receptor agonists and antagonists confirmed the predominant role of NKA in the mediation of noncholinergic nonadrenergic contraction of the sphincter muscle (Troger et al., 2007). In contrast to cholinergic contraction, the tachykininergic contraction is nitric oxide-insensitive.

In humans, the SP receptor is coupled to the adenylate cyclase and induces muscle relaxation. In the diabetic rat iris, elevated levels of SP have been connected with deficits in pupillary diameter regulation in diabetes mellitus (Troger et al., 2007). 
Among other peptides, calcitonin gene-related peptide (CGRP) and, more potently, adrenomedullin relax the iris sphincter muscle (Troger et al., 2007). Cholecystokinin (CCK) also contracts the sphincter muscle in humans (Troger et al., 2007). Both galanin (GAL) and somatostatin (SOM) attenuate the cholinergic component but have no effect on the tachykininergic slow response. On the other hand, pituitary adenylate cyclase peptides (PACAPs) (PACAP-27 and PACAP-38) enhanced the twitch response by increasing cholinergic transmission in the rabbit sphincter muscle without affecting the slow tachykininergic response (Troger et al., 2007).

\subsubsection{Sympathetic Pathway}

The sympathetic influence on the pupil consists of a three-neuron arc extending from the hypothalamus to the iris dilator muscle (Fig. 1-1b). The first order (central) neuron extends from the hypothalamus, descending ipsilaterally in the spinal cord to the ciliospinal center of Budge in the intermediolateral gray column on the spinal cord at C8-T1. The second order (preganglionic) neuron extends from the ciliospinal center of Budge to the superior cervical ganglion (SCG). Finally, the third order (postganglionic) neuron extends from the SCG to the iris dilator muscle (Appenzeller, 1999). These unmyelinated sympathetic fibers (postganglionic) originating in the SCG travel through the CG without synapsing (Fig. 1-1b) and enter through the superior orbital fissure associated with the nasociliary nerve to innervate the dilator muscle. In this three-

neuron arc, at the first two neuromuscular junctions, the synaptic transmission is mediated by neurotransmitter acetylcholine (Ach), while the postganglionic fibers 
synapse the dilator muscle by noradrenaline (NE). Neuropeptide evidence reported in the sympathetic pathway are summerizied in Table 1-2.

Table 1-2: Neurotransmitters/neuropeptides/second messengers found on sympathetic pathway of pupil control.

\begin{tabular}{lll}
\hline $\begin{array}{l}\text { Pre-ganglionic neuron (Superior } \\
\text { cervical ganglion) }\end{array}$ & Neuropeptide/Second messenger & Neurotransmitter \\
\hline & Choline acetyltransferase (Chat) & Acetylcholine (Ach) \\
& Glutamate \\
\hline \multirow{2}{*}{ Superior cervical ganglion (SCG) } & Dopamine $\beta$ hydroxylase (DBH) & $\begin{array}{l}\text { Noradrenaline(NE), } \\
\text { Adrenaline } \\
\end{array}$ \\
& Neuropeptide Y (NPY) $\rightarrow$ Nociception & Noradrenaline(NE), \\
& Vasoactive intestinal polypeptide (VIP) & Dopamine \\
\hline \multirow{2}{*}{ Long ciliary nerves } & Dopamine $\beta$ hydroxylase (DBH) & \\
\hline & & Noradrenaline(NE), \\
& Dopamine & Dopamine \\
\hline \multirow{2}{*}{ Dilator } & Neuropeptide Y (NPY) & Dopamine \\
& Vasoactive intestinal polypeptide (VIP) & \\
& Cyclic adenosine monophosphate (cAMP) & \\
& Pituitary adenylate cyclase peptides (PACAPs) & \\
\hline
\end{tabular}

However, according to Troger et al., (2007) effect of neuropeptides on the dilator muscle is less well explored. Neuropeptide $\mathrm{Y}$ (NPY) is known to have no direct effect but it enhances the phenylephrine-induced muscle contraction, possibly through inhibition of adenylate cyclase, since cyclic adenosine monophosphate (cAMP) is suggested to relax the muscle. In rats, no effect of CGRP was found on the dilator muscle, but in rabbits a relaxing effect was observed and cAMP mediates this effect. Similar to vasoactive intestinal polypeptide (VIP), pituitary adenylate cyclase peptides (PACAPs) induced relaxation of the rabbit dilator muscle (Troger et al., 2007). 


\subsubsection{Reciprocal Innervation to Both Sphincter and Dilator}

There is some evidence of reciprocal autonomic innervations to both dilator and sphincter muscles. For example, cholinergic activity results in pupillary constriction. Meanwhile, there may be simultaneous inhibition of the dilator muscle, therefore enhancing the constriction. The converse for pupillary dilation also holds true (Appenzeller, 1999). It has been found in rats and cats that somatic nerve stimulation of the dilator is mediated via the EW nucleus and CG. It is not via the sympathetic nerves, because the effect persists upon transaction of the cervical sympathetic trunk. The exact transmission mechanisms are not yet understood (Neuhuber and Schrödl, 2011).

\subsubsection{PLR Parameters}

For quantitative analysis of PLR, a pupilogram (Fig. 1-2) is obtained by extracting the pupil size from acquired images and displayed as pupil size change with time. The resting pupil diameter $D_{0}$ was calculated by averaging pupil diameters obtained for $1 \mathrm{~s}$ before stimulus onset. 


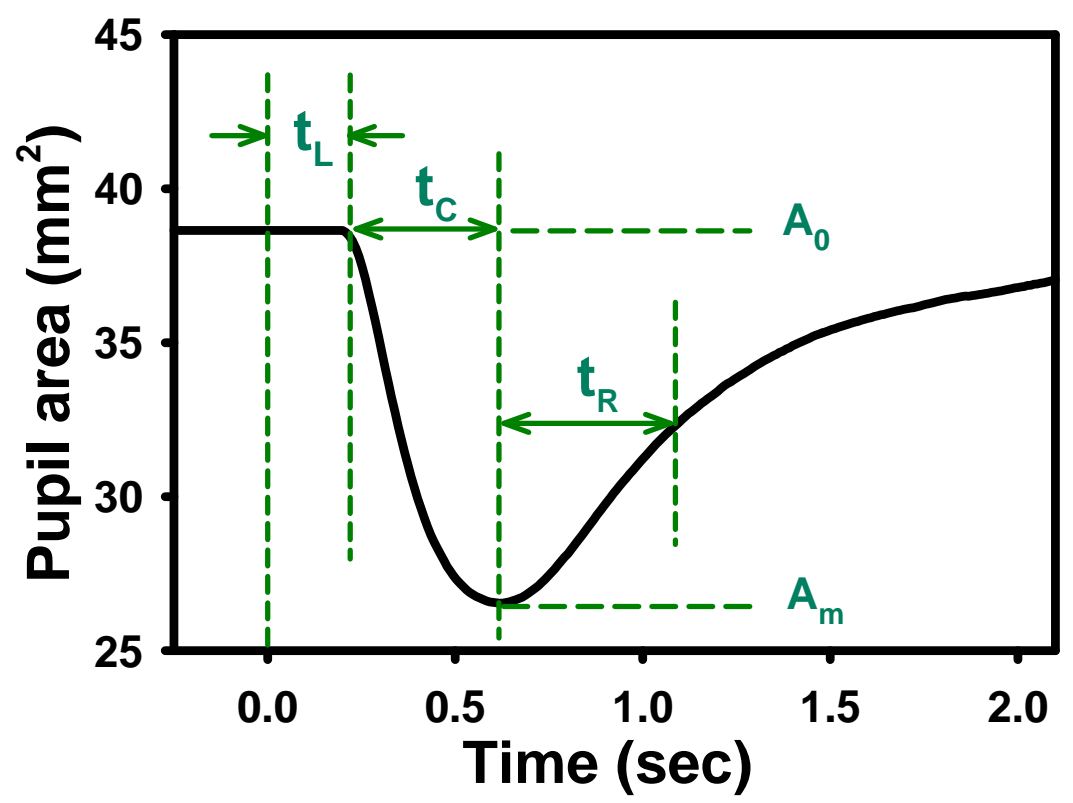

Fig. 1-2: An illustration of the pupilogram and the associated PLR parameters.

The optical stimulus is given at time zero. The baseline and minimal pupil diameters are calculated as $\mathrm{D}_{0}=2 \sqrt{\mathrm{A}_{0} / \pi}$ and $\mathrm{D}_{m}=2 \sqrt{\mathrm{A}_{\mathrm{m}} / \pi}$, respectively. The relative constriction amplitude is obtained as $A_{\%}=\left(A_{0}-A_{m}\right) / A_{0}$. The constriction and redilation velocities are calculated as $V_{c}=\left(D_{0}-D_{m}\right) / 2 \mathrm{t}_{C}$ and $V_{R}=\left(D_{0}-D_{m}\right) / 4 \mathrm{t}_{\mathrm{R}}$, respectively. $\mathrm{t}_{\mathrm{L}}$ : latency, $t_{C}$ :constriction time, $t_{R}$ : redilation time.

The PLR parameters explained in Table 1-3 were calculated from the pupilogram in Fig. 1-2 to quantify the pupillary response. Among these parameters the base pupil diameter, redilation time and redilation velocity are considered to be indicators of sympathetic modulation. Relative constriction amplitude, latency, constriction time and constriction velocity are considered to be indicators of parasympathetic modulation. Previous studies on PLR have suggested that PLR latency can be related to demyelination or atrophy in optical nerves (Alexandridis et al., 1981; Van Diemen et al., 1992). Animal studies have indicated the implication of cerebellum on PLR, and cerebellar lesions appear to be inducing larger PLR latencies and lesser high frequency 
responses compared to controls (Hultborn et al., 1978; Ijichi et al., 1977; Tsukahara et

al., 1973).

Table 1-3: Description of PLR parameters.

\begin{tabular}{|c|c|}
\hline Symbol (unit) & Definition \\
\hline $\mathrm{D}_{0}(\mathrm{~mm})$ & "Base pupil diameter": pupil diameter before stimulus onset \\
\hline $\mathrm{D}_{\min }(\mathrm{mm})$ & “Minimal pupil diameter": pupil diameter before stimulus onset \\
\hline$\Delta \mathrm{A}_{\%}$ & "Relative constriction amplitude": $\left(D_{0}^{2}-D_{\min }^{2}\right) / D_{0}^{2}$ \\
\hline$t_{L}(\mathrm{~ms})$ & $\begin{array}{l}\text { "Constriction latency": the elapsed time between light stimulus and beginning of } \\
\text { constriction }\end{array}$ \\
\hline$t_{c}(m s)$ & $\begin{array}{l}\text { "Constriction time": the time interval from beginning of constriction to the maximal } \\
\text { constriction }\end{array}$ \\
\hline$t_{R}(m s)$ & $\begin{array}{l}\text { "Redilation time": the time interval between maximal constriction and recovery to } \\
\text { half of the constriction }\left(D_{0}^{2}-D_{\min }^{2}\right) / 2 D_{0}^{2}\end{array}$ \\
\hline $\mathrm{v}_{\mathrm{c}}(\mathrm{mm} / \mathrm{s})$ & $\begin{array}{l}\text { "Constriction velocity": average velocity of the relative constriction }\left(D_{0}-D_{\min }\right) / 2 t_{c} \\
\text { "Recovery velocity": average velocity of the relative recovery }\left(D_{0}-D_{\min }\right) /\left(4 t_{p}\right)\end{array}$ \\
\hline
\end{tabular}

\subsubsection{Applications of PLR}

\subsubsection{Traditional Applications}

One of the traditional methods of pupillary assessment is to examine the size and shape of pupils in room light and dim light. Common examinations are assessment of the presence of aniscoria and the presence or absence of relative afferent pupillary defect (RAPD). RAPD can be assessed using the swinging-flashlight test. When the test is performed in an eye with an afferent pupillary defect, light directed in the affected eye will cause only mild constriction in both pupils, while light in the unaffected eye will cause a normal constriction in both pupils (due to an intact afferent path, and an intact consensual pupillary reflex). RAPD is an indication of a lesion of the optic nerve or severe retinal disease (Appenzeller, 1999). 
Horner's syndrome is a deficiency in occulosympathetic activity due to a lesion located along the three-neuron pathway of pupil dilation. This can be diagnosed by examining pupil dialtion using a cocaine drop test and/or a Paredrine test. Cocaine eyedrops block the reuptake of norepinephrine resulting in dilation of a normal pupil. In Horner's syndrome the lack of norepinephrine in the synaptic cleft causes dilation failure. The Paredrine test can locate the leison in the sympathetic pathway if it is in the third order neuron. If the third order neuron is intact, then the amphetamine causes neurotransmitter vesicle release which then releases norepinephrine into the synaptic cleft and resulting in robust dilation of the affected pupil. If the lesion itself is of the third order neuron, then the amphetamine will have no effect and the pupil remains constricted (Kaeser and Kawasaki, 2010).

\subsubsection{Marker of ANS Activity}

As an output of ANS activity, PLR provides a convenient and noninvasive tool for basic neuroscience research. Thus, PLR has been observed to study unusual ANS activity associated with various types of diseases such as AIDS (Maclean and Dhillon, 1993), depression (Fountoulakis et al., 1999; Sokolski and Demet, 1996), generalized anxiety

disorder (Bakes et al., 1990), Alzheimer's disease (Fotiou et al., 2000), mental retardation (Chaney et al., 1989), Down Syndrome (Sacks and Smith, 1989) and autism spectrum disorders (Fan et al., 2009a; Rubin, 1961). Peripheral neuropathies also provide clinical evidence of autonomic dysfunction. Pupil light reflex is promising in 
identifying autonomic neuropathy associated with diabetes (Smith and Smith, 1983;

Dütsch et al., 2004).

\subsection{Heart Rate Variability}

The pacemaker cells in the sinoartial (SA) node of the heart are capable of generating electrical impulses to cause coordinated and rhythmic contractions (Robertson, 2012). In addition to this intrinsic ability of the heart to regulate its electrical activity, ANS directly influences the conduction which introduces beat-to-beat variations in the heart rhythm. Heart rate variability (HRV) is the analysis of beat-to-beat variations and is considered to be a useful index in autonomic nervous system function evaluation (Malik et al., 1996). HRV is analyzed using the variations in the RR interval sequence extracted from the QRS wave obtained in electrocardiogram (ECG) (Fig. 1-3).

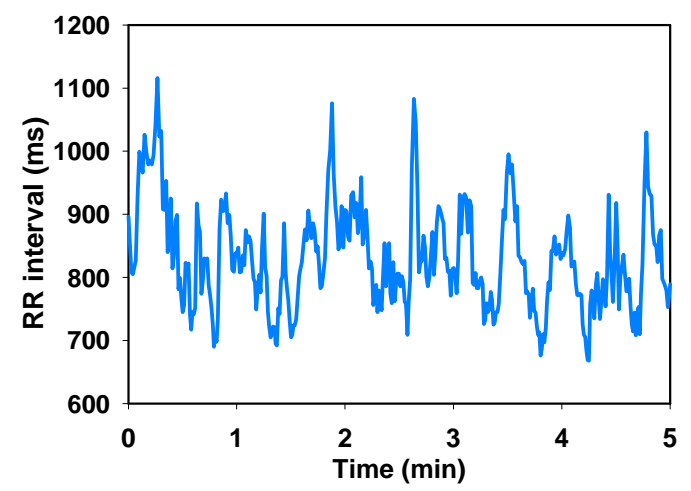

Fig. 1-3: Example RR interval distribution over $5 \mathrm{~min}$. 


\subsubsection{Neurological Pathway}

The functions of the heart are regulated by both divisions of the autonomic nervous system. The parasympathetic division inhibits and the sympathetic division facilitates cardiac activities. The central nervous system controls the two autonomic

divisions reciprocally. When parasympathetic activity increases, sympathetic activity decreases and vice versa. These effects are determined mainly by the relative abundance of nerve fibers, by the quantity of neurotransmitters and neuromodulators, and by the density of associated ligand receptors (Levy, 1997). However, at rest, parasympathetic control is predominant on the heart (Robertson, 2012), as illustrated by Katona et al., (1982) in their study of parasympathetic and sympathetic inhibitors' effects on heart rate.

\subsubsection{Parasympathetic Innervations}

The preganglionic cardiac vagal neurons are located in the medulla oblongata, in either the nucleus ambiguous or the dorsal nucleus of the vagus. After the preganglionic vagal fibers on each side exit from the skull, they travel down the neck in the carotid sheath. Efferent fibers from the two ANS divisions lie in separate nerve trunks. In the thorax, the efferent vagal fibers join the cardiac neural plexus, which is a complicated network of mixed nerve trunks. The individual nerves in the plexus contain preganglionic vagal and postganglionic sympathetic efferent fibers as well as many afferent nerve fibers from cardiopulmonary sensory receptors. The anatomical arrangement of the mixed nerves in the cardiac plexus differs considerably on the right and left sides. Each 
nerve bundle in the plexus is distributed to a distinct cardiac region, but the various regions overlap substantially. The preganglionic and postganglionic vagal neurons synapse in intracardiac ganglia or in epicardial fat pads, usually close to the structures innervated by the postganglionic neurons.

The pacemaker cells in the sinoartial (SA) node respond very rapidly to vagal activity because muscarinic receptors are coupled directly via guanine nucleotidebinding proteins (G proteins) to acetylcholine (Ach)-regulated potassium $\left(\mathrm{K}^{+}\right)$channels in the autonomic cell membranes; a slow secondary messenger system is not interposed. The prompt response enables beat-by-beat control of the cardiac rhythm. When vagal activity ceases, the response decays promptly. The Ach released into the cardiac tissues is quickly hydrolyzed because the enzyme acetyl cholinesterase is so abundant in the nodal regions of the heart (Levy, 1997).

\subsubsection{Sympathetic Innervations}

The cell bodies of the preganglionic sympathetic fibers to the heart are located in the intermediolateral columns of the lower two cervical and the upper eight thoracic segments of the spinal cord. These preganglionic fibers emerge through the white rami

communicantes and enter the paravertebral chain of ganglia. Most preganglionic cardiac sympathetic fibers ascend in the paravertebral chains and funnel through the stellate ganglia. The location of the synapses between the preganglionic and postganglionic cardiac sympathetic fibers in human are not established. 
The noradrenaline released from the sympathetic nerve endings increases the firing frequency of the autonomic cells in the sinoartial (SA) node due to a noradrenaline-induced increase in the slope of the slow diastolic depolarization in the automatic cells. After sympathetic neural activity to the heart ceases, the chronotropic response gradually decays back to the control level. The principal mechanisms that remove the neuronally released noradrenaline in the heart are: 1) reuptake by the sympathetic nerve endings and cardiac cells, and 2) diffusion away from the neuroeffector gap and into the coronary bloodstream (Levy, 1997). The characteristics of autonomic neural activity on the atrioventricular (AV) node are similar to those on automatic cells in the SA node. The vagal effects are inhibitory and the sympathetic effects are facilitatory (Levy, 1997).

\subsubsection{HRV Parameters}

To obtain HRV, the QRS waveform is measured using ECG. Using the QRS waveform, an RR tachogram (Fig. 1-3) is constructed. When constructing the RR tachogram for HRV analysis, all intervals between adjacent QRS complexes resulting from sinus node depolarizations, i.e. normal-to-normal (NN) intervals are used, while disregarding ectopic beats. RR tachogram is then analyzed using various methods to obtain heart rate variability. The techniques are divided into three categories: time domain, frequency domain and geometric methods (Malik et al., 1996). 


\subsubsection{Time Domain Analysis}

Time domain parameters are calculated using statistical analysis of the RR interval tachogram. Table 1-4 summarizes the parameters calculated using time domain analysis. Time domain parameters are most suitable in 24 hour measurements, as variance of parameters increases with the length of analyzed recording.

Table 1-4: Description of HRV parameters calculated using time domain analysis

\begin{tabular}{|c|c|}
\hline Symbol (unit) & Definition \\
\hline $\mathrm{AHR}(\mathrm{bpm})$ & Average heart rate \\
\hline SDNN (ms) & Standard deviation of normal NN intervals \\
\hline SDANN (ms) & $\begin{array}{l}\text { Standard deviation of the average NN intervals calculated over short periods, usually } 5 \\
\text { minutes }\end{array}$ \\
\hline SDNN index & The mean of the 5-minute standard deviations of NN intervals calculated over 24 hours \\
\hline rMSSD (ms) & Root mean square successive difference of the RR intervals \\
\hline NN50 (count) & number of succesive difference of intervals which differ by more than $50 \mathrm{~ms}$ \\
\hline pNN50 (\%) & $\begin{array}{l}\text { number of succesive difference of intervals which differ by more than } 50 \mathrm{~ms} \text { expressed as } \\
\text { a apercentage of the total no. of ECG cycles analyzed }\end{array}$ \\
\hline
\end{tabular}

SDNN, rMSSD, and pNN50 are calculated using following equations:

$$
\begin{aligned}
& \operatorname{SDNN}=\sqrt{\frac{1}{N-1} \sum_{j=1}^{N}\left(R_{j}-\overline{R R}\right)^{2}}, \\
& \text { MMSSD }=\sqrt{\frac{1}{N-1} \sum_{j=1}^{N-1}\left(R_{j+1}-R_{j}\right)^{2}}, \\
& \text { pNN50 }=\frac{N N 50}{N-1} \times 100 \%,
\end{aligned}
$$

where $\mathrm{RR}_{\mathrm{j}}$ is the $\mathrm{j}$ 'th $\mathrm{RR}$ interval; $\mathrm{N}$ is the total number of successive intervals; and $\overline{R R}$ is the mean value of RR intervals.

Since variance is mathematically equal to total power of spectral analysis, SDNN (Equation 1-1) reflects all the cyclic components responsible for variability in the period 
of recording. NN50 (Table 1-4), rMSSD (Equation 1-2), and pNN50 (Equation 1-3) represent short-term variation and estimate high-frequency variations in heart rate (Malik and Eckberg, 1998).

\subsubsection{Frequency Domain Analysis}

RR tachogram can be analyzed using spectral analysis, as power spectral density (PSD) provides basic information of how power (variance) distributes as a function of frequency. Various methods of calculating PSD are used: Fourier Transform, Auto Regression (AR) methods, Welch periodogram and Lomb periodogram.

Discrete Fourier transform (DFT) is widely used due to its simplicity of implementation. The $f(t)$ signal, is uniformly sampled at $1 / 2 f$ seconds. To compose set of uniform samples $\mathrm{f}(\mathrm{n} / 2 \mathrm{f})$, the Fourier spectrum is defined as Equation 1-4 (Yen, 1956).

$$
\begin{aligned}
& S(\omega)=\left\{\begin{array}{ll}
\frac{1}{2 \mathrm{f}} \sum_{\mathrm{n}=-\infty}^{\infty} \mathrm{f}(\mathrm{n} / 2 \mathrm{f}) \mathrm{e}^{-\mathrm{in} \omega / 2 \mathrm{f}}, & |\omega| \leq 2 \pi f \\
0 & ,|\omega|>2 \pi f
\end{array},\right. \\
& \mathrm{f}(\mathrm{t})=\sum_{\mathrm{m}=-\infty}^{\infty} \mathrm{f}\left(\tau_{\mathrm{m}}\right) \psi_{\mathrm{m}}(\mathrm{t}),
\end{aligned}
$$

where

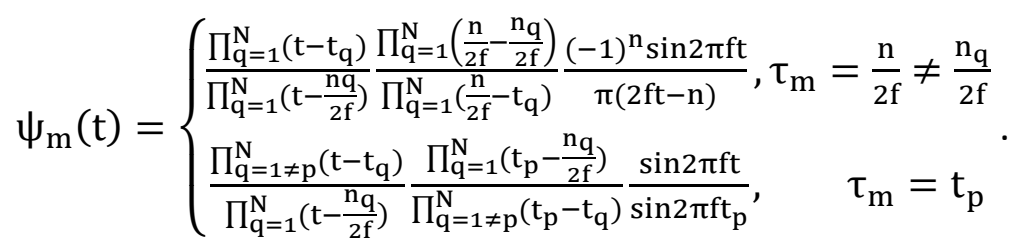

Because the RR tachogram is an unevenly sampled signal, the Fourier spectrum for the RR tachogram is calculated by treating it as a nonuniform sampled, bandwidth limited signal in Equations 1-4 and 1-5 (Yen, 1956). Using Equation 1-5, the nonuniform sample point distribution is migrated to a uniform sample point distribution. The sample 
point located at $t_{p}$ is migrated to a new position $t=n_{p} / 2 f$ where $2 \mathrm{ft}_{\mathrm{p}}$ is not an integer (Yen, 1956).

AR spectrum provides improved resolution for short-period samples, but the complexity of model order selection and contingency of negative components in spectral factorization are disadvantageous. An AR model predicts current values of a time series from past values of the same series, using the autocorrelation function. When $x[n]$ is the data sample with $N$ data points, the AR model is a set of autocorrelation functions and is defined as per Equation 1-7:

$$
x[n]=\sum_{i=1}^{M} a_{i} x[n-i]+\varepsilon[n],
$$

where $x[n]$ is the current value of the time series, $a_{1} \ldots a_{M}$ are predictor (weighting) coefficients, $M$ is the model order (number of past values used to predict the current value) and $\varepsilon[n]$ is the one-step prediction error (the difference between predicted value and current value). The first step of AR model identification is estimating predictor coefficients $\left(a_{1} \ldots a_{M}\right)$. This is done using least-squares minimization techniques to produce minimum error $\varepsilon[n]$ using the autocorrelation method (Yule-Walker equation). The AR model is then identified by determining model order (M). The most common criterion used to determine $\mathrm{M}$ is by minimizing the Akaike's Information Criterion (AIC) which is as defined in Equation 1-8:

$$
A I C(M)=N \ln \left(\sigma_{p}^{2}\right)+2 M
$$

where $\sigma_{p}^{2}$ is the prediction error variance. After the model is identified, its validity must be checked by confirming that the error terms are Gaussian distributed with a zero 
mean. Once predictor coefficients $\left(a_{1} \ldots a_{M}\right)$ and model order $(M)$ are determined the $A R$ spectrum is defined as Equation 1-9:

$$
R\left(e^{j \omega}\right)=\frac{\sigma_{p}^{2}}{\mid 1-a_{1} e^{-j \omega_{-} \cdots-\left.a_{M} e^{-j M \omega}\right|^{2}}}
$$

While Fourier transform and AR spectrum are the most common methods used to calculate power spectral density of RR tachogram, there are other techniques which address inherent caveats of the PSD calculation and improve performance. Welch periodogram is a DFT-based technique which decreases variance of the DFT spectrum by averaging the DFT spectrums of the overlapping segments of the whole HRV sample to obtain the final spectrum. Lomb periodogram is a least-squares frequency analysis technique for unequally spaced data and thus is robust at presence of missing beats (e.g.: ectopic beats in heart rate signal).

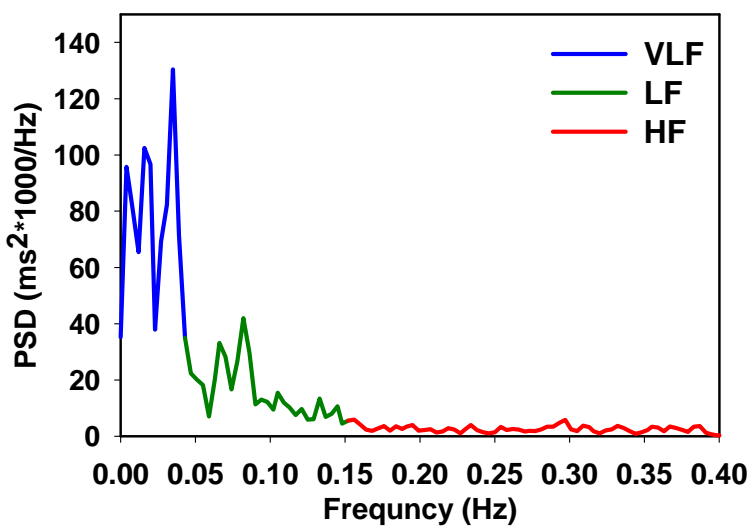

Fig. 1-4: Power spectral density of RR tachogram shown in Fig. 1-3.

Once the PSD (Fig. 1-4) is calculated using either of these algorithms, frequency domain parameters listed in Table 1-5 are calculated. While using frequency domain 
techniques, the problem of "stationarity" is frequently discussed with long-term recordings and hence frequency domain techniques are recommended to use in shortterm recordings (Malik and Eckberg, 1998).

Table 1-5: Description of HRV parameters calculated using frequency domain analysis.

\begin{tabular}{ll}
\hline Symbol (unit) & \multicolumn{1}{c}{ Definition } \\
\hline Absolute Power $\left(\mathrm{ms}^{2}\right)$ & Absolute powers in each frequency band $(\mathrm{VLF}, 0-0.04 \mathrm{~Hz}, \mathrm{LF}, 0.04-0.15 \mathrm{~Hz}, \mathrm{HF}$, \\
& $0.15-0.4 \mathrm{~Hz})$ \\
$\mathrm{HF}_{\mathrm{R}(\%)}$ & Relative Power of $\mathrm{HF}$ band (HF power /Total power) $\times 100 \%$ \\
$\mathrm{LF}_{\mathrm{R}(\%)}$ & Relative Power of LF band (LF power /Total power) $\times 100 \%$ \\
$\mathrm{HF}_{\mathrm{N}}(\%)$ & Normalized Power of $\mathrm{HF}$ band $(\mathrm{HF}, 0.15-0.4 \mathrm{~Hz})$, i.e., the relative powers of HF \\
& band when removing Very Low frequency band power from total power. HF \\
& power $/($ Total power $-\mathrm{VLF}$ power $) \times 100 \%$ \\
$\mathrm{LF}_{\mathrm{N}}(\%)$ & $100-\mathrm{HF}_{\mathrm{N}}(\%)$ \\
$\mathrm{LF} / \mathrm{HF}$ (n.u.) & The ratio of $\mathrm{LF} / \mathrm{HF}$ power \\
\hline
\end{tabular}

\subsubsection{Geometric Methods and Nonlinear Methods}

Geometrical methods have been used to derive measures of variability by considering RR intervals as sample density distributions and geometric patterns. There are many geometric methods which are explored using complicated analysis techniques such as Poincaré plots, approximate entropy, nonlinear methods of analysis (correlation dimension (CD), largest Lyapunov exponent (LLE), approximate entropy (ApEn), Hurst exponent, fractal dimension, a slope of detrended fluctuation analysis (DFA) and recurrence plots) (Acharya et al., 2006). The geometric methods need a reasonable number of normal-to-normal (NN) interval data which require longer recording times, hence are inappropriate to assess short term changes in HRV (Malik et al., 1996). 


\section{Poincaré Plot}

One commonly used geometric method is Poincaré plot. Poincaré plot is constructed by plotting $R R_{j+1}$ as a function of $R R_{j}$ and fitting an ellipse to the plot (Fig. 1-5). Using the fitted ellipse, the width (SD1) and length (SD2) are determined. SD1 describes short term variability while SD2 describes long term variability (Brennan et al., 2001).

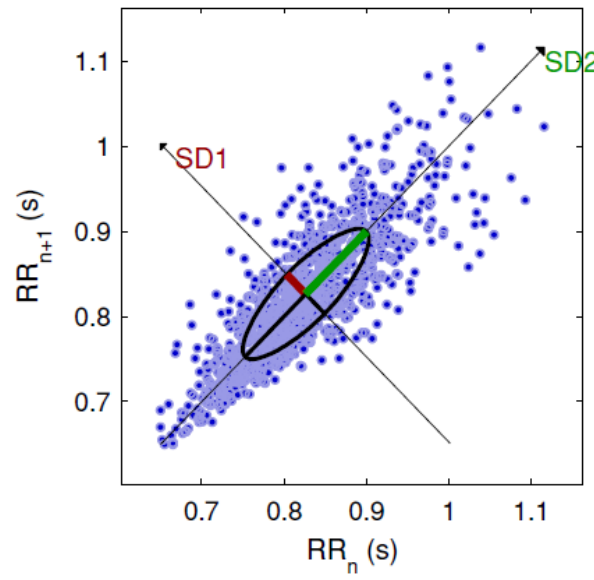

Fig. 1-5: The poincaré plot using the same data as in Fig. 1-3.

\section{Approximate Entropy}

Approximate entropy (ApEn) measures the irregularity of the signal and large values indicate high irregularity. To calculate ApEn, first a set of vectors are formed, when $\mathbf{m}$ is the embedding dimension, and $\mathrm{N}$ is the number of RR intervals:

$$
u_{j}=\left(R R_{j}, R R_{j+1}, \ldots, R R_{j+m-1}\right), j=1,2, \ldots, N-m+1 .
$$

Then the distance between vectors are calculated as follows:

$$
d\left(u_{j}, u_{k}\right)=\max \left\{\left|R R_{j+n}-R R_{k+n}\right| \mid n=0, \ldots, m-1\right\} .
$$


An index $C_{j}^{m}(r)$ is calculated to show the relative number of vectors $u_{k}$, which satisfy $d\left(u_{j}, u_{k}\right) \leq r$ for each $u_{j}$ with tolerance value $r$.

$$
C_{j}^{m}(r)=\frac{\text { number of }\left\{u_{k} \mid d\left(u_{j}, u_{k}\right) \leq r\right\}}{N-m+1}, \forall k .
$$

The approximate entropy is then calculated as:

$$
\operatorname{ApEn}(m, r, N)=\Phi^{m}(r)-\Phi^{m+1}(r),
$$

where

$$
\Phi^{m}(r)=\frac{1}{N-m+1} \sum_{j=1}^{N-m+1} \ln C_{j}^{m}(r) .
$$

The value of ApEn depends on $\mathrm{m}, r$ and N. A common choice for $r$ is 0.2 SDNN.

\section{Sample Entropy}

Sample entropy (SampEn) is similar to ApEn. However self-comparison with $u_{j}$ is excluded when calculating the index $C_{j}^{m}(r)$. Then, SampEn is calculated as Equation (115). Sample entropy is designed to reduce the bias ApEn has.

$$
\operatorname{SampEn}(m, r, N)=\ln \left(\frac{C^{m}(r)}{C^{m+1}(r)}\right),
$$

where

$$
C^{m}(r)=\frac{1}{N-m+1} \sum_{j=1}^{N-m+1} C_{j}^{m}(r) .
$$

\section{Detrended Fluctuation Analysis}

Detrended fluctuation analysis (DFA) is a measure of correlation within the signal (Peng et al., 1995). First the series $y(k)$ is derived as: 


$$
y(k)=\sum_{j=1}^{k}\left(R R_{j}-\overline{R R}\right), k=1, \ldots, N,
$$

where $\overline{R R}$ is the average RR interval. Then series $\mathrm{y}(\mathrm{k})$ is segmented into equal series of length $\mathbf{n}$ with a least square line fitted to each of these segments. If $y_{n}(k)$ are these regression lines, $F(n)$ is calculated over all segments as a function of $\mathrm{n}$ :

$$
F(n)=\sqrt{\frac{1}{N} \sum_{k=1}^{N}\left(y(k)-y_{n}(k)\right)^{2}} .
$$

Then $\alpha$ is calculated as the slope of the regression line relating $\log F(n)$ vs. $\log n$. Depending on the segment length $n$, the DFA correlations are divided into short-term and long-term fluctuations. The slope of $\log F(n)$ vs. $\log n$ for $4 \leq n \leq 16$ is defined as short-term fluctuations $\left(\alpha_{1}\right)$; whereas the slope for $16 \leq n \leq 64$ is defined as long-term fluctuations $\left(\alpha_{2}\right)$.

\subsubsection{Physiological Correlations and Reproducibility}

Heart rate and rhythm are largely under control of the ANS. Vagal tone and respiratory sinus arrhythmia (RSA) are found to be the principle contributors to the HF power of HRV (Malik et al., 1996; Berntson et al., 1997). Different interpretations exist for the LF component. Some studies (Malliani et al., 1991; Pagani et al., 1986) suggest that LF provides sympathetic activity, while other studies suggest that LF is affected by both sympathetic and parasympathetic activity (Malliani et al., 1994). The LF/HF ratio is considered by some investigators as an indication of sympathetic modulation; whereas others believe it to be of the sympathovagal balance (Eckberg, 1997). 
Several studies have shown that normalization to the total power is essential to avoid the effect of fluctuations in the total power and provides better reproducibility (Malik et al., 1996; Salo et al., 1999; Bernardi et al., 2011). La Fountaine et al. (2010) indicated that normalized units had the best intra-visit reliability relative to other HRV measures. Dupuy et al. (2012) also concluded that normalized HF power is the "most reliable parameter" in their study.

\subsubsection{Applications of HRV}

Although HRV was found to be associated with numerous cardiological and noncardiological diseases, a general consensus of its practical use in adult medicine has been reached only in two clinical scenarios: as a predictor in risk after acute myocardial infarction and as an early warning sign in diabetic neuropathy (Malik et al., 1996).

HRV has been identified as a predictor in risk after acute myocardial infarction. The depressed HRV (where SDNN values are below 50 and the HRV triangular index is less than 20 from 24-hour recordings) serves as a powerful predictor of mortality and arrhythmic complications (Malik et al., 1996; Lombardi et al., 2001; Huikuri et al., 2000). HRV is also useful in detecting diabetic autonomic neuropathy where short-term HRV was reported to have reduced power in all spectral bands, fail to increase LF on standing, and have abnormally reduced total power with unchanged LF/HF ratio; longterm HRV had less than 500 or abnormally low counts of NN50 on 24-hour HRV recordings (Braune and Geisendörfer, 1995; Pagani, 2000). HRV has also been found to correlate with mental and physical stress, and attention (Berntson et al., 1997). 


\subsection{Summary of this Dissertation Study}

The overall objective of this study is to investigate the pupillary light reflex in children with autism spectrum disorders.

To facilitate data processing in a large population, the existing custom PLR software was improved to reduce image processing time using multithreading and incorporate automatic calculation of PLR parameters. The graphic user interface (GUI) was also improved such that personnel without special skills could easily operate the software. In addition, a pupil segmentation algorithm based on randomized Hough transform was implemented to process incomplete pupil images due to half-opened eyes.

We tested PLR in children with ASD, typical development, and other non-ASD neurodevelopment disorders to study group differences in PLR. Age trends and effects of autism specific factors (intelligent quotient, autism diagnosis, medication used) on PLR were analyzed. The potential of using PLR as a screening tool for autism was explored.

To investigate the potential involvement of ANS dysfunction in atypical PLR, we studied the association between simultaneously measured PLR and heart rate variability. Both heart rate variability and pupillary light reflex have innervations from ANS (Barbur, 2004; Malik et al., 1996; Levy, 1997; Appenzeller, 1999). In fact, correlations between HRV and PLR parameters were previously reported in adults during exercise (Kaltsatou et al., 2011) and in patients with acute schizophrenia (Bär et al., 2008). However, Bär et al. (2009) found limited correlation between specific PLR and 
HRV parameters in healthy adults $19-64$ years old. An examination of these simultaneously obtained ANS measures can help identify neurophysiologic implications of both the systems.

In addition to PLR and HRV, ANS is involved in regulating a multitude of physiological and behavioral activities. Atypical behaviors such as sensory processing have been commonly reported in children with ASD. We hypothesized that behavioral measures and physiological measures such as PLR might be correlated due to the involvement of ANS. To test this hypothesis, we investigated the association between PLR parameters and sensory behaviors in children with ASD. 


\section{CHAPTER 2}

\section{INSTRUMENTATION*}

\subsection{Binocular Pupilogram Recording System}

A custom-built binocular pupilogram recording system (Fig. 2-1) was used to measure PLR in all our participants. The two recording channels are independent but synchronized with high spatial $(35 \mu \mathrm{m} /$ pixel) and temporal resolution $(8.7 \mathrm{~ms})$. The optical stimulation and image acquisition were controlled through a computer interface via a custom-developed Labview program. This customized system has two "sighting" ports so that the participant can fix sight at a given target during a PLR test. In addition, this system is versatile for setting various stimulation waveforms and intensities.
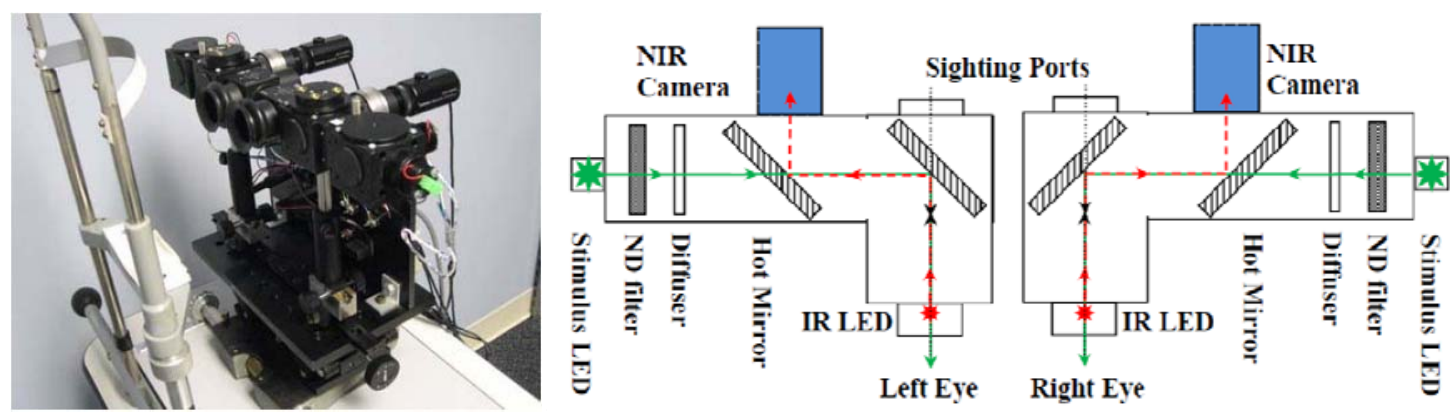

Fig. 2-1: The binocular pupilogram recording system.

(a) Picture of the binocular pupilogram recording system. (b) A schematic diagram of the binocular pupilogram recording system. A hot mirror was used in each channel to separate the optical stimulation path and imaging path. The participant can fix the sight on a monitor through the two sighting ports.

\footnotetext{
* Part of the material presented in this chapter was published in IEEE Xplore: Daluwatte, C., Miles, J. H., Christ, S. E., Beversdorf, D. Q., Lofgreen, A., Berliner, N. \& Yao, G., Age-dependent pupillary light reflex parameters in children. In: Engineering in Medicine and Biology Society (EMBC), 2012 Annual International Conference of the IEEE, 2012 San Diego, CA. 3776-3779.
} 
Pupils were illuminated by near infrared (NIR) LEDs at $880 \mathrm{~nm}$ wavelength. A 530 $\mathrm{nm}$ green LED was used to provide the $100 \mathrm{~ms}$ light stimulus for evoking the PLR. The optical stimulus illuminated a circular optical diffuser positioned at $12.5 \mathrm{~cm}$ from the eye with an effective diameter of $1.27 \mathrm{~cm}$ (an equivalent visual field of $5.7^{\circ}$ ). The electric current to the LED was controlled to vary the stimulation irradiance along with the use of neutral density (ND) filters. The stimulation intensities used in this study varied from $69.3 \mathrm{~cd} / \mathrm{m}^{2}$ to $8721.1 \mathrm{~cd} / \mathrm{m}^{2}$ in light-adaptation (LA) and was $63.1 \mathrm{~cd} / \mathrm{m}^{2}$ in darkadaptation (DA).

Two near infrared (NIR) cameras (GC660, Allied Vision Technologies, Stadtroda, Germany) were used in the system to acquire pupil images. The image size was 659 pixels $\times 494$ pixels with a 12 bit resolution. At each PLR test, the cameras were triggered first to acquire baseline pupil images for $1 \mathrm{~s}$. Then the green LEDs were triggered to give a 100ms flash. Image acquisition was continued for four more seconds to capture the entire pupil constriction and recovery process. A total of 575 images were acquired from each eye in a single test trial $(5 \mathrm{sec}$ ). All acquired images were saved using the tiff format.

\subsection{Calibration}

Calibrations were performed biweekly throughout the study duration (140 weeks) in order to obtain consistent results pertaining to power in stimulus LEDs, spatial resolution of camera and synchronization between light pulse and image acquisition. The light power was calibrated using an optical power meter (1830-C, Newport 
Corporation, United States). For both, left and right, channels the calibrated power was maintained within $\pm 10 \%$ of the reported nominal power values: $0.09 \mu \mathrm{W} / \mathrm{cm}^{2}, 1.0$ $\mu \mathrm{W} / \mathrm{cm}^{2}, 9.9 \mu \mathrm{W} / \mathrm{cm}^{2}$.

The spatial resolution of both channels were calibrated using a Pocket USAT optical test pattern target (1951 USAF, Edmund Optics, United States) at the same time as when LED power calibrations were performed. The image processing software was updated with corresponding spatial resolution values.

The synchronization between light pulse and image acquisition was calibrated by imaging a white sheet and calculating the average pixel value for each image. Starting from the $115^{\text {th }}$ image, approximately 11 images have a comparatively higher average pixel value, due to the flash of light (Fig. 2-2). Since the frame rate of the camera is $115 \mathrm{fps}$, this converts to a $1 \mathrm{~s}$ delay and $\sim 96 \mathrm{~ms}$ flash duration. Synchronization calibration was performed for both channels separately before every subject measurement.

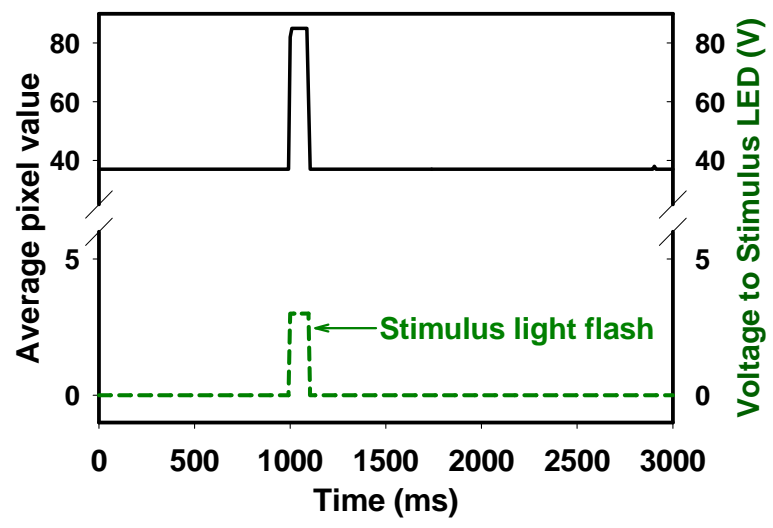

Fig. 2-2: Average pixel value curve used to calibrate synchronization between stimulus light pulse and image acquisition. 


\subsection{Image Processing}

Custom image processing software developed in visual $\mathrm{C}++$ was used to automatically calculate the pupil diameter from each of the recorded pupil images in the image sequence (575 images for each eye).

A histogram-based thresholding was applied after contrast stretching the pupil image to locate the pupil's boundary pixels. The threshold of pupil boundary was identified as the pixel value corresponding to the first minima of the image histogram as shown in Fig. 2-3b. Using this threshold the images were binarized and the pupil was segmented. All pixels on the pupil boundary were then extracted.

Two options were implemented to obtain an ellipse fitting to the segmented pupil boundary (Fig. 2-3a). In the first option, a direct least square fitting algorithm (Fitzgibbon et al., 1999) was applied. The area of the fitted ellipse was used to estimate the pupil area. A nominal diameter was calculated by treating the pupil as a circle.
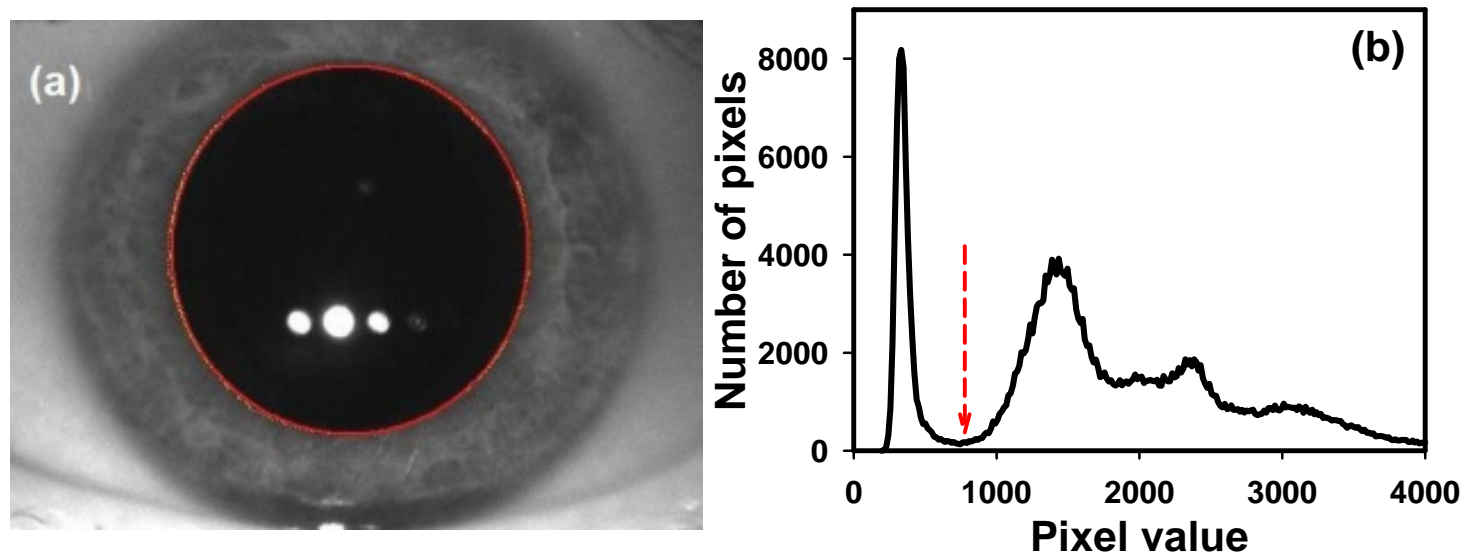

Fig. 2-3: An example to illustrate the pupil segmentation used in our study. (a) An example pupil image. (b)The corresponding histogram. The first minimum marked by the arrow in (b) indicates the boundary of the black pupil in (a). This value was used as the threshold to segment the pupil. The red circle in (a) shows the fitted ellipse using least square fitting. 
Incomplete pupils are often encountered in pupillometry, due to blinks or half opened eyes (Fig. 2-4). Such incomplete pupil images only contain a part of the pupil boundary ellipse and secondly, it also contains noisy elliptical curves introduced by the eyelid.

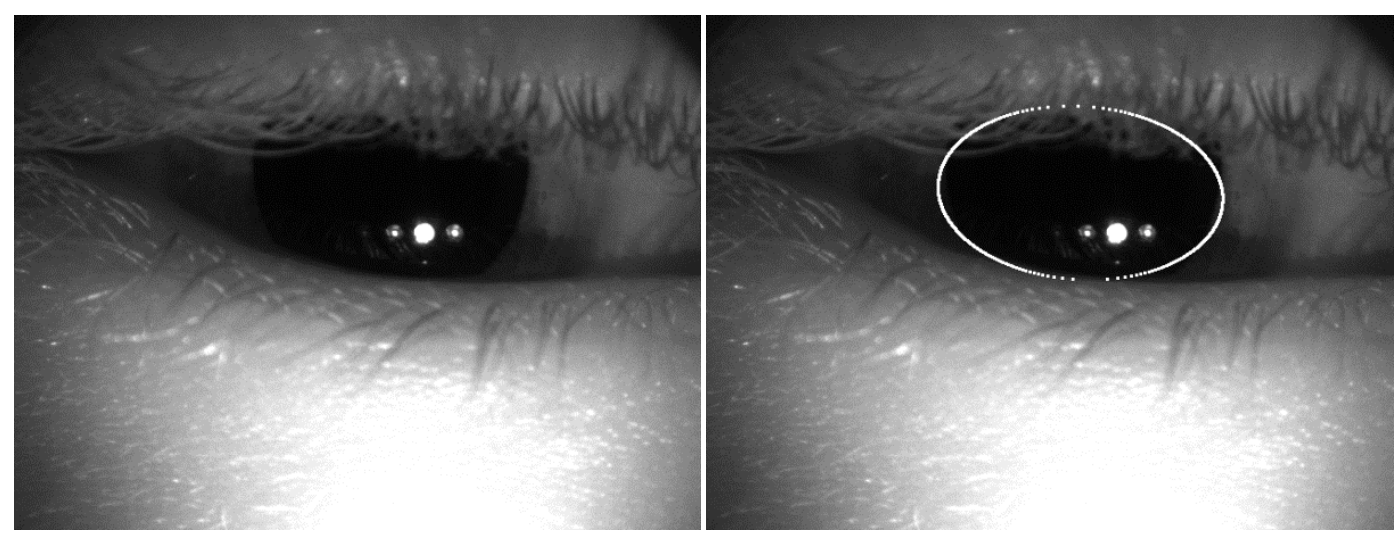

Fig. 2-4: An incomplete pupil image and resulting incorrect elliptical fitting. (a) An incomplete pupil image. (b) Incorrect fitting result produced by direct least square fitting algorithm due to noisy edges caused by eyelids.

In our test, we found pupillary images from $1 \%$ of the subjects in the typical control group, $6 \%$ in the ASD group and $13 \%$ of the children in the group with other neurodevelopmental disorders (NDD) could not be correctly processed using the above procedure due to eye movement and half opened eyes during the test.

An alternative option was implemented based on randomized Hough transform to fit cases where the direct ellipse fitting failed. The Hough transform estimates parameters by mapping the image space to a parameter space and detecting collinear points in the parameter space. Hough transform is robust against outliers and curve discontinuities in the image space. However the generalized implementation of Hough transformation is computationally expensive since the algorithm takes every combination of edge points into account before converging to the solution. Thus, 
different kinds of approaches have been adapted to reduce the computational cost involved. Randomized Hough transform uses a large enough random sample from all the combinations before converging to the solution to reduce the computational cost (Xu et al., 1990).

A parametrical linear model of an ellipse is:

$$
x^{2}+y^{2}-U\left(x^{2}-y^{2}\right)-V 2 x y-R x-S y-T=0,
$$

where elliptical parameters can be represented using a vector $p^{\prime}=\left[\begin{array}{lllll}U & V & R & S & T\end{array}\right]$. An iterative algorithm was used to calculate the ellipse parameters using 5 randomly selected points each time. At each iteration, randomly selected five pixels are used to solve the $\mathbf{A p}=\mathbf{k}$ system to estimate $\mathbf{p}$ where $A$ and $\mathbf{k}$ are:

$$
\begin{aligned}
A & =\left[\begin{array}{lllll}
\left(x_{i}^{2}-y_{i}^{2}\right) & 2 x_{i} y_{i} & x_{i} & y_{i} & 1
\end{array}\right]_{i \times 5} \quad i=1, \ldots, 5, \\
k & =\left[\begin{array}{lll}
x_{i}^{2} & y_{i}^{2}
\end{array}\right]_{i \times 2} \quad i=1, \ldots, 5 .
\end{aligned}
$$

Using the estimated Cartesian equation parameters, $p^{\prime}=$ $\left[\begin{array}{lllll}U & V & R & S & T\end{array}\right]$ ellipse center coordinates $\left(\mathbf{x}_{0}, \mathbf{y}_{0}\right)$ are first calculated as shown in Equation 2-4:

$$
\left[\begin{array}{l}
x_{0} \\
y_{0}
\end{array}\right]=\left[\begin{array}{cc}
2(1-U) & -2 V \\
-2 V & 2(1+U)
\end{array}\right]^{-1}\left[\begin{array}{l}
R \\
S
\end{array}\right]
$$

The angle $(\boldsymbol{\alpha})$ is then calculated as shown in 2-5:

$$
\alpha=\frac{1}{2} \tan ^{-1} V / U
$$

Using calculated $\mathbf{x}_{\mathbf{0}}, \mathbf{y}_{\mathbf{0}}$, and $\boldsymbol{\alpha}$, semi major axis (a) and semi minor axis (b) are calculated as shown in Equations 2-6 and 2-7:

$$
a=\sqrt{\frac{-\sin 2 \alpha}{(V-\sin 2 \alpha)}\left(T+\frac{x_{0} R}{2}+\frac{y_{0} S}{2}\right)},
$$




$$
b=\sqrt{\frac{\sin 2 \alpha}{(V+\sin 2 \alpha)}\left(T+\frac{x_{0} R}{2}+\frac{y_{0} S}{2}\right)} .
$$

To ensure that only ellipses representing the pupil are selected, the following conditions need to be met: $x_{0}, y_{0}, a$, and $b$ are all positive; $x_{0}$ and $y_{0}$ are not larger than the image width and height. An accumulator stores the detected ellipses which are candid ellipses of the pupil. Thus, if $0<x_{0}<$ width and $0<y_{0}<$ height and $a>0$ and $b>0$, add the ellipse to the accumulator.

Fig. 2-5 shows parameter histograms for the image shown in Fig. 2-4. The location of the highest peak in the histogram gives the best estimation for each parameter. The final solution for each parameter is the value corresponding to the peak on the histogram constructed using accumulated values.
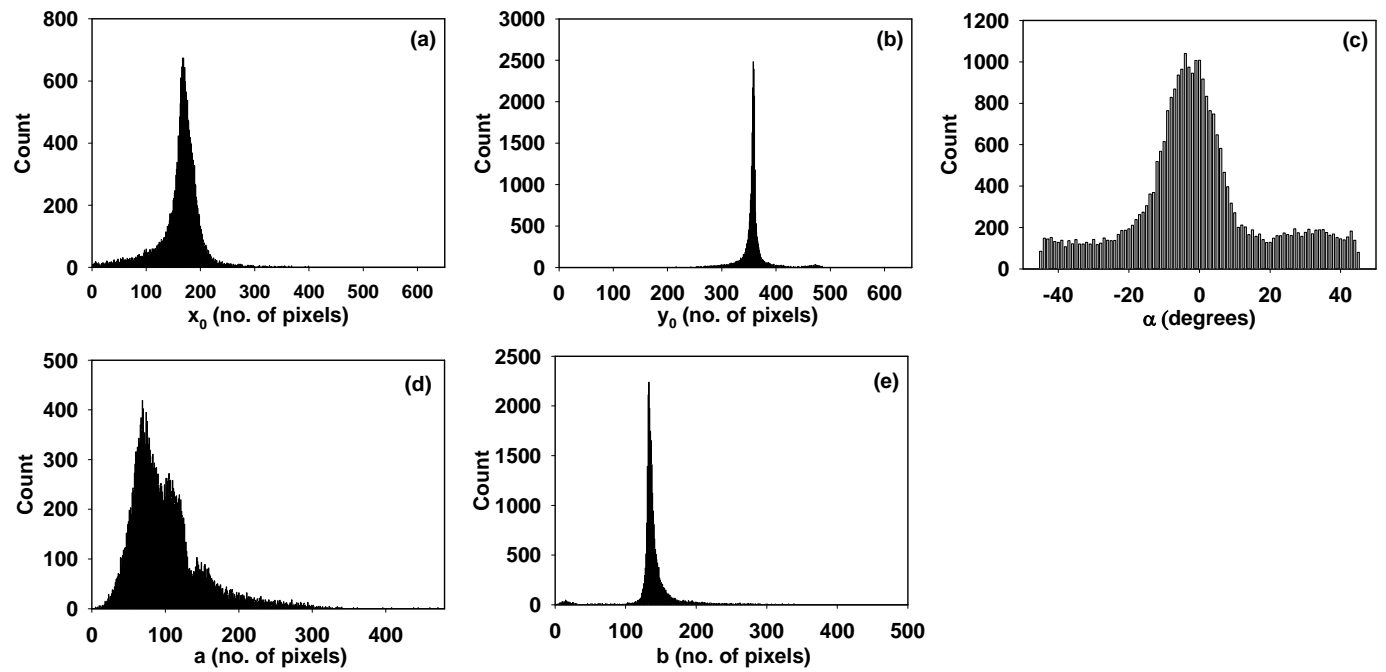

Fig. 2-5: Parameter histograms for incomplete pupil image on Fig. 2-4.

(a)Histogram for $x_{0}$. (b) Histogram for $y_{0}$. (c) Histogram for angle. (d) Histogram for semi major axis - a. (e) Histogram for semi minor axis - b. 

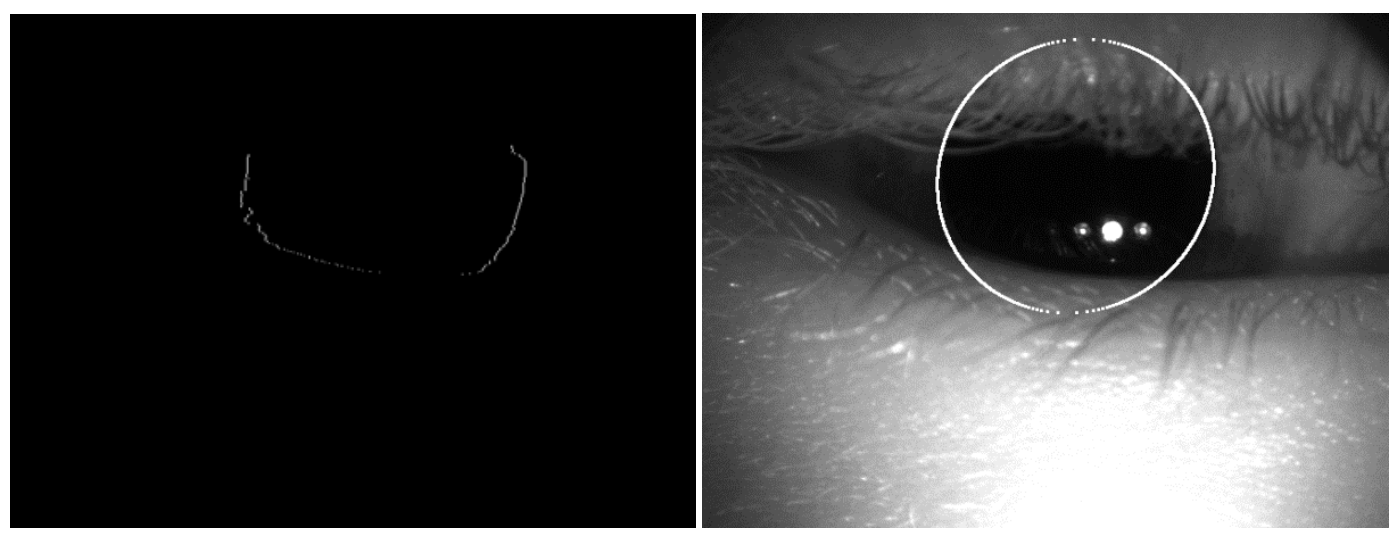

Fig. 2-6: Correct fitting result produced by random Hough transform for the image shown in Fig. 2-4.

(a) Segmented edge image. (b) Correct fitting result produced by random Hough transform.

Fig. 2-6 shows the correct ellipse fit obtained using random Hough transform for the same incomplete pupil images from Fig. 2-4. Fig. 2-7 provides a graphic rendition of pupilogram curves derived from the two methods. Also, since this algorithm showed the ability to automatically identify the correct ellipse, automatic processing was applicable for those instances which were previously resolved by manual intervention by deriving a region of interest. In order to measure the accuracy of the implemented random Hough transform algorithm, results obtained for complete pupil image sequences using random Hough transform $(\mathrm{RHT})$ were compared with direct least square (DLS) fitting, by treating direct least square fittings as the gold standard (Equation 2-8):

$$
\text { Error }=\frac{\text { (value } \left._{D L S}-\text { value }_{R H T}\right)}{\text { value }_{D L S}} \times 100 \%
$$

The calculated percentage error was always less than $6.0 \%$ for the pupil area. For parameters, the maximum percentage error is $<0 \%$ for latency, $<3 \%$ for constriction and 
redilation times, $<2 \%$ for base radius and minimal radius and $<1 \%$ for constriction amplitude.

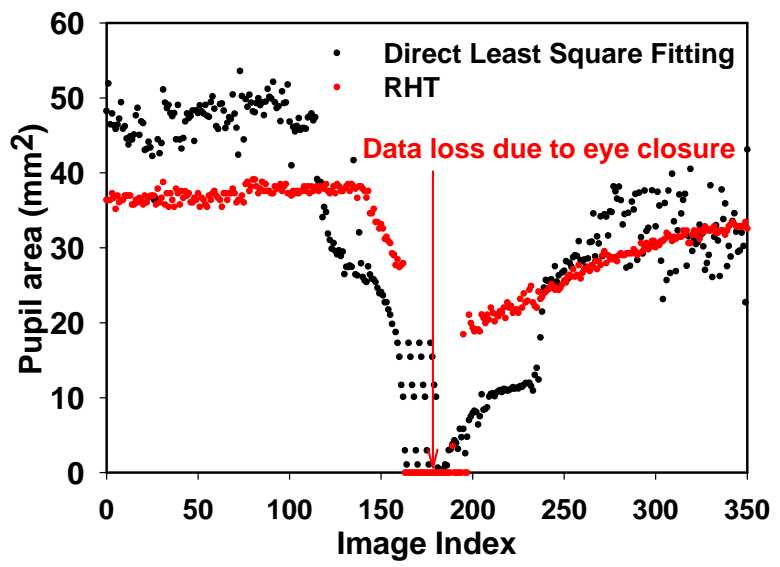

Fig. 2-7: Pupilograms constructed using two methods for the same incomplete pupil image sequence.

\subsection{Extracting PLR Parameters}

Once all pupil diameters were extracted from the acquired image sequence, a pupilogram curve (Fig. 1-2) was constructed to represent the pupil size change in response to the optical stimulus. The pupilogram was normalized against the resting pupil area to remove effects of resting pupil size when calculating constriction amplitude. The following PLR parameters were calculated from the pupilogram in Fig. 1-2 to quantify the pupillary response. The resting pupil diameter $D_{0}$ was calculated by averaging pupil diameters obtained during the $1 \mathrm{~s}$ period before stimulus onset. The relative constriction amplitude was calculated by normalizing the difference between the resting pupil area and the minimum pupil area against the resting pupil area. PLR latency $\left(t_{L}\right)$ was calculated as the time interval between stimulus onset and the 
beginning of pupil constriction. The constriction time $\left(t_{c}\right)$ was calculated as the time interval between the beginning of pupil constriction and when pupil reached minimal size. The redilation time $\left(t_{R}\right)$ was calculated as the time interval between the minimal pupil diameter and when the pupil recovered to half of the constriction. The pupilogram (before normalization) was smoothed by using a 6th order Savitzky-Golay filter. To measure the PLR latency, the acceleration (2nd order derivative) of the pupilogram was calculated. The time of the maximal acceleration was first identified and used as the starting point to back track toward the stimulation onset. The first image frame that deviated from the baseline pupil size was considered as the onset of pupil constriction.

Several GUI components of the image processing software were developed to facilitate extraction of the PLR parameters. First, calipers were implemented to mark PLR parameters: resting pupil size, minimum pupil size, latency, constriction time and redilation time (Fig. 2-8). These calipers can be changed manually (by dragging and dropping) and the corresponding value will be updated in the results file which saves the final result. 


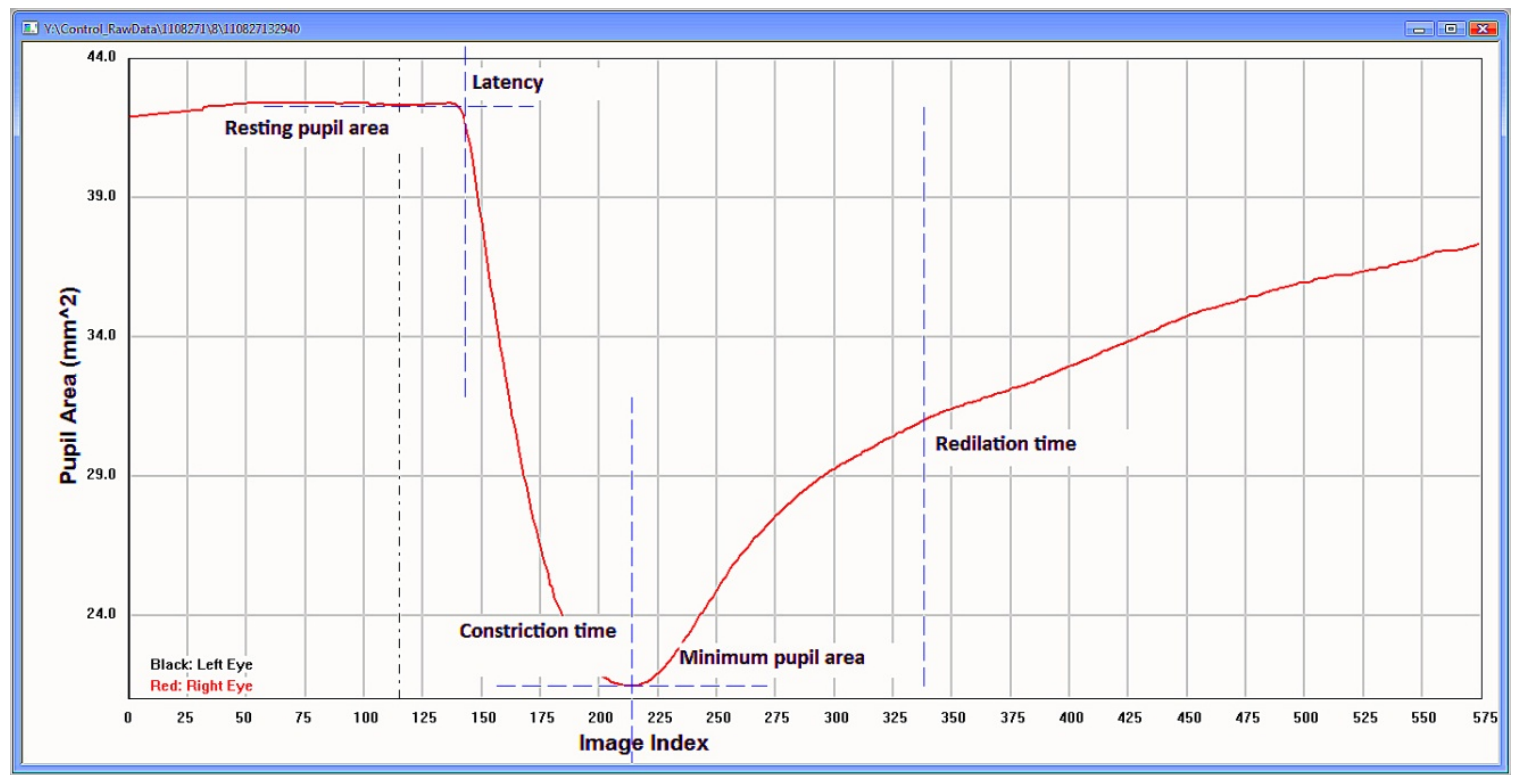

Fig. 2-8: Calipers to mark PLR parameters.

The ability to view the acceleration curve (Fig. 2-9) and the velocity curve (Fig. 2-10) of the pupilogram was implemented. This feature is important, since to measure the latency, identifying the time of maximal acceleration was important.

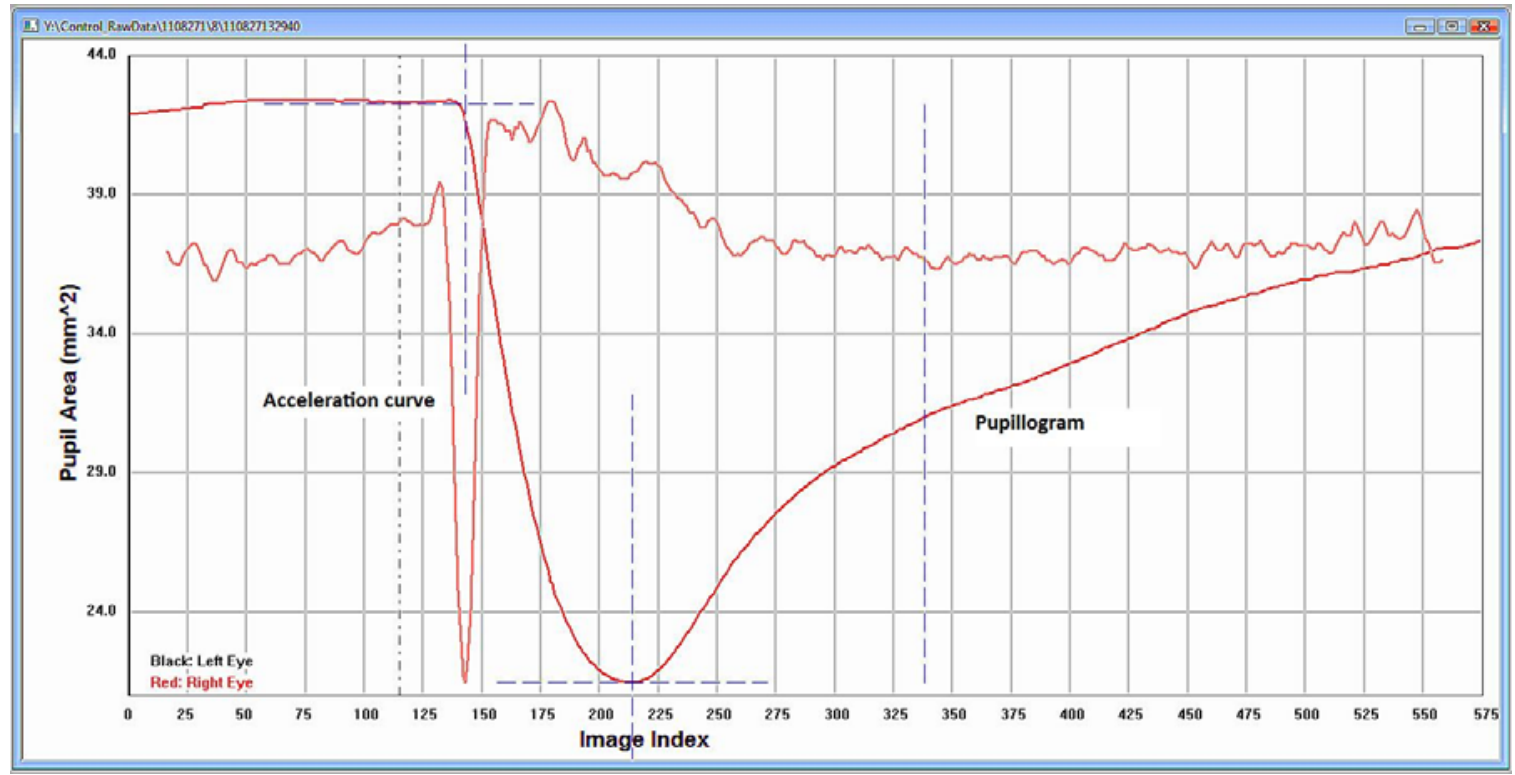

Fig. 2-9: An example acceleration curve of pupilogram 


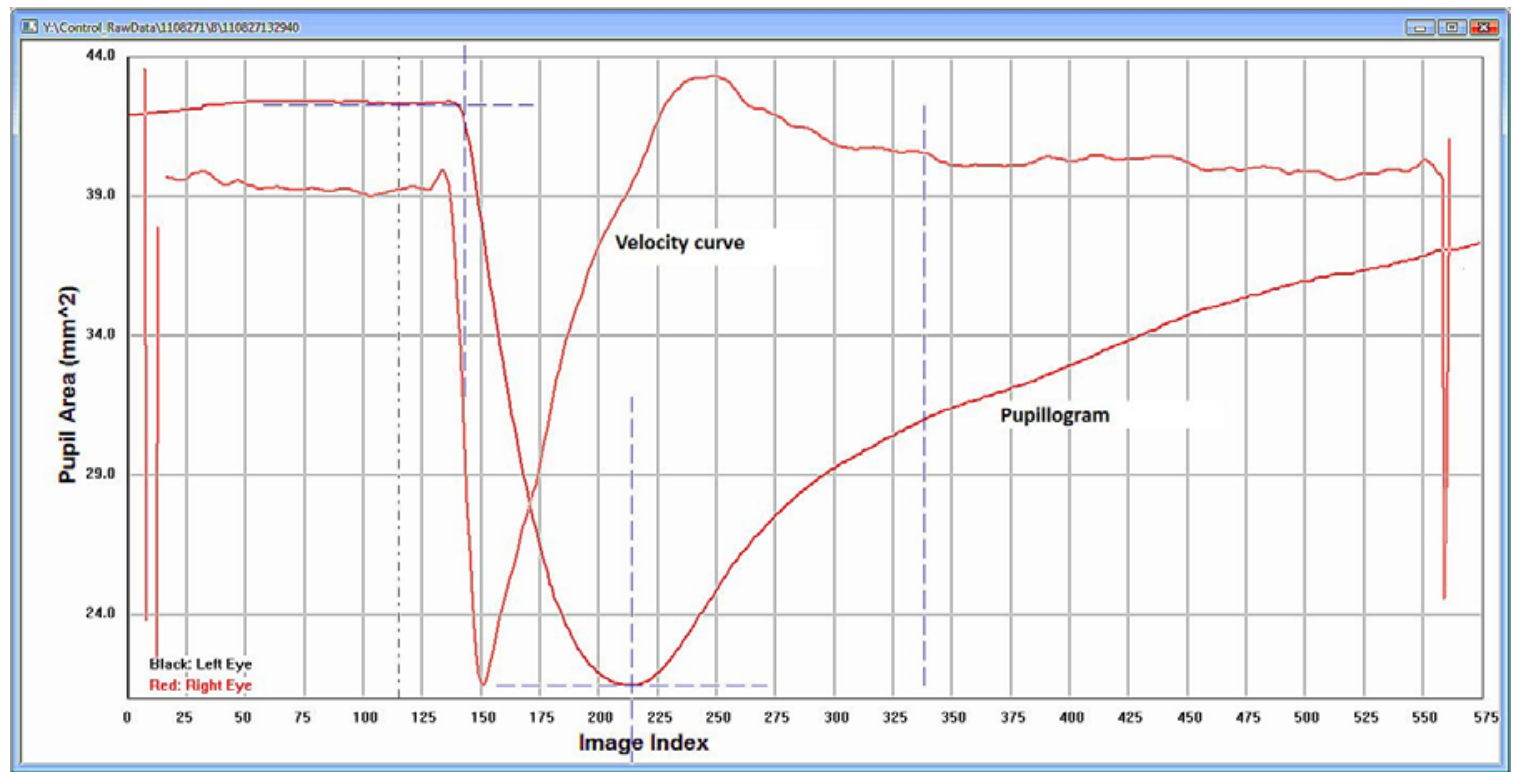

Fig. 2-10: An example velocity curve of pupilogram

The ability to view both normalized pupilogram with respect to the resting pupil diameter and raw pupilogram was implemented too. Raw pupilogram helps observe whether there is anisocoria while the normalized pupilogram is required to observe whether there is constriction anisocoria.

Sometimes segments of pupilogram are noisy due to incorrect ellipse fitting caused by head movement during image acquisition. At such instances, that segment of the pupilogram needs to be reprocessed while keeping the remaining pupilogram intact. Thus the ability to command to reprocess segments of the pupilogram of each eye independently was implemented in the pupil image processing software. These functionalities were helpful when confirming and correcting parameters from noisy pupilograms.

The PLR test for one participant requires multiple measurements because of stimulus changes and repeated measurements at the same stimulus. Therefore, image 
processing consists of processing multiple image sequences, which requires continued repetition of the same task. While performing a large sample study, it is important to automate such repetitive tasks to improve efficiency and reduce time spent on processing. In order to make the repetitive task of image processing faster, multithreading was implemented using Microsoft Foundation Class (MFC) library worker threads. Two controlling functions were defined for the worker thread, one for the left channel images and one for the right channel. For each pupil image in the given image sequence, this function detects pupil boundary and then fits an ellipse to the detected ellipse boundary and finally calculates the area of the fitted ellipse. Thus, when the program has to process images of a single trial, data for each eye is parallel processed. In order to make it automatic, batch process functionality was implemented, which allows the software to automatically process each trial of one subject sequentially as a batch once the subject ID is specified.

\subsection{Conclusions}

The custom-built binocular pupilogram recording system used in this research project can measure PLR in children with high spatial and time resolution. It has the capability of changing stimulus intensity and waveform shape.

The image processing software has proven versatility of processing pupil imaging of different bit resolution, imaging speed, spatial resolution and imaging size; thus, it can be used to process images from other binocular pupilogram recording systems as well. With the implementation of multithreading, batch processing and improvements 
to the GUI, the image processing software was user friendly, efficient and automatic. Therefore, while doing a large sample study, the large work load of image processing could be reduced and delegated. The random Hough transform algorithm implementation can achieve accurate fitting results automatically and with a reasonable computational cost in instances where incomplete pupil images occur and direct least square fitting fails to generate robust results automatically.

To sum up, the developed binocular pupilogram recording system and related image processing software provides a means of measuring pupillary light reflex in children, and does not require special skills to operate. Thus, the system is uniquely qualified for clinical research related to PLR. 


\section{CHAPTER 3}

\section{SIMULTANEOUSLY MEASURED PUPILLARY LIGHT REFLEX AND HEART RATE VARIABILITY IN HEALTHY CHILDREN*}

\subsection{Introduction}

The autonomic nervous system (ANS) is a complex and pervasive system that controls the critical visceral functions of the human body and is involved in many psychophysiological responses. Its two divisions, the sympathetic and parasympathetic systems, act in a complementary manner regulated by the central autonomic network (Levy, 1997). In such a highly integrated organization, ANS dysfunctions are often widespread with symptoms appearing in multiple subsystems. Hence, an interesting question arises: whether an assessment of a specific ANS subsystem may reflect the overall physiological status of the system, and how variations in different measures of ANS correlate with each other (Bär et al., 2009). This study investigated the possible inter-relationship between two specific ANS measures: pupillary light reflex (PLR) and heart rate variability (HRV).

PLR refers to the change of pupil size in response to luminance changes. The pupil size is controlled by two iris muscles, the sphincter and dilator, which produce pupil constriction and dilation, respectively. The sphincter is mainly innervated by the

\footnotetext{
* The material presented in this chapter was published in Physiological Measurement. Daluwatte, C., Miles, J. H. \& Yao, G. 2012. Simultaneously measured pupillary light reflex and heart rate variability in healthy children. Physiological Measurement, 33, 1043-1052.
} 
parasympathetic nervous system (PNS), whereas the dilator is innervated by the sympathetic nervous system (SNS) (Barbur, 2004). The preganglionic neuron synapses in the ciliary ganglion and control the sphincter muscle via the short ciliary nerves (Lowenstein and Loewenfeld, 1950). The preganglionic neuron originates at the Edinger-Westphal (EW) nucleus that receives input from the pretectal olivary nucleus (PON) in the midbrain. The dilator muscles are controlled by long ciliary nerves from superior cervical ganglion that receives input from the cilio-spinal center of Budge (Appenzeller, 1999). The pupil size is determined by the balance between the sympathetic and parasympathetic systems (Fotiou et al., 2000). Abnormal PLR has been observed in many neurological disorders associated with ANS dysfunction (Bremner, 2009) such as panic disorder (Kojima et al., 2004), autism (Fan et al., 2009a) and Parkinson's disease (Giza et al., 2011; Stergiou et al., 2009).

HRV assesses the beat-to-beat variations of the heart rate. Both parasympathetic and sympathetic systems are involved in cardiovascular system regulation. Stimulation of the parasympathetic fibers (vagus nerves) reduces the heart rate, whereas the sympathetic stimulation increases the heart rate through the sinoatrial (SA) node. HRV parameters have been widely applied to evaluate cardiac autonomic functions (Kamath and Fallen, 1993) and have been useful in identifying the ANS function in various allostatic systems (Thayer and Sternberg, 2006). HRV has also been applied in evaluating the ANS dysfunction in disorders such as panic disorder (Yeragani et al., 1993), schizophrenia (Bär et al., 2005, 2007) and sleep disorders (Bonnet and Arand, 1998). 
The possible association between PLR and HRV has recently been investigated by several authors. Correlations between HRV and PLR parameters were found in adults during exercise (Kaltsatou et al., 2011) and in patients with acute schizophrenia (Bär et al., 2008). However, Bär et al. (2009) found limited correlation between specific PLR and HRV parameters in healthy adults $19-64$ years old. It is unclear whether similar relationships may exist in younger subjects as both HRV and PLR are affected by age. Here we report a study that simultaneously measured the PLR and HRV in healthy 8-16 year-old children to further investigate the possible association between measures obtained from pupillary light reflex and heart rate variability (HRV).

\subsection{Methods}

\subsubsection{Participants}

A total of 54 healthy $8-16$ year-old children participated in this study including 27 boys (age $11.0 \pm 2.3$ years) and 27 girls (age $10.9 \pm 2.2$ years) without any known vision, neurological and cardiovascular problems. All were tested at least $1 \mathrm{~h}$ after their last meal. Among the participants, 23 children ( 9 girls and 14 boys) were tested in the morning ( 8 am-12 pm) and the remaining 31 (18 girls and 13 boys) were tested in the afternoon (12 pm-5 pm). All participants and their legal guardians were thoroughly informed of the procedure and consented with a written informed consent as approved by the Institutional Review Board of the University of Missouri. 


\subsubsection{Instrument}

A binocular pupilogram recording system was used to measure the PLR. This system is similar to that reported previously (Fan et al., 2009a, 2009b) except that faster cameras were used to record pupil images at $115 \mathrm{fps}$. The spatial resolution of the system was $35 \mu \mathrm{m} /$ pixel. A $530 \mathrm{~nm}$ green LED provided the light stimulus. The stimulus pulse width was $100 \mathrm{~ms}$. Neutral density filters and LED current were used to control the stimulation intensity.

To obtain HRV, real-time QRS intervals were recorded by using a remote heart rate measuring device (Polar RS800CX, Polar Electro Oy, Finland). A chest strap with an enclosed heart rate sensor and wireless transmitter was wrapped around the participant's chest. The system acquires the ECG at $1 \mathrm{kHz}$ rate. The heart beat QRS signals transmitted from the chest strap were received and recorded by a watch-like device. Multiple studies (Gamelin et al., 2006, 2008; Goodie et al., 2000; Nunan et al., 2009; Porto and Junqueira Jr, 2009) have shown this device to be reliable for the shortterm $\mathrm{R}-\mathrm{R}$ interval measurement and to provide results consistent with a traditional $\mathrm{ECG}$.

\subsubsection{Test Procedure}

Heart rate recording was started 5 min before the PLR test to acquire a baseline reference. The participant remained in a sitting position during the entire test. PLR was first measured in a light-adapted (LA) condition $\left(30 \mathrm{~cd} / \mathrm{m}^{2}\right.$ room illumination). Three optical stimulation intensities were used in LA tests: $69.3,872.1$ and $8721.1 \mathrm{~cd} / \mathrm{m}^{2}$ to induce different amounts of pupillary constriction. Following a 15 min dark adaptation 
$\left(<0.02 \mathrm{~cd} / \mathrm{m}^{2}\right.$ room illumination), dark-adapted (DA) PLR was then measured at a stimulation intensity of $63.1 \mathrm{~cd} / \mathrm{m}^{2}$. At each test condition, the left eye was stimulated first and the right eye was stimulated next. Images of both pupils were recorded for the analysis. The measurements were repeated four times for each condition with a $20 \mathrm{~s}$ interval between measurements. Pupil imaging was started $1 \mathrm{~s}$ before the onset of the 100 ms optical stimulation to obtain baseline pupil size, and was recorded continuously for $4.5 \mathrm{~s}$. Heart rate was continuously recorded during the PLR test and stopped 5 min after completing the PLR test. The entire test procedure is illustrated in Fig. 3-1.

$\begin{array}{rcccccc}\text { Test phase } & 1 & \mathrm{~K} & 2 & 4 & \mathbf{5} & 5 \\ \text { Duration } & 5 \mathrm{~min} & 10 \mathrm{~min} & 15 \mathrm{~min} & 5 \mathrm{~min} & 5 \mathrm{~min} \\ \text { Description } & \text { Pre-test } & \text { LA PLR } & \text { Dark-adaptation } & \text { DA PLR } & \text { Post-test } \\ \text { PLR data } & \text { N/A } & \text { YES } & \text { N/A } & \text { YES } & \text { N/A } \\ \text { HRV data } & \text { YES } & \text { YES } & \text { YES } & \text { YES } & \text { YES }\end{array}$

Fig. 3-1: An illustration of the test procedure used in this study.

\subsubsection{Data Analysis}

The pupilogram (Fig. 1-2) was constructed by extracting the pupil size from acquired pupil images as described in detail previously (Fan et al., 2009a, 2009b). As in our previous studies, the PLR parameters listed in Table 3-1 were measured in this study to characterize PLR responses. PLR parameters obtained from both eyes during the four repeat measurements were averaged to calculate the mean value at any given condition. 
Table 3-1: PLR parameters measured in this study.

\begin{tabular}{|c|c|}
\hline Symbol (unit) & Definition \\
\hline$\overline{D_{0}(\mathrm{~mm})}$ & "Base pupil diameter": pupil diameter before stimulus onset \\
\hline$D_{\min }(m m)$ & "Minimal pupil diameter": pupil diameter before stimulus onset \\
\hline$\Delta \mathrm{A}_{\%}$ & "Relative constriction amplitude": $\left(D_{0}^{2}-D_{\min }^{2}\right) / D_{0}^{2}$ \\
\hline$t_{L}(m s)$ & $\begin{array}{l}\text { "Constriction latency": the elapsed time between light stimulus and } \\
\text { beginning of constriction }\end{array}$ \\
\hline $\mathrm{t}_{\mathrm{c}}(\mathrm{ms})$ & $\begin{array}{l}\text { "Constriction time": the time interval from beginning of constriction to } \\
\text { the maximal constriction }\end{array}$ \\
\hline$t_{R}(m s)$ & $\begin{array}{l}\text { "Redilation time": the time interval between maximal constriction and } \\
\text { recovery to half of the constriction }\left(D_{0}^{2}-D_{\min }^{2}\right) / 2\end{array}$ \\
\hline $\mathrm{v}_{\mathrm{c}}(\mathrm{mm} / \mathrm{s})$ & $\begin{array}{l}\text { "Constriction velocity": average velocity of the relative constriction } \\
\left(D_{0}-D_{\min }\right) / t_{c}\end{array}$ \\
\hline $\mathrm{V}_{\mathrm{R}}(\mathrm{mm} / \mathrm{s})$ & $\begin{array}{l}\text { "Recovery velocity": average velocity of the relative recovery } \\
\left(D_{0}-D_{\min }\right) /\left(2 t_{R}\right)\end{array}$ \\
\hline
\end{tabular}

HRV was assessed using both time domain and frequency domain methods (Malik et al., 1996). As shown in Table 3-2, two time domain parameters were calculated: SDNN and rMSSD. To be consistent with the most recent studies on HRV, two frequency domain parameters were analyzed: the normalized $\mathrm{HF}$ and the LF/HF ratio. The frequency domain power spectrum was analyzed by using fast Fourier transform (FFT) as described by Malik et al. (1996). To study the potential effect of the PLR tests on HRV parameters, HRV was analyzed in five testing phases: before PLR test (5 min), LA test (10 min), dark adaptation (15 min), DA test (5 min) and after PLR test (5 $\min )$. 
Table 3-2: HRV parameters measured in this study.

\begin{tabular}{ll}
\hline Symbol (unit) & \multicolumn{1}{c}{ Definition } \\
\hline AHR (bpm) & Average heart rate \\
SDNN (ms) & Standard deviation of normal RR intervals \\
rMSSD (ms) & Root mean square successive difference of the RR intervals \\
HF $_{N}$ & Normalized Powers of HF band (HF, 0.15 - 0.4 Hz), i.e., the relative \\
& powers of HF band when removing Very Low frequency (VLF, 0 - 0.04 \\
& Hz) band power from total power. HF power/( Total power -VLF power \\
& The ratio of LF/HF power, $(\mathrm{LF}, 0.04-0.15 \mathrm{~Hz})$ \\
\end{tabular}

The distributions of PLR and HRV parameters were verified to conform to normal distributions using the Kolmogorov-Smirnov test. For each PLR and HRV parameter, the analysis of covariance (ANCOVA) using the PROC MIXED procedure in SAS (Version 9.2, SAS Institute Inc., Cary, NC, USA)was applied to examine the effects of test conditions, gender and age. $p$-values were Bonferroni-corrected appropriately using the number of stimulus conditions for PLR and the number of testing phases for HRV. Paired t-test was used to confirm PLR test phase effects on HRV parameter and corresponding effect size (d statistic) was calculated using the method described by Nakagawa and Cuthill (2007) and values of $0.2,0.5$ and 0.8 were considered small, medium and large, respectively (Cohen, 1992). To study the association between PLR and HRV parameters, linear correlations between them were first analyzed with Pearson product moment correlation (PROC CORR procedure in SAS). To investigate whether variations in PLR parameters can be explained by a combination of HRV parameters or vice versa, multilinear regression (PROC REG procedure in SAS) was applied with HRV (or PLR) parameters treated as dependent variables and PLR (or HRV) parameters treated as independent variables. Moreover, the explanatory factor analysis (PROC FACTOR 
procedure in SAS) was performed on the overall data set to study the potential relationships between PLR and HRV parameters using oblique matrix rotation. The factor analysis reduces a multivariate system to a system with a fewer number of dimensions by categorizing highly correlated dependent variables into a single factor.

\subsection{Results}

\subsubsection{Pupillary Light Reflex}

Table 3-3: Summary of PLR results.

\begin{tabular}{lllll}
\hline & \multicolumn{4}{c}{ Stimulation intensity $\left(\mathbf{c d} / \mathrm{m}^{2}\right)$} \\
& LA 69.3 & LA 872.1 & LA 8721.1 & DA 63.1 \\
\cline { 2 - 5 } $\begin{array}{llll}\text { Resting pupil diameter (mm) } \\
\text { Relative constriction }\end{array}$ & $12.8 \pm 6.0$ & $27.0 \pm 7.4$ & $40.8 \pm 7.89$ & $4.5 \pm 0.8$ \\
amplitude (\%) & & & & \\
Latency (ms) & $269.3 \pm 26.6$ & $236.1 \pm 15.8$ & $211.8 \pm 13.5$ & $239.3 \pm 15.9$ \\
Constriction Time (ms) & $368.6 \pm 71.8$ & $398.5 \pm 56.6$ & $463.4 \pm 52.8$ & $575.6 \pm 52.3$ \\
Redilation Time (ms) & $417.6 \pm 74.7$ & $501.7 \pm 109.9$ & $610.7 \pm 135.8$ & $817.8 \pm 172.0$ \\
Constriction velocity (mm/s) & $1.0 \pm 0.5$ & $2.0 \pm 0.7$ & $2.8 \pm 0.8$ & $2.8 \pm 0.8$ \\
Redilation velocity (mm/s) & $0.5 \pm 0.2$ & $0.8 \pm 0.3$ & $1.1 \pm 0.3$ & $1.0 \pm 0.0$ \\
\hline
\end{tabular}

The results are represented as group mean \pm standard deviation.

Table 3-3 shows the mean values and standard deviations of all measured PLR parameters. The initial pupil diameter $D_{0}$ increased from $6.6 \pm 0.6 \mathrm{~mm}$ in LA to $7.5 \pm 0.8$ $\mathrm{mm}$ in $\mathrm{DA}$. The relative constriction amplitude $\Delta \mathrm{A}_{\%}$ increased from $12.8 \% \pm 6.0 \%$ at $\mathrm{LA}$ $69.3 \mathrm{~cd} / \mathrm{m}^{2}$ to $40.6 \% \pm 7.3 \%$ at LA $8721.1 \mathrm{~cd} / \mathrm{m}^{2}$. The PLR latency was between 200 to 300 ms. In LA tests, PLR latency decreased with stimulus intensity ( $F$ value $=236.0, p<0.0001$ in ANOVA test for a linear trend). $\triangle \mathrm{A}_{\%}$ was similar at $L A 8721.1 \mathrm{~cd} / \mathrm{m}^{2}$ and $D A 63.1$ $\mathrm{cd} / \mathrm{m}^{2}$, but the DA latency was $\sim 30 \mathrm{~ms}$ longer. Average constriction velocity $\mathrm{v}_{\mathrm{c}}$ ranged 
from 1.0 to $2.8 \mathrm{~mm} / \mathrm{s}$ at the four stimulation conditions; whereas the average redilation velocity $\mathrm{v}_{\mathrm{R}}$ was $0.5-1.0 \mathrm{~mm} / \mathrm{s}$.

\subsubsection{Heart Rate Variability}

The HRV parameters are shown in Table 3-4. The average heart rate was $\sim 90$ bpm in our study population. The time domain HRV parameter SDNN was $\sim 65-72 \mathrm{~ms}$ on average and the rMSSD ranged between 33 and 39 ms. The frequency domain HRV parameters were HF normalized power ( 22-32) and LF/HF ratio ( 2-4).

Table 3-4: Summary of HRV results.

\begin{tabular}{llllll}
\hline & & \multicolumn{3}{c}{ Testing phase } \\
& $\begin{array}{l}\text { Before PLR } \\
\text { test }\end{array}$ & $\begin{array}{l}\text { During LA } \\
\text { PLR test }\end{array}$ & $\begin{array}{l}\text { During DA } \\
\text { period }\end{array}$ & $\begin{array}{l}\text { During DA } \\
\text { PLR test }\end{array}$ & $\begin{array}{l}\text { After PLR } \\
\text { test }\end{array}$ \\
\cline { 2 - 6 } Heart rate & $89.9 \pm 12.1$ & $90.1 \pm 12.1$ & $92.7 \pm 12.1$ & $91.0 \pm 13.2$ & $93.5 \pm 13.2$ \\
(bpm) & $67.6 \pm 27.0$ & $70.2 \pm 23.9$ & $67.9 \pm 29.1$ & $71.5 \pm 28.5$ & $65.2 \pm 25.0$ \\
SDNN (ms) & $38.9 \pm 20.3$ & $36.7 \pm 16.9$ & $34.1 \pm 17.7$ & $36.4 \pm 18.6$ & $33.2 \pm 16.9$ \\
rMSSD (ms) & $31.8 \pm 11.0$ & $23.1 \pm 7.1$ & $26.9 \pm 10.1$ & $22.3 \pm 8.2$ & $24.9 \pm 10.1$ \\
HF $_{\text {(\%) }}$ & $2.6 \pm 1.4$ & $3.8 \pm 1.6$ & $3.2 \pm 1.4$ & $4.1 \pm 2.1$ & $3.7 \pm 2.3$ \\
LF/HF ratio & 2.4 & &
\end{tabular}

The results are represented as group mean \pm standard deviation.

\subsubsection{Interrelationship between PLR and HRV}

As shown in Table 3-4, the average heart rate, SDNN and rMSSD measured during different PLR testing phases were not significantly different (ANCOVA $F_{4,262}=1.06$, 0.52 and $0.86, p=0.38,0.72$ and 0.48 , respectively). In fact, the HRV parameters obtained during different testing phases were highly correlated with each other. The heart rate had the highest correlation coefficient among all parameters. Table 3-5 shows 
that the correlation weakened as the measurement phase became distinct in time, i.e. the $r$ value decreased from 0.95 between phase 1 and 2 ( 5 min interval) to 0.88 between phase 1 and 5 (25 min interval).

Table 3-5: Correlations between average heart rate measured in different testing phases.

\begin{tabular}{lccccc}
\hline & Before Test & LA Test & DA & DA Test & After Test \\
\cline { 2 - 5 } Before Test & $1.00^{*}$ & $0.95^{*}$ & $0.91^{*}$ & $0.92^{*}$ & $0.88^{*}$ \\
LA Test & & $1.00^{*}$ & $0.94^{*}$ & $0.95^{*}$ & $0.90^{*}$ \\
DA & & $1.00^{*}$ & $0.93^{*}$ & $0.91^{*}$ \\
DA Test & & & & $1.00^{*}$ & $0.90^{*}$ \\
After Test & & & & & $1.00^{*}$ \\
\hline
\end{tabular}

The results are represented as Pearson's r-value. " $p<0.0001$

However, the correlation of frequency domain HRV among different testing phases was not as high as the time domain parameters. The LF/HF ratio had the least correlation r-values (0.54-0.87) among different testing phases. In fact, the ANCOVA model indicated that the PLR testing phase had a significant fixed effect on the LF/HF ratio and HF normalized power (ANCOVA $\mathrm{F}_{4,262}=6.74$ and 8.52, $p<0.0001$ and $p<$ 0.0001). As shown in Fig. 3-2 (Table 3-6), HF normalized power was significantly lower during the two PLR testing phases (both LA and DA tests) than during the three resting periods (before the PLR test, dark adaptation and after the PLR test), while the LF/HF ratio was significantly higher.

The Pearson product moment correlation did not show any significant correlation between simultaneously measured PLR parameters and HRV parameters $(p>$ $0.05, r<0.5$ for all the correlations). Further multilinear regression analysis concluded 
that no significant model could be developed to explain variations in PLR parameters by using HRV parameters, nor vice versa.
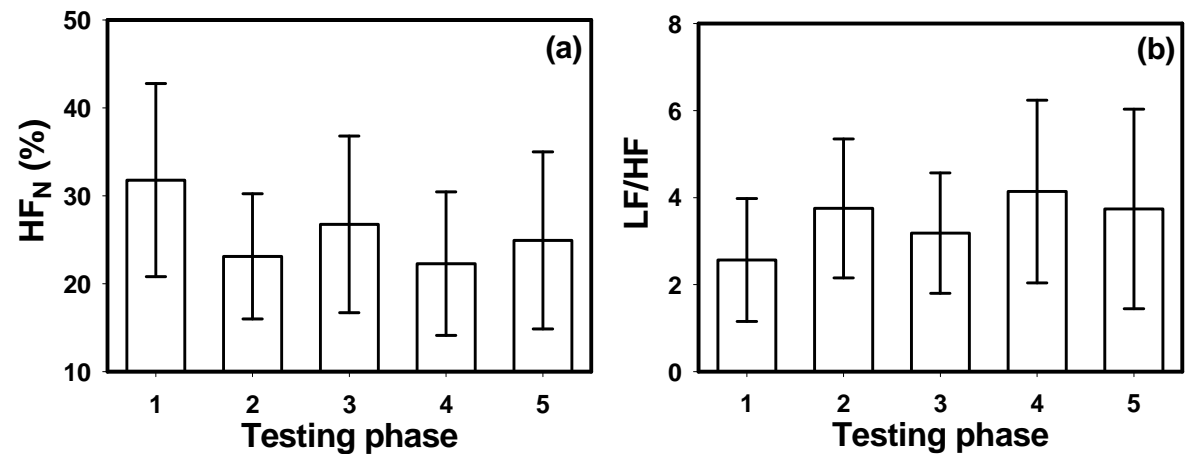

Fig. 3-2: A comparison of frequency domain HRV parameters during different testing phases.

(a) normalized HF power, (b) LF/HF ratio. $p$-values shown were obtained from paired ttest. The testing phases are numbered as 1: before PLR test, 2: during LA PLR, 3: during dark adaptation, 4: during DA PLR, 5: after PLR test. The error bars indicate the standard deviation. Paired t-test statistics shown on Table 3-6.

Table 3-6: Paired t-test results and corresponding effect sizes for comparison of frequency domain HRV parameters during different testing phases.

\begin{tabular}{ccrrr}
\hline & & \multicolumn{1}{c}{$\boldsymbol{t}_{\mathbf{5 3}}$} & \multicolumn{1}{c}{$\boldsymbol{p}$ value } & Effect size (d) \\
\cline { 3 - 5 } $\mathbf{H F}_{\mathbf{N}}$ & $1 \rightarrow 2$ & 8.89 & $<0.0001$ & 0.8 \\
& $2 \rightarrow 3$ & -3.19 & 0.024 & 0.4 \\
& $3 \rightarrow 4$ & 3.59 & 0.0007 & 0.5 \\
& $4 \rightarrow 5$ & -2.13 & 0.0378 & 0.3 \\
LF/HF & $1 \rightarrow 2$ & -11.08 & $<0.0001$ & 0.8 \\
& $2 \rightarrow 3$ & 3.24 & 0.0021 & 0.4 \\
& $3 \rightarrow 4$ & -4.21 & 0.0001 & 0.5 \\
& $4 \rightarrow 5$ & 1.51 & 0.1374 & 0.2 \\
\hline
\end{tabular}

Using the exploratory factor analysis with oblique matrix rotation (OBVARIMAX in SAS), the multivariate PLR and HRV dataset was reduced to a system of four factors which were uncorrelated with each other (inter-factor correlations $r<0.3$ ). These four factors were determined such that the total variance of the dataset (proportion $=1$ ) can be explained by the resulting system with a reduced dimension and also using the scree 
plot to identify the last substantial drop which is after factor 4 (Fabrigar et al., 1999). The factor loading after oblique matrix rotation (OBVARIMAX in SAS) is shown in Table 3-7, where each factor loading represents the correlation between a PLR or HRV parameter and the identified factor. Factors 1 and 2 were mainly attributed to loadings from HRV parameters. The heart rate and time domain HRV parameters (SDNN and rMSSD) had the highest correlation with factor 1 , whereas the two frequency domain $\mathrm{HRVs}$, LF/HF and $\mathrm{HF}_{\mathrm{N}}$, had the highest correlation with factor 2. PLR parameters were the major contributors to factors 3 and 4 . Factor 3 had a high loading from $t_{C}$ and $t_{R}$, while factor 4 had moderately high correlations with $t_{L}$ and $D_{0}$. The reliability of each variable was evaluated by analyzing "Cronbach's coefficient of alpha when deleted" with 'PROC CORR'. The results indicated that the heart rate should be removed from factor 1. In addition, a "Cronbach's coefficient of alpha" of 0.7 was used as the acceptable threshold to evaluate the reliability of the entire factor system. The results indicated that the final solution should only consist of factors 1 and 3 , where factor 1 represented the HRV system and factor 3 represented the PLR system. Communalities of selected parameters over the final factor solution were above 0.8 for all the parameters selected in the factor solution. Such consistently high communalities suggested that the sample size of the design had a minimal effect on the factor solution (MacCallum et al., 1999). The numbers shown in Table 3-7 were calculated by using PLR data obtained at LA $8721.1 \mathrm{~cd} / \mathrm{m}^{2}$ and HRV data obtained during the LA testing phase. However, the same conclusion was reached for all other testing conditions. 
Table 3-7: Factor loadings for the entire data set by using factor analysis.

The numbers represent correlation between PLR/HRV parameters and the identified factors.

\begin{tabular}{|c|c|c|c|c|c|}
\hline & & Factor 1 & Factor 2 & Factor 3 & Factor 4 \\
\hline \multirow{5}{*}{ 卒 } & RMSSD & 0.93 & 0.19 & 0.03 & -0.02 \\
\hline & SDNN & 0.95 & -0.07 & 0.00 & -0.02 \\
\hline & AHR & -0.81 & -0.02 & -0.06 & 0.02 \\
\hline & LF/HF ratio & -0.02 & -0.90 & 0.10 & 0.01 \\
\hline & $\mathrm{HF}_{\mathrm{N}}$ & 0.01 & 0.94 & 0.03 & 0.01 \\
\hline \multirow{4}{*}{$\frac{\mathscr{a}}{a}$} & Constriciton Time & -0.01 & 0.00 & 0.93 & -0.07 \\
\hline & Redialtion Time & 0.02 & -0.05 & 0.91 & 0.09 \\
\hline & Latency & 0.02 & -0.03 & 0.04 & 0.42 \\
\hline & Initial Pupil Diameter & -0.02 & 0.02 & -0.02 & 0.34 \\
\hline
\end{tabular}

\subsection{Discussion}

The PLR parameters obtained in this study are similar to those reported previously (Fan et al., 2009a) in a similar age group at similar stimulus conditions. Similarly, the time domain HRV parameters obtained in this study are consistent with those reported in previous studies (Umetani et al., 1998). Our mean $\mathrm{HF}_{\mathrm{N}}(\%)$ is smaller than that reported by Gamelin et al. (2008) in boys younger than 11 years in a supine position. We think that these differences can be attributed to the seated position used in our study. It is known that HRV measured at a supine position has a higher $\mathrm{HF}_{\mathrm{N}}(\%)$ and smaller LF/HF than that measured at a standing position (Montano et al., 1994). The HRV parameters obtained in seated position are between those obtained at supine and standing positions (Chan et al., 2007).

Because $43 \%$ of participants were tested in the morning and the rest were tested in the afternoon, we examined the potential effect of the different time of day of the test. The results showed no significant difference between the PLR and HRV 
measurements obtained in children tested in the morning and those tested in the afternoon (t-test, $p>0.15$ ). The HRV results (Table 3-4) measured in phases 2 and 3 (Fig. 3-1) had longer measurement times (10 $\mathrm{min}$ and $15 \mathrm{~min}$, respectively) than the other three phases (5 min each). To investigate whether such a difference may lead to different HRV values, we divided the 10 min LA test period into two 5 min segments and calculated HRV parameters in each segment. The obtained HRV parameters in the two 5 min segments were similar to each other (paired t-test, $p>0.28$ ) and were also similar to the one calculated over the entire 10 min period. The same results were obtained for the HRV parameters obtained in the 15 min DA period.

The frequency domain HRV parameters were clearly affected by the PLR testing phases. The mean results (Table $3-4$ ) indicate that $\mathrm{HF}_{\mathrm{N}}$ decreased $27.3 \%$ and $\mathrm{LF} / \mathrm{HF}$ increased $45.9 \%$ when going from 'before test' to PLR test. This trend is similar to posture-induced HRV changes that are related to sympathetic activity caused by orthostatic stress (Mukai and Hayano, 1995; Montano et al., 1994; Yeragani et al., 1993). Because the participant was slightly inclined forward $\left(\sim 15^{\circ}\right)$ during the PLR tests, it is possible that such a posture change led to elevated sympathetic activity due to increased muscle stress. In addition, the PLR test itself may induce some task-related stress. Delaney and Brodie (2000) reported that psychological stress can decrease highfrequency HRV and increase low-frequency HRV. Vagal tone and respiratory sinus arrhythmia (RSA) are the principal contributors to the high-frequency power of HRV, while both vagal and sympathetic tones influence the low-frequency component (Berntson et al., 1997). Overall the observed changes in frequency domain HRV 
parameters suggest lower vagal tone and increased sympathetic modulation during the PLR test.

PLR parameters and HRV parameters are categorized as independent and uncorrelated factors according to the factor analysis. The SDNN and RMSSD were closely aligned only with factor 1 and had very low loading on other factors (Table 3-7). Similarly, the constriction time and redilation time were aligned only with factor 3 . $\mathrm{LF} / \mathrm{HF}$ and $\mathrm{HF}_{\mathrm{N}}$ were highly aligned with factor 2 with high loading. However, with a smaller loading, PLR latency and initial pupil diameter were best aligned with factor 4 . Therefore, the data clearly indicated a lack of association between PLR and HRV. This observation may be attributed to the fact that healthy, typically developing children were tested in this study. In this group, many PLR parameters were quite consistent with very small between-subject variation. Notably, the coefficient of variation of PLR latency was $<10 \%$, in agreement with the fact that latency did not contribute to the four-factor system derived from the common factor analysis.

The lack of association between PLR and HRV can be further corroborated by the different gender effect in PLR and HRV parameters. In agreement with a previous report by Krishnan et al (2009) in a large group of children of similar age range, our data showed that girls had a significantly higher heart rate than boys. A higher heart rate indicates stronger sympathetic influence (Malik et al., 1996). We observed a trend of smaller resting pupil size in girls although the difference did not reach statistical significance. The same difference was reported by (Fan et al., 2009b) as statistically significant in individuals with a tightly controlled age range. A smaller resting pupil size 
may suggest a stronger parasympathetic modulation or a weaker sympathetic modulation (Barbur, 2004). Summarizing the above comparisons, it is clear that gender effects in PLR and HRV do not completely corroborate with each other.

Although PLR and HRV are part of an integrated ANS, individual neurological pathways behave differently. The natural variations in an individual system may not affect other subsystems. However, this conclusion may not hold true for some disorders associated with ANS dysfunctions where pervasive ANS changes exist in multiple subsystems. For example, significant correlations between PLR and HRV were found in patients with acute schizophrenia (Bär et al., 2008).

\subsection{Conclusion}

We measured PLR and HRV simultaneously in age- and gender-matched healthy children 8-16 years old. The gender effect was observed in both HRV and PLR parameters. However, the significant age effect was observed in HRV parameters but not in PLR. We found that the frequency domain HRV parameters were significantly different in different PLR testing phases, likely due to the psychological or/and physiological stress induced by the PLR tests. Using both the regression analysis and factor analysis, we conclude that variations of PLR and HRV are not associated in healthy children. However, this conclusion might be altered in the case of ANS disorders or other situations when pervasive changes might appear in multiple different ANS subsystems. Nevertheless, PLR and HRV may provide a complementary assessment of different aspects of the overall autonomic nervous system. 


\section{CHAPTER 4}

\section{AGE-DEPENDENT PUPILLARY LIGHT REFLEX PARAMETERS IN}

\section{CHILDREN ${ }^{*}$}

\subsection{Introduction}

The American Academy of Pediatrics (AAP) estimates that $12 \%$ to $16 \%$ of children have some form of developmental disorders (Sand et al., 2005). Substantial clinical evidence supports that early intervention leads to improved functioning. Early detection is essential to ensure early intervention (Sand et al., 2005). In the United States developmental screening is presumed to be done in the pediatrician's or family doctor's office using one or more screening questionnaires (Duby et al., 2006). Unfortunately, this practice is neither consistent nor universal, which leads to a considerable lag in the diagnosis for children with developmental disabilities (Sand et al., 2005). In addition, behavioral symptoms usually lag behind the underlying neurophysiological changes. Therefore, there is a need for an objective measure that can accurately track normal neurodevelopment progress in children.

Pupillary light reflex (PLR) is tested by measuring pupil size change in response to a short light flash. The size of the pupil is controlled by two antagonistic iris muscles: the

\footnotetext{
* Part of the material presented in this chapter was published in IEEE Xplore: Daluwatte, C., Miles, J. H., Christ, S. E., Beversdorf, D. Q., Lofgreen, A., Berliner, N. \& Yao, G. Year. Age-dependent pupillary light reflex parameters in children. In: Engineering in Medicine and Biology Society (EMBC), 2012 Annual International Conference of the IEEE, 2012 San Diego, CA. 3776-3779.
} 
sphincter and the dilator that are innervated by different neurological systems (Fan and Yao, 2011). Photoreceptors in the retina detect and convey the sensory information about retinal illumination to the pretectal olivary nucleus (PON) via optic nerves. The PON projects at the Edinger-Westphal (EW) nucleus (Simpson et al., 1988) which then projects to the ciliary ganglion to control the sphincter muscle via the short ciliary nerves (Neuhuber and Schrödl, 2011; Barbur, 2004). The neurological pathway related to pupil dilation is still not well understood (Neuhuber and Schrödl, 2011). The dilator muscle receives control from the superior cervical ganglion via the long ciliary nerves. The cilio-spinal center of Budge is found to project to the superior cervical ganglion (Appenzeller, 1999).

PLR responses can be altered by dysfunctions in the PLR pathway. In fact, abnormal PLRs have been previously reported in several types of neurological disorders. Fan el al. (2009a) reported prolonged PLR latency, smaller relative constriction and lower constriction velocity related to autism spectrum disorders (ASD). Giza el al. (2011) reported prolonged latency, reduced amplitude, maximum constriction velocity and maximum acceleration associated with Parkinson's disease. Fotiou et al. (2007a) reported atypical PLR associated with Alzheimer's disease, where all parameters except baseline and minimum pupil diameters were affected.

To develop an effective screen for neurodevelopment disorders, it is important to first understand neurodevelopment in typically developing children. Several studies have been conducted to examine the normal neurodevelopmental progress of the visual system in children by using visual evoked potentials (VEP) (Carrillo-De-La-Peña et al., 
1999; Lenassi et al., 2008). Age effects in PLR have been previously studied by few groups. Bitsios et al. (1996) measured PLR in young (mean age 19.5 years) and old groups (mean age 69 years) and found that while there was no difference in latency, amplitude, maximum constriction/redilation velocity and redilation time were affected by age. Similar to findings by Bitsios et al. (1996), Fotiou et al. (2007b) too found that PLR latency was not affected by age in a large population of young (18-50 years) and old (51-81 years) people. Fotiou et al. (2007b) also reported that baseline pupil size, maximum constriction velocity and acceleration changed with age. Daneault et al. (2012) studied the steady state pupil constriction to light in two groups: young (mean age $22.8 \pm 4$ years) and old (mean age $61 \pm 4.4$ years) and reported no significant age effect on the steady state pupil constriction when expressed with respect to baseline pupil size. Thus, Daneault et al. (2012) concluded that the age effects reported by Bitsios et al. (1996) have a large contribution from other factors rather than light sensitivity. A recent report demonstrated the potential of using PLR to examine the visual system development in preterm babies (Cocker et al., 2005). However, no comprehensive study has been conducted to investigate age related profiles of PLR parameters in children.

Here we report our results of PLR tests in over 100 typically developing children from 6 to 17 years old. Our results revealed a significant age effect in PLR parameters, particularly the PLR latency and resting pupil diameter. A similar trend was not observed in a group of age-match children with neurodevelopmental disorders. 


\subsection{Methods}

\subsubsection{Test Procedure}

PLR data were obtained in 107 healthy children 6 to 17 years old (mean age $10.9 \pm 2.9$ years) without any known visual or neurological problems. There were 79

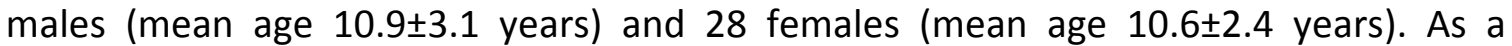
comparison, PLR data were also examined in 176 children (mean age 10.5 \pm 3.1 years, 150 males and 26 females) with several different types of neurodevelopmental disorders including autism spectrum disorders (147), Down syndrome (7), Fragile X syndrome (5), Prader-Willi syndrome (1), and the remainder with idiopathic intellectual impairment. This group of participants was recruited through the Thompson Center for Autism and Neurodevelopmental Disorders at the University of Missouri. Written consents were obtained from all participants and their legal guardians as approved by the Institutional Review Board of University of Missouri.

PLR was measured in both light adapted (LA) (room luminance of $30 \mathrm{~cd} / \mathrm{m}^{2}$ ) and dark adapted (DA) $\left(<0.02 \mathrm{~cd} / \mathrm{m}^{2}\right.$ room luminance) conditions. The intensities used as optical stimulation for PLR were $63.1 \mathrm{~cd} / \mathrm{m}^{2}$ in dark-adaptation and $69.3 \mathrm{~cd} / \mathrm{m}^{2}, 872.1$ $\mathrm{cd} / \mathrm{m}^{2}, 8721.1 \mathrm{~cd} / \mathrm{m}^{2}$ in light adaptation. For each stimulus condition, PLR responses from both eyes were measured when one eye was stimulated. The measurements were repeated four times for each condition with an approximately 30s interval between two consecutive measurements. Imaging was started 1s before the stimulation to gather the resting pupil size. After the LA test, all participants stayed in the dark room for 15 minutes for the pupils to naturally dilate before starting the DA test. 


\subsubsection{Data analysis}

The Analysis of Covaraince (ANCOVA) was applied in SAS to examine the effects of age and test conditions on each PLR parameter. Follow up analysis of variance (ANOVA) was performed to verify the age effect for a linear relationship. PLR parameters were verified for normal distribution using the Kolmogorov-Smirnov test. $p<0.05$ was considered as significant.

\subsection{Results}

As expected, in typically developing children the resting pupil diameter was larger in dark adaptation $(7.44 \pm 0.77 \mathrm{~mm})$ than in light adaptation $(6.58 \pm 0.61 \mathrm{~mm})$ as shown in Fig. 4-1. The resting pupil diameter increased with age significantly before 12 years old $\left(F_{6,135}=2.67, p=0.018\right)$. From 6 to 12 years old, the mean resting pupil diameter increased $8.0 \%$ in LA and $13.2 \%$ in DA (Fig. 4-1). The ANOVA test for a linear trend further confirmed that the age effect was significant ( $p=0.047$ at LA and $p=0.003$ at DA). At the same stimulus intensity, the PLR constriction amplitude was larger in darkadaption whereas the constriction/redilation times were longer and latency was shorter. In light-adapted tests, as stimulus intensity increased from $69.3 \mathrm{~cd} / \mathrm{m}^{2}$ to $8721.1 \mathrm{~cd} / \mathrm{m}^{2}$, PLR latencies decreased 21.85\%; constriction and redilation times increased $25.30 \%$ and $48.15 \%$, respectively; and relative constriction amplitude increased from $11.76 \pm 5.54 \%$ to $40.75 \pm 7.23 \%$.

The ANCOVA model suggested a significant age effect on several PLR parameters. In children from 6 to 8 years old, the age effect was significant for 
constriction amplitude $\left(F_{3,132}=3.48, p=0.018\right)$. PLR constriction increased with age in children younger than 8 years old and reached a plateau thereafter (Fig. 4-2a) at all stimulation conditions except the one at LA $69.3 \mathrm{~cd} / \mathrm{m}^{2}$. However, the linear increasing trend at a young age $(<8$ years) was significant only with the maximal stimulus at LA 8721.1 $\mathrm{cd} / \mathrm{m}^{2}\left(\mathrm{~F}_{1,21}=5.70, p=0.027\right)$. The PLR constriction time and the redilation time did not show an effect with age.

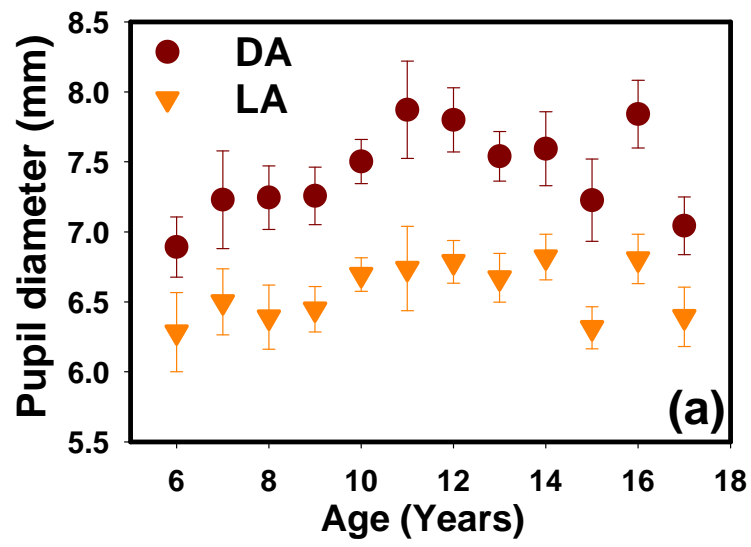

Fig. 4-1: The age effect in resting pupil diameter in children with typical development. LA - in the light adapted and DA - in the dark adapted environment. The error bars indicate the standard error.
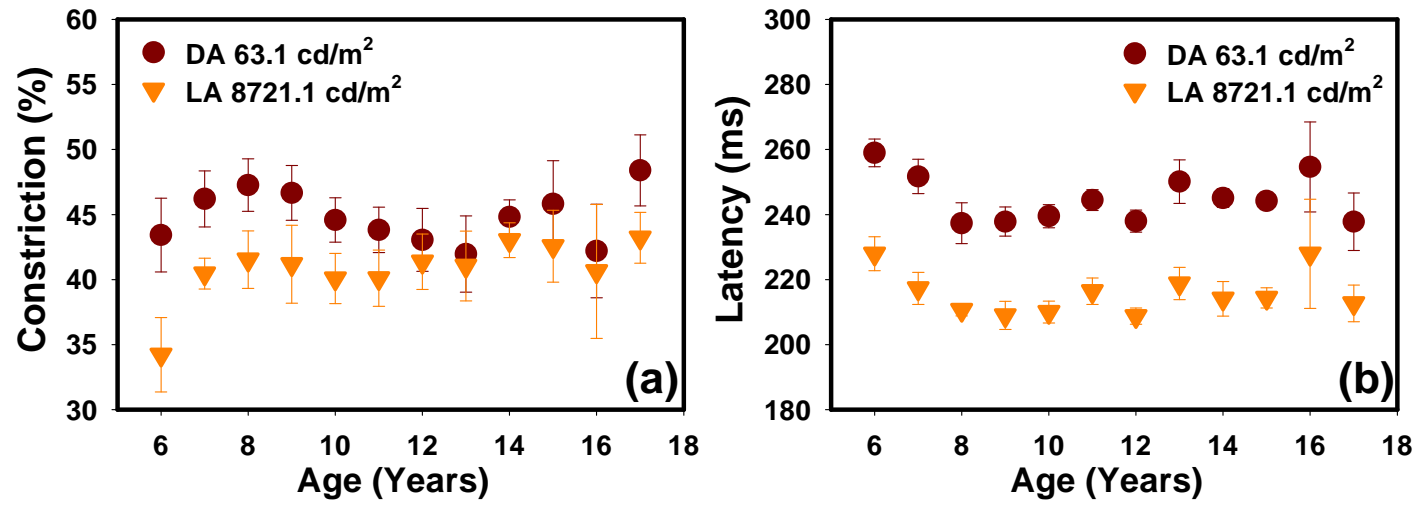

Fig. 4-2: The age effect in PLR parameters obtained in children with typical development from 6 to 17 years old.

(a) Relative constriction amplitude, (b) latency measured in the light adapted (LA) $8721.1 \mathrm{~cd} / \mathrm{m}^{2}$ and dark adapted (DA) $63.1 \mathrm{~cd} / \mathrm{m}^{2}$ condition. The error bars indicate the standard error. 
The most consistent age effect was observed in PLR latency. The ANCOVA model revealed that in children from 6 to 9 years old, latency has a significant age effect $\left(F_{3,132}\right.$ $=6.68, p<0.001)$. As shown in Fig. 4-2b, PLR latency decreased significantly at all testing conditions from 6 to 9 years and stabilized thereafter. For example, the PLR latency decreased from $259.0 \pm 4.3 \mathrm{~ms}$ at 6 years old to $237.3 \pm 6.3 \mathrm{~ms}$ at 8 years old at stimulation condition of LA $8721.1 \mathrm{~cd} / \mathrm{m}^{2}$. The ANOVA test for a linear trend in children younger than 10 years old further confirmed that the age effect was significant $(p<0.01)$ at all conditions except the one with the lowest stimulus intensity of LA $69.3 \mathrm{~cd} / \mathrm{m}^{2}$.

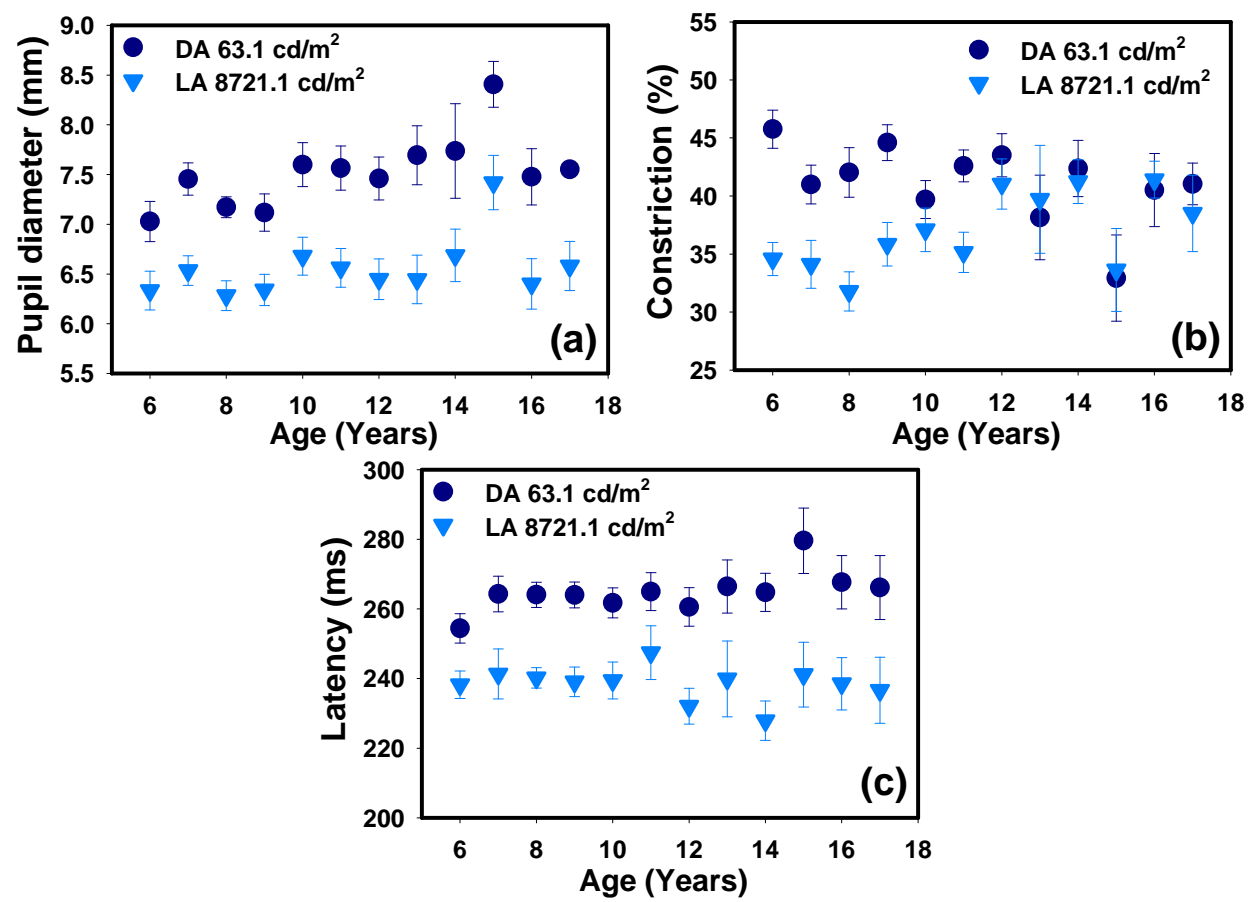

Fig. 4-3: PLR parameters obtained in children with neurodevelopment disorders from 6 to 17 years old.

(a) Resting pupil diameter, (b) Relative constriction amplitude and (c) latency measured in the light adapted (LA) $8721.1 \mathrm{~cd} / \mathrm{m}^{2}$ and dark adapted (DA) $63.1 \mathrm{~cd} / \mathrm{m}^{2}$ condition. The error bars indicate the standard error.

Since we saw a consistently significant age trend in PLR parameters (resting pupil 
diameter, constriction amplitude and latency), we examined age trends in the same PLR parameters in a group of children of the same age range with neurodevelopment disorders. As shown in Fig. 4-3, no age dependent trend in the resting pupil diameter, PLR constriction amplitude and PLR latency existed in this group of children. At the same stimulation condition, children with neurodevelopment disorders had significantly longer latency and significantly smaller constriction amplitude than typically developing children.

\subsection{Discussion}

PLR is an involuntary neurological response. Testing of PLR is noninvasive, simple and fast. It requires minimal cooperation from the subject and thus is convenient for testing in children. A good understanding of age dependent behavior of PLR is essential to evaluate the potential use of PLR for screening neurodevelopmental disorders in children.

Our results indicated a consistent and statistically significant age effect in PLR latency measured in young children ( $<10$ years old) with typical development. These results appear to be consistent with previous findings of age-dependent changes in visual evoked potential (VEP) in children. Lenassi et al, (2008) compared flash VEP and pattern VEP in infants and young children from 1.5 months to 7.5 years of age. They found that VEP latency for all three stimulation types showed an exponential decrease with age, but the trends were different. The latencies of reversal and pattern onset VEP showed fast decays (exponential decay rate of $-9.3 /$ year and $-13 /$ year respectively) and 
were stabilized by 6 months of age. However, flash VEP latency showed a slower decay (exponential decay rate of $-0.54 /$ year) and still decreased gradually at the upper limit of the age ( 7.5 years) they tested. Our age-dependent PLR latency in children ( $6-9$ years) with typical development had a similar effect as the flash VEP latency results reported by Lenassi et al. (2008). Carrillo-De-La-Peña et al. (1999) studied flash VEP in 85 children from 8 -15 years old and reported no significant age effect in latency. This result is consistent with our observation that PLR latency didn't change in children older than 9 years old.

The latency age trend we observed is similar to the age trend reported by Bashat et al. (2005) in high b value diffusion weight magnetic resonance imaging (DWI) study in a healthy sample aged 4 months to 23 years. Bashat et al. (2005) reported that, apparent-displacement decreases with age exponentially while stabilizing around 10 years of age. Bashat et al. (2005) also reported that, the fractional anisotropy (FA) and apparent-probability increases with age exponentially and stabilized around 10 years of age. FA measures the anisotropy, and increases with anisotropy. Due to the cylindrical geometry of the neuronal fibers in white matter, anisotropy in white matter is high compared to grey matter. Hence, FA increases with white matter maturation. Apparentdisplacement and apparent-probability measures restricted diffusion, with a major contribution from the intra-axonal water. With increased restriction, apparentdisplacement decreases while apparent-probability increases. When new neuronal connections establish with brain maturation, water content decreases and surface membranes, lipids and proteins increase, thus increasing restriction. Therefore, with 
new neuronal connection establishment, apparent-displacement decreases and apparent-probability increases. The PLR latency trend observed, following the same age trend as these three parameters suggest, that myelination and increased white matter organization might have an effect in terms of increasing the signal conduction speed, thus reducing the PLR latency in matured brain.

The resting pupil diameter increase observed in early ages of life (till 12 years) followed by a decrease after 12 years is similar to results previously reported in dark adapted pupil diameter (Kohnen et al., 2004). Immaturity of the brain causing low mental and emotional activity and sympathetic discharges and central inhibition of the parasympathetic sphincter might be the reason for small pupil diameters observed in small children (Loewenfeld, 1999). Winn et al. (1994) showed that in light adapted conditions, age dependency in pupil size appears to be reduced.

Although a significant age effect was reported in relative constriction, it was statistically significant only at one test condition with the highest stimulus intensity. With a close examination, we noticed that the coefficient of variance for relative constrictions varied from $12 \%$ to $63 \%$ at those stimulation conditions where the age effect was not statistically significant. At the strongest stimulus of LA at $8721.1 \mathrm{~cd} / \mathrm{m}^{2}$, the coefficient of variance was much smaller, from $8 \%$ to $24 \%$. Hence it is possible that the lack of statistical significance can be attributed to the higher variation in data obtained with smaller stimulus intensities.

The fact that no age-dependent trend in PLR latency or resting pupil diameter was observed in the group of children with neurodevelopment disorders suggests that 
the typical neurodevelopmental trajectory might be altered in neurodevelopmental disorders. The underlying mechanisms need further study. However, our result suggests that PLR has the potential to provide clinically useful information about progression of neural development in children.

\subsection{Conclusion}

We found a significant and consistent age dependent effect in PLR latency in children 6 to 9 years old. We also observed age effects in resting pupil diameter and PLR constriction amplitude. Such an age-dependent effect was not observed in children with neurodevelopment disorders. Further studies in larger groups of children especially in children younger than 6 years old are necessary to fully understand the details of age

dependency of PLR. Nevertheless, PLR shows potential to be applied as a simple noninvasive tool to monitor neurodevelopment in children. 


\section{CHAPTER 5}

\section{ATYPICAL PUPILLARY LIGHT REFLEX AND HEART RATE}

\section{VARIABILITY IN CHILDREN WITH AUTISM SPECTRUM}

\section{DISORDERS}

\subsection{Introduction}

Autism spectrum disorders (ASDs) are complex developmental disorders with symptoms in three core areas: social functioning, communication, and restricted or repetitive behaviors. While much progress has been made regarding ASD, the understanding of its etiology is still evolving (Geschwind and Levitt, 2007). Although diagnosis of ASD is based on behavioral assessment, various physical measures have also been used to look for the neurological dysfunctions underlying ASD. Among various measures, pupillary response has been an interesting target. Pupil size is controlled by two antagonistic iris muscles: the sphincter and the dilator (Barbur, 2004) and can be easily measured using non-invasive imaging methods. Pupillary responses can reveal a rich set of neurological information (Loewenfeld, 1999) and have long been used in both medical practice (Bremner, 2009) and psychophysical studies (Laeng et al., 2012).

A few studies compared baseline pupil size in children with ASD and typically

\footnotetext{
${ }^{*}$ The material presented in this chapter was published in Journal of Autism and Developmental Disorders. Daluwatte, C., Miles, J. H., Christ, S. E., Beversdorf, D. Q., Takahashi, T. N. \& Yao, G. 2012. Atypical Pupillary Light Reflex and Heart Rate Variability in Children with Autism Spectrum Disorder. Journal of Autism and Developmental Disorders, Dec 18. [Epub ahead of print]. 10.1007/s10803-012-1741-3
} 
developing children, but the results have been inconsistent. Anderson and Colombo (2009) found baseline pupil size was significantly larger in children with ASD than either mental age or chronological age matched controls when they were presented with grey slides. This finding was later replicated in two different samples of children with ASD (Anderson et al., 2012). However, Martineau et al. (2011) showed that children with ASD had significantly smaller baseline pupil size than typically developing children in response to a black slide. No difference in baseline pupil size was observed in a study by van Engeland et al. (1991) between the ASD group and typical controls. Fan et al. (2009a) also reported similar baseline pupil size in children with ASD and typically developing children in both dark- and light-adapted conditions, although the data variation was significantly higher in the ASD group.

It is recognized in clinical tests (Bremner, 2009) that resting pupil size may vary over a wide range even in individuals without any medical problems. On the other hand, the dynamic changes in pupil size induced by various stimuli may provide more reliable information about the neurological system (Bremner, 2009). Anderson et al. (2006) reported an atypical pupillary response in children with ASD when viewing children's faces. Specifically, the ASD group showed pupillary constriction in response to children's faces; whereas children with typical development or developmental delays (non-ASD) showed pupil dilation. Martineau et al. (2011) revealed that the pupillary responses to neutral faces, virtual faces, and objects followed a similar three-phase time course in both children with ASD and typical controls, i.e. a rapid initial dilation followed with a rapid constriction and then a slow recovery to baseline. Recently, Wagner et al. (2012) 
reported that pupillary response to emotional faces was similar in adolescents with ASD and typical controls.

In comparison to the aforementioned social stimuli, luminance change is an easier way to induce consistent pupillary responses (Barbur, 2004). When stimulated by a flash of light, the pupil undergoes a characteristic process to constrict and then recover (Bremner, 2009), which is referred to as pupillary light reflex (PLR). Atypical PLR was previously reported in children with ASD (Rubin, 1961; Fan et al., 2009a). Rubin (1961) discovered that the pupillary constriction speed $\left(V_{c}\right)$ was significantly slower in children with autism than typical controls when stimulated using a constant light intensity. This observation was confirmed by Fan et al. (2009a) using a short $100 \mathrm{~ms}$ optical stimulus at several different intensities. Fan et al. (2009a) also reported a significantly longer PLR latency and reduced constriction amplitude associated with ASD.

The PLR pathway includes the retina, pretectal olivary nucleus, Edinger-Westphal nucleus, and ciliary ganglion (Lowenstein and Loewenfeld, 1950; Appenzeller, 1999). This PLR pathway is largely under the influence of the parasympathetic pathway of the autonomic nervous system (ANS) (Neuhuber and Schrödl, 2011). Parasympathetic preganglionic neuron which originate in the Edinger-Westphal nucleus, synapses at the ciliary ganglion, control the sphincter muscle. Sympathetic long ciliary nerves from the superior cervical ganglion control the dilator muscle which may also modulate the pupillary dilation process. As a result, PLR parameters can be influenced by ANS dysfunction (Bremner, 2009).

ANS dysfunction has been reported in children with ASD in several studies. Ming 
et al. (2011) reported that families endorsed significantly more symptoms of autonomic dysfunction in their children with ASD than control families. Several studies have reported elevated heart rate in individuals with ASD in comparison to typically developing controls (Kootz and Cohen, 1981; Ming et al., 2005; Bal et al., 2010). Ming et al. (2005) also found higher mean arterial and diastolic blood pressure, lower cardiac vagal tone and lower cardiac sensitivity to baroreflex in children with ASD. These findings suggest that children with ASD have an elevated autonomic arousal. In addition, lower baseline respiratory sinus arrhythmia was reported in children with ASD (Bal et al., 2010) suggesting a reduced vagal modulation in ASD. However, Mathewson et al. (2011) demonstrated that baseline cardiac autonomic measures were significantly affected by medication use in adults with ASD.

Heart rate and heart rate variability (HRV), which measures the beat-to-beat variations of the heart rate, are regulated by the ANS. Vagal activity reduces heart rate through the sinoatrial (SA) and atrioventricular (AV) nodes, while sympathetic activation increases the heart rate also through the SA node. HRV parameters are considered an objective assessment of cardiac autonomic function (Kamath and Fallen, 1993; Thayer and Sternberg, 2006). HRV has been used to evaluate ANS dysfunction in disorders such as panic disorder (Yeragani et al., 1993), schizophrenia (Bär et al., 2007), and sleep disorders (Bonnet and Arand, 1998). Interestingly, a significant correlation between HRV and PLR was previously reported in patients with acute schizophrenia (Bär et al., 2008). However, HRV has not been investigated extensively in ASD.

The purpose of this present study is to investigate the atypical PLR associated 
with ASD in a larger heterogeneous sample. To study the potential association between atypical PLR and other ANS dysfunction in children with ASD, we simultaneously measured HRV during the PLR test. Because of the involvement of cognitive impairment and medication taking in children with ASD, their potential effects on PLR and HRV parameters were studied. We also tested a group of children with non-ASD neurodevelopmental disorders to investigate whether atypical PLR is specific to ASD. Due to the wide age distribution in the test population, the potential age effects on PLR and HRV parameters were also examined.

\subsection{Methods}

\subsubsection{Participants}

A total of 152 children with an ASD participated in this study (referred to as the "ASD" group). The ages ranged from 5 to 19 years with an average age of $10.7 \pm 3.4$ years; the group consisted of 135 boys ( $10.9 \pm 3.5$ years) and 17 girls ( $9.8 \pm 2.6$ years). Of the 152 participants, 145 were patients receiving clinical services at the University of Missouri Thompson Center for Autism and Neurodevelopmental Disorders, an interdisciplinary academic medical center specializing in diagnosis and treatment of ASD. Diagnostic interviews, caregiver questionnaires, and observation focusing on DSMIV criteria (American Psychiatric Association, 2000) were used for the diagnosis of ASD in these individuals. The Autism Diagnostic Observation Schedule (ADOS) (Lord et al., 1989) was obtained for 112 participants and the Autism Diagnostic Interview - Revised (ADI-R) (Lord et al., 1994) was obtained for 80 patients; the ASD diagnosis was 
confirmed in all of these cases. Evaluations were conducted by a pediatrician and/or neuropsychologist; if there was disagreement, the results were discussed jointly to reach a consensus diagnosis. The remaining 7 children were diagnosed using a variety of measures, which were reviewed by the authors to confirm the ASD diagnosis. In addition, each of these 7 families completed the Social Communication Questionnaire Lifetime (SCQ) (Eaves et al., 2006) and Social Responsive Scale Questionnaire (SRS) (Constantino and Gruber, 2005), all of which were scored above the ASD cutoff.

Among the 152 children with ASD, 86 were diagnosed with classic autism (autistic disorder), 32 with Asperger's Syndrome, and 34 with pervasive developmental disorder-not otherwise specified (PDD-NOS). Seventy children in the ASD group had taken one or more medications (includes stimulants, atypical antipsychotics, serotonin reuptake inhibitors, antihistamines, antiepileptics, etc.) within 48 hours before the PLR test (referred to as the "w/med" group). The remaining children had not taken medication (referred to as the "w/o med" group).

A sample of 116 typically developing healthy children between 6 and 17 years of age without known visual, neurological, or cardiovascular problems comprised a typically developing comparison group (referred to as the "TD" group). Nine children who had a sibling with ASD were excluded from the data analysis. Thus, 107 children (mean age $=10.9 \pm 2.9$ years) were included in the TD group, which consisted of 79 boys (mean age $=11.1 \pm 3.1$ years) and 28 girls (mean age $=10.6 \pm 2.4$ years). All participants in the TD group scored below the clinical cutoff $(<15)$ on the Social Communication Questionnaire Lifetime (Eaves et al., 2006) (mean score $=2.3 \pm 2.8$ ). None of the TD 
participants had taken medications within 48 hours before the PLR test.

A sample of 36 children ranging in age from 5 to 17 years of age (mean age $=9.9$ \pm 3.0 years) with intellectual disabilities due to other neurodevelopmental disorders (NDDs) also participated in this study. This group, referred to as the "NDD" group, included 27 boys (mean age $=10.0 \pm 3.1$ years) and 9 girls (mean age $=9.7 \pm 2.6$ years) This group included Down syndrome (7), Fragile X syndrome (5), Neurofibromatosis Type One (1), Prader-Willi syndrome (1), and the remainder with idiopathic intellectual impairment. All participants in this group were assessed to confirm that they did not meet the diagnostic criteria for ASD. Nineteen children in the NDD group were on medications similar to those described above for the ASD group.

Intelligence quotient (IQ) scores were available for all participants with the exception of 30 children in the ASD group, 7 in the TD group and 2 in the NDD group. The vast majority of IQ scores were derived from the Ravens Progressive Matrices (RPM) (Raven et al., 1996) ( $n=81$ ASD, 100 TD, and 34 NDD). The remainder were derived from the Wechsler Abbreviated Test of Intelligence ( $n=12$ ASD), Differential Abilities Scale $2^{\text {nd }}$ Edition ( $n=15$ ASD), Leiter International Performance Scale - Revised ( $n=9$ ASD) and Stanford-Binet Intelligence Scales - Fifth Edition ( $n=5$ ASD). For purposes of later analysis of the relationship between overall intellectual ability and PLR parameters, participants were categorized into either the "Low IQ" group or the "High IQ" group. An IQ equivalent of 80 or higher (9.1 percentile) was used to designate a child with normalto-above normal intelligence (Wechsler, 1991). Thus, the 9.1 percentile was used for those who were assessed with the RPM, and a threshold score of 80 was used for 
children who had been assessed by other IQ tests. Distributions of the IQ subgroups and medication status of participants are shown in Table 5-1.

Table 5-1: Distribution of IQ and medication use in TD, ASD and NDD groups.

\begin{tabular}{|c|c|c|c|c|}
\hline & & IQ & w/o med & $\mathrm{w} / \mathrm{med}$ \\
\hline TD & & High-IQ & 98 & 0 \\
\hline & & Low-IQ & 2 & 0 \\
\hline 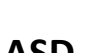 & & High-IQ & 44 & 34 \\
\hline ADD & & Low-IQ & 23 & 21 \\
\hline & Acnorgar & High-IQ & 9 & 11 \\
\hline 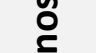 & Aspeiget & Low-IQ & 2 & 0 \\
\hline 垔 & Autism & High-IQ & 23 & 14 \\
\hline$\overline{0}$ & " & Low-IQ & 19 & 15 \\
\hline & PDD-NOS & High-IQ & 12 & 9 \\
\hline & & Low-IQ & 2 & 6 \\
\hline NDD & & High-IQ & 9 & 9 \\
\hline & & Low-IQ & 7 & 9 \\
\hline
\end{tabular}

High-IQ: IQ score of 80 or higher (at or above $9.1^{\text {th }}$ percentile)

Low-IQ: IQ score lower than 80 (below $9.1^{\text {th }}$ percentile)

This study was approved by the Institutional Review Board of the University of Missouri. All participants and their legal guardians provided written informed assent and consent prior to participating.

\subsubsection{PLR Instrument}

The binocular pupillography recording system used in this study is similar to that described previously (Fan et al., 2009a, 2009b). The system uses near-infrared imaging cameras (GC660, Allied Vision Technologies, Stadtroda, Germany) to record pupil images at a speed of 115 frames-per-second (fps). The spatial resolution of the imaging system is $0.035 \mathrm{~mm} /$ pixel. A $100 \mathrm{~ms}$ optical stimulus is produced using $530 \mathrm{~nm}$ green LEDs 
which illuminates a circular optical diffuser. The illuminated diffuser is positioned at 12.5 $\mathrm{cm}$ from the eye and has an effective diameter of $1.27 \mathrm{~cm}$ (an equivalent visual field of $\left.5.7^{\circ}\right)$. The stimulus intensity was controlled by adjusting the electric current to the LED and by using different neutral density filters.

To obtain heart rate variability (HRV) in our population, the heart beat signal (RR tachogram) was recorded using a wireless heart rate measuring device (Polar RS800CX, Polar Electro Oy, Finland). A chest strap with an enclosed heart rate sensor measured the QRS intervals at a rate of $1 \mathrm{kHz}$. Several studies have found that the performance of this device is consistent with the conventional 12-lead ECG system (Gamelin et al., 2008; Goodie et al., 2000; Nunan et al., 2009; Porto and Junqueira Jr, 2009).

\subsubsection{Test Procedure}

The PLR test procedure was performed as described in detail previously (Daluwatte et al., 2012a). In brief, throughout testing the child was seated in a comfortable chair with a back. Heart rate measurements were begun 5 minutes prior to the PLR testing and continued for 5 minutes following completion of the PLR testing. Participants fixed the sight on pictures of animals or toys displayed on a dim computer monitor placed $1.3 \mathrm{~m}$ away from the eye. PLR was first measured in light adapted (LA) conditions (220 Ix room luminance) using 3 different stimulus intensities in ascending order: LA $69.3 \mathrm{~cd} / \mathrm{m}^{2}$, LA $872.1 \mathrm{~cd} / \mathrm{m}^{2}$, and LA $8721.1 \mathrm{~cd} / \mathrm{m}^{2}$. The dark-adapted (DA) PLR was then measured at a stimulation intensity of DA $63.1 \mathrm{~cd} / \mathrm{m}^{2}$ after $15-\mathrm{min}$ of dark adaptation $(<0.01 \mathrm{Ix}$ room luminance). For each stimulus condition, the left eye was 
stimulated 4 times and then the right eye was stimulated 4 times. A 30-sec interval was provided between consecutive stimulations. We tested $43 \%$ of the participants in the ASD group, $36 \%$ in the TD group, and $53 \%$ in the NDD group in the morning, while the remaining was tested in the afternoon.

\subsubsection{Data Analysis}

The pupilogram (Fig. 1-2) was constructed by extracting the pupil size from acquired pupil images as described in detail elsewhere (Fan et al., 2009a). The following PLR parameters were calculated from the pupilogram to quantify the child's pupillary response (Fig. 1-2): (1) the baseline pupil diameter $\left(D_{0}\right)$, defined as the average resting pupil diameter before stimulus onset; (2) the relative constriction amplitude, calculated as $A_{\%}=\left(D_{0}^{2}-D_{m}^{2}\right) / D_{0}^{2}$, where $D_{m}$ is the minimal pupil diameter during constriction; (3) the latency $\left(t_{L}\right)$, defined as the time that elapsed between stimulus onset and the beginning of pupil constriction; $(4)$ the constriction time $\left(t_{c}\right)$, defined as the time interval between the beginning of pupil constriction and when pupil reached minimal diameter $D_{m} ;(5)$ the redilation time $\left(t_{R}\right)$, calculated as the time interval between the minimal diameter $D_{m}$ and when the pupil recovered to half of the constriction; (6) the constriction velocity $\left(\mathrm{v}_{\mathrm{C}}\right)$, calculated as $\left(D_{0}-D_{m}\right) / 2 \mathrm{t}_{C}$; and $(7)$ the redilation velocity $\left(\mathrm{v}_{\mathrm{R}}\right)$, calculated as $\left(D_{0}-D_{m}\right) / 4 \mathrm{t}_{\mathrm{R}}$. PLR data from both eyes obtained during 8 repeated measurements were averaged to calculate the mean value and standard deviation at each stimulus condition. PLR images of 2 children in the ASD group, 1 child in the TD 
group, and 3 children in the NDD group could not be processed because of excessive eye movement or closure during the test.

In addition to the average heart rate (AHR), heart rate variability (HRV) was calculated using both time-domain and frequency-domain analyses as explained by Malik et al. (1996). Two time-domain parameters were calculated: (1) the standard deviation of normal to normal (NN) intervals (SDNN) and (2) the root mean square of successive differences (rMSSD). The frequency-domain power spectrum was analyzed using fast Fourier transform (FFT). Two frequency-domain HRV parameters were calculated: the normalized power of the high-frequency band $\left(\mathrm{HF}_{\mathrm{N}}\right)(\mathrm{HF}=0.15-0.4 \mathrm{~Hz})$ and the LF/HF power ratio, where the low-frequency bandwidth was $0.04-0.15 \mathrm{~Hz}$. $\mathrm{HF}_{\mathrm{N}}$ is generally considered as an indicator of vagal activity and is correlated with rMSSD (Malik et al., 1996). SDNN carries influences from both parasympathetic and sympathetic modulation (Malik et al., 1996). The LF/HF ratio may reflect the "sympathetic outflow" or the "sympathovagal balance" (Malik et al., 1996; Berntson et al., 1997).

To determine any potential effect of participating in the PLR procedure on heart rate variability, the HRV was analyzed in the following 5 different "HRV measurement phases": (1) before the PLR test (5 min), (2) during LA PLR (10 min), (3) during dark adaptation (15 min), (4) during DA PLR (5 min), and (5) after the PLR test (5 min). We were not able to acquire HRV in 9 children in the ASD group, 1 in the TD group, and 1 in the NDD group because the participants declined to wear the heart rate sensor. A 
malfunction of the heart rate sensor resulted in missing HRV data in 2 other children in the ASD group.

The Kolmogorov-Smirnov test was used to verify normal distributions of all measured PLR and HRV parameters. For each PLR and HRV parameter, the Analysis of Covariance (ANCOVA) using the PROC MIXED procedure in SAS (Version 9.2, SAS Institute Inc., Cary, NC, USA) was applied to examine the effects of group (TD, ASD, and NDD), age, and test conditions (stimulus intensity/HRV measurement phase and time of day of the test). Follow up analysis of variance (ANOVA), multivariate analysis of variance (MANOVA), and t-tests with Bonferroni correction were used appropriately to confirm effects revealed by the ANCOVA model. ANOVA model was applied to study the effects of IQ (High IQ and Low IQ) and medication ("w/o med" and "w/med") in the ASD and NDD groups, and the effect of ASD diagnosis (classic autism, Asperger's, and PDDNOS) in the ASD group. The method reported by Steyn and Ellis (2009) was applied to

evaluate effect size $\left(\hat{\eta}_{\Lambda, r=1}^{2}\right)$ for group differences using MANOVA. An $\hat{\eta}_{\Lambda, r=1}^{2}$ value of $0.02,0.13$ and 0.26 was considered as a small, medium and large effect, respectively (Steyn and Ellis, 2009). Pearson product moment correlation was applied to study correlation between PLR parameters and HRV parameters. A $p$ value $<0.05$ was considered significant.

\subsection{Results}

The mean and standard deviations of all measured PLR and HRV parameters in the TD, ASD, and NDD groups are shown in Table 5-2 and Table 5-3, respectively. 
Table 5-2: Summary of PLR results.

\begin{tabular}{|c|c|c|c|c|c|c|c|}
\hline $\begin{array}{l}\text { Stimulus } \\
\text { intensity } \\
\left(\mathrm{cd} / \mathrm{m}^{2}\right)\end{array}$ & $\begin{array}{l}\text { Resting } \\
\text { pupil } \\
\text { diameter } \\
(\mathrm{mm})\end{array}$ & $\begin{array}{l}\text { PLR latency } \\
\text { (ms)* }\end{array}$ & $\begin{array}{l}\text { Constricti } \\
\text { on (\%)* }\end{array}$ & $\begin{array}{l}\text { Constriction } \\
\text { time }(\mathrm{ms})^{*}\end{array}$ & $\begin{array}{l}\text { Redilation } \\
\text { time (ms)* }\end{array}$ & $\begin{array}{c}\text { Constrictio } \\
\mathrm{n} \text { velocity } \\
\text { (mm/s) }\end{array}$ & $\begin{array}{c}\text { Redilation } \\
\text { velocity } \\
(\mathrm{mm} / \mathrm{s})\end{array}$ \\
\hline \multicolumn{8}{|l|}{$\overline{T D}$} \\
\hline LA 69.3 & \multirow{3}{*}{$6.58 \pm 0.61$} & $274.3 \pm 23.9$ & $11.8 \pm 5.5$ & $370.7 \pm 73.4$ & $402.0 \pm 86.3$ & $0.81 \pm 0.44$ & $0.37 \pm 0.20$ \\
\hline LA 872.1 & & $239.0 \pm 16.2$ & $26.9 \pm 7.1$ & $399.2 \pm 52.8$ & $498.1 \pm 99.9$ & $1.75 \pm 0.75$ & $0.72 \pm 0.30$ \\
\hline LA 8721.1 & & $214.4 \pm 14.4$ & $40.8 \pm 7.2$ & $464.5 \pm 51.2$ & $595.6 \pm 116.6$ & $2.37 \pm 0.95$ & $0.98 \pm 0.45$ \\
\hline DA 63.1 & $7.44 \pm 0.77$ & $244.0 \pm 15.4$ & $44.7 \pm 6.9$ & $580.2 \pm 59.4$ & $804.4 \pm 171.8$ & $2.41 \pm 0.97$ & $0.91 \pm 0.36$ \\
\hline \multicolumn{8}{|c|}{ e } \\
\hline LA 69.3 & \multirow{3}{*}{$6.50 \pm 0.81$} & $302.2 \pm 32.2$ & $9.6 \pm 6.1$ & $336.7 \pm 75.6$ & $370.3 \pm 89.2$ & $0.76 \pm 0.50$ & $0.35 \pm 0.21$ \\
\hline LA 872.1 & & $265.3 \pm 25.4$ & $22.5 \pm 8.3$ & $364.5 \pm 63.4$ & $455.8 \pm 104.0$ & $1.65 \pm 0.76$ & $0.68 \pm 0.31$ \\
\hline LA 8721.1 & & $237.7 \pm 22.5$ & $36.6 \pm 8.5$ & $434.1 \pm 62.9$ & $560.7 \pm 106.1$ & $2.39 \pm 0.91$ & $0.95 \pm 0.35$ \\
\hline DA 63.1 & $7.47 \pm 0.88$ & $262.7 \pm 18.5$ & $41.6 \pm 7.7$ & $552.1 \pm 73.8$ & $737.8 \pm 155.0$ & $2.55 \pm 0.91$ & $0.99 \pm 0.40$ \\
\hline \multicolumn{8}{|l|}{ NDD } \\
\hline LA 69.3 & \multirow{3}{*}{$6.36 \pm 0.74$} & $307.1 \pm 44.6$ & $10.5 \pm 5.8$ & $372.9 \pm 77.1$ & $415.7 \pm 117.4$ & $0.65 \pm 0.46$ & $0.29 \pm 0.19$ \\
\hline LA 872.1 & & $270.9 \pm 22.1$ & $22.1 \pm 7.3$ & $379.4 \pm 60.1$ & $475.5 \pm 86.8$ & $1.42 \pm 0.86$ & $0.55 \pm 0.34$ \\
\hline LA 8721.1 & & $245.6 \pm 23.6$ & $36.5 \pm 8.8$ & $446.0 \pm 66.8$ & $562.2 \pm 85.6$ & $1.99 \pm 1.01$ & $0.78 \pm 0.34$ \\
\hline DA 63.1 & $7.14 \pm 0.83$ & $269.9 \pm 26.2$ & $42.2 \pm 8.5$ & $566.3 \pm 107.4$ & $730.0 \pm 116.5$ & $2.00 \pm 0.85$ & $0.79 \pm 0.35$ \\
\hline
\end{tabular}

The results are represented as group mean \pm standard deviation.

* Significant group difference (ANCOVA $p<0.0001$ ).

Table 5-3: Summary of HRV results.

\begin{tabular}{ccccccc}
\hline & $\begin{array}{c}\text { HRV measurement } \\
\text { phase }\end{array}$ & AHR (bmp) & SDNN (ms) & rMSSD (ms) & LF/HF (n.u.) & HF $_{\mathbf{N}}$ (\%) \\
\cline { 2 - 7 } & $\mathbf{1}$ & $90.1 \pm 12.4$ & $64.2 \pm 24.3$ & $38.2 \pm 20.5$ & $2.7 \pm 1.6$ & $31.2 \pm 10.3$ \\
\multirow{4}{*}{ TD } & $\mathbf{2}$ & $90.0 \pm 12.1$ & $69.7 \pm 24.3$ & $36.9 \pm 17.7$ & $4.5 \pm 5.2$ & $22.8 \pm 8.0$ \\
& $\mathbf{3}$ & $93.1 \pm 12.5$ & $66.4 \pm 28.0$ & $32.6 \pm 17.1$ & $3.4 \pm 1.6$ & $26.3 \pm 10.0$ \\
& $\mathbf{4}$ & $91.0 \pm 13.0$ & $72.0 \pm 26.3$ & $36.4 \pm 18.2$ & $4.4 \pm 3.0$ & $22.7 \pm 9.5$ \\
& $\mathbf{5}$ & $92.9 \pm 13.3$ & $65.4 \pm 27.2$ & $33.2 \pm 17.7$ & $4.1 \pm 0.3$ & $25.2 \pm 10.5$ \\
& $\mathbf{1}$ & $95.2 \pm 14.0$ & $56.9 \pm 20.1$ & $32.2 \pm 15.6$ & $2.9 \pm 1.8$ & $29.5 \pm 10.8$ \\
\multirow{4}{*}{ ASD } & $\mathbf{2}$ & $96.1 \pm 13.7$ & $61.1 \pm 20.9$ & $31.4 \pm 15.6$ & $3.7 \pm 2.1$ & $24.7 \pm 9.9$ \\
& $\mathbf{3}$ & $99.8 \pm 12.9$ & $56.8 \pm 23.4$ & $27.4 \pm 14.0$ & $3.4 \pm 1.7$ & $25.2 \pm 8.3$ \\
& $\mathbf{4}$ & $97.0 \pm 13.0$ & $60.6 \pm 23.8$ & $30.6 \pm 15.5$ & $3.7 \pm 1.9$ & $24.1 \pm 8.4$ \\
& $\mathbf{5}$ & $99.4 \pm 13.4$ & $57.1 \pm 23.3$ & $28.0 \pm 13.9$ & $3.8 \pm 2.6$ & $24.3 \pm 9.3$ \\
& $\mathbf{1}$ & $100.1 \pm 13.8$ & $51.4 \pm 18.3$ & $27.9 \pm 12.6$ & $2.4 \pm 1.2$ & $32.6 \pm 10.4$ \\
\multirow{4}{*}{ NDD } & $\mathbf{2}$ & $98.9 \pm 13.1$ & $54.6 \pm 18.2$ & $28.6 \pm 12.3$ & $3.1 \pm 1.6$ & $27.3 \pm 9.1$ \\
& $\mathbf{3}$ & $105.3 \pm 13.4$ & $44.8 \pm 13.6$ & $22.1 \pm 9.1$ & $3.0 \pm 1.3$ & $26.9 \pm 7.5$ \\
& $\mathbf{4}$ & $101.5 \pm 13.8$ & $51.5 \pm 19.2$ & $26.7 \pm 12.2$ & $3.7 \pm 2.0$ & $24.6 \pm 9.6$ \\
& $\mathbf{5}$ & $104.7 \pm 14.5$ & $45.9 \pm 15.4$ & $23.3 \pm 12.3$ & $3.5 \pm 1.8$ & $25.5 \pm 9.4$ \\
\hline
\end{tabular}

The results are represented as group mean \pm standard deviation.

* Significant group difference (ANCOVA $p<0.0001$ ).

bmp $=$ beats per minute

SDNN = standard deviation of normal to normal (NN) intervals

$\mathrm{rMSSD}=$ root mean square of successive differences

$\mathrm{LF}=$ low frequency

$\mathrm{HF}=$ high frequency 
The ANCOVA model revealed that the stimulation condition (adaptation and stimulus intensity) had a statistically significant effect $(p<0.0001)$ on all PLR parameters, including the constriction time $\left(t_{c}\right)$, relative constriction amplitude $\left(\Delta A_{\%}\right)$, latency $\left(t_{L}\right)$, redilation time $\left(t_{R}\right)$, constriction velocity $\left(v_{C}\right)$, and redilation velocity $\left(v_{R}\right)$. As expected, the resting pupil size was larger in DA than in LA. The PLR constriction amplitude, constriction time, and redilation time all increased with stimulus intensity, whereas the PLR latency decreased with stimulus intensity at the same adaptation. The constriction and redilation velocities also increased with stimulus intensity in LA and were larger in DA tests than LA tests at similar stimulus intensities. The interaction between group and stimulus was not significant for any of the PLR parameters, which suggests that the stimulus dependency was similar in all subject groups.

\subsubsection{Subject Group Differences}

\subsubsection{Group Differences in PLR Parameters}

The PLR parameters were significantly different between the TD and ASD groups, and between the TD and NDD groups, but not between the ASD and NDD groups.

The ANCOVA model indicated that the group (TD, ASD, and NDD) had a significant effect on PLR latency $\left(F_{2,1107}=150.44 p<0.0001\right)$, relative constriction amplitude $\left(F_{2,1106}=29.96 p<0.0001\right)$, constriction time $\left(F_{2,1103}=31.69 p<0.0001\right)$, and redilation time $\left(F_{2,1096}=14.67 p<0.0001\right)$. Post-hoc MANOVA confirmed that the ASD

and NDD groups had a significantly longer latency $\left(\mathrm{F}_{4,229}=23.24 p<0.0001 \hat{\eta}_{\Lambda, r=1}^{2}=0.28\right.$ 
for ASD; $\mathrm{F}_{4,130}=21.69 p<0.0001 \hat{\eta}_{\Lambda, r=1}^{2}=0.38$ for NDD) and lesser relative constriction amplitude $\left(\mathrm{F}_{4,231}=4.47 p=0.002 \hat{\eta}_{\Lambda, r=1}^{2}=0.06\right.$ for $\mathrm{ASD} ; \mathrm{F}_{4,130}=3.74 p=0.007 \hat{\eta}_{\Lambda, r=1}^{2}=0.08$ for NDD) than those of the TD group for all testing conditions. The ASD group also had a shorter constriction time (MANOVA $\mathrm{F}_{4,228}=5.01 p=0.0007 \hat{\eta}_{\Lambda, r=1}^{2}=0.06$ ) and redilation time (MANOVA $\mathrm{F}_{4,225}=3.39 p=0.01 \hat{\eta}_{\Lambda, r=1}^{2}=0.04$ ) than those of the TD group. The mean PLR latency of the NDD group appeared to be longer than that of the ASD group, but the difference was not statistically significant (MANOVA $F_{4,152}=1.71 p=0.15$ ). No significant group differences were found for other PLR parameters.

\subsubsection{Group Differences in AHR and HRV Parameters}

The ASD and NDD groups had significantly different AHR and HRV parameters than the TD group. The NDD group showed a significantly faster AHR than the ASD group.

The ANCOVA model revealed a significant group effect on AHR $\left(F_{2,1343}=50.81 p<\right.$ $0.0001)$ and on time-domain HRV parameters $\left(F_{2,1340}=41.92 p<0.0001\right.$; and $F_{2,1340}=$ $27.46 p<0.0001$ for SDNN and rMSSD, respectively). Post-hoc MANOVA confirmed that children with ASD had a significantly faster heart rate than that of typical controls in all 5 HRV measurement phases $\left(F_{5,218}=3.32 p=0.007 \hat{\eta}_{\Lambda, r=1}^{2}=0.05\right)$ (Table 5-3). The mean values of SDNN and rMSSD were lower in the ASD group than the TD group. However, MANOVA revealed that these differences were not statistically significant $\left(F_{5,217}=2.00 p\right.$ $=0.08 ;$ and $F_{5,217}=1.46 p=0.20$ for SDNN and $r M S S D$, respectively). The AHR of the NDD 
group was significantly faster than that of the ASD group (MANOVA $F_{5,146}=2.63 p=0.03$ $\left.\hat{\eta}_{\Lambda, r=1}^{2}=0.05\right)$. The NDD group also had a significantly faster $\operatorname{AHR}\left(F_{5,132}=5.41 p=0.0001\right.$ $\left.\hat{\eta}_{\Lambda, r=1}^{2}=0.14\right)$, lower $\operatorname{SDNN}\left(F_{5,131}=4.70 p=0.0006 \hat{\eta}_{\Lambda, r=1}^{2}=0.12\right)$ and lower $\operatorname{rMSSD}\left(F_{5,131}\right.$ $\left.=2.63 p=0.03 \hat{\eta}_{\Lambda, r=1}^{2}=0.06\right)$ than those of the TD group.

\subsubsection{Age Effect}

A significant age effect on PLR latency was observed in the TD group, but not in the ASD group. Both ASD and TD groups showed similar age trends for average heart rate and HRV parameters.

In the TD group, the PLR latency decreased from 6 to 8 years and reached a plateau thereafter (Fig. 5-1). One 16-year-old and two 13-year-olds in the TD group were identified as outliers on the regression line (PROC ROBUSTREG procedure in SAS); hence, their data were not included in the data shown in Fig. 5-1. In children 6 to 8 years of age, the ANCOVA model indicated that the Age*Group interaction was a significant factor on PLR latency $\left(\mathrm{F}_{4,265}=3.26 p=0.01\right)$, suggesting that PLR latency had different age profiles in the 3 subject groups. Analysis using the CONTRAST statement of the GLM procedure with matrix $[+1,0,-1]$ in SAS confirmed that latency decreased from 6 to 8 years in the TD group $\left(F_{1,21}=0.22 p=0.64 ; F_{1,21}=4.85 p=0.039 ; F_{1,21}=8.97 p=0.007\right.$; and $F_{1,21}=7.49 p=0.012$ for latency measured at $L A 69.3 \mathrm{~cd} / \mathrm{m}^{2}, L A 872.1 \mathrm{~cd} / \mathrm{m}^{2}, \mathrm{LA}$ $8721.1 \mathrm{~cd} / \mathrm{m}^{2}$, and DA $63.1 \mathrm{~cd} / \mathrm{m}^{2}$, respectively). However, this decreasing trend did not 
exist in the ASD group at any of the 4 stimulus intensities $\left(F_{1,36}=0.37 p=0.55 ; F_{1,35}=\right.$ $0.07 p=0.79 ; F_{1,37}=0.08 p=0.93 ;$ and $\left.F_{1,35}=0.06 p=0.81\right)$.
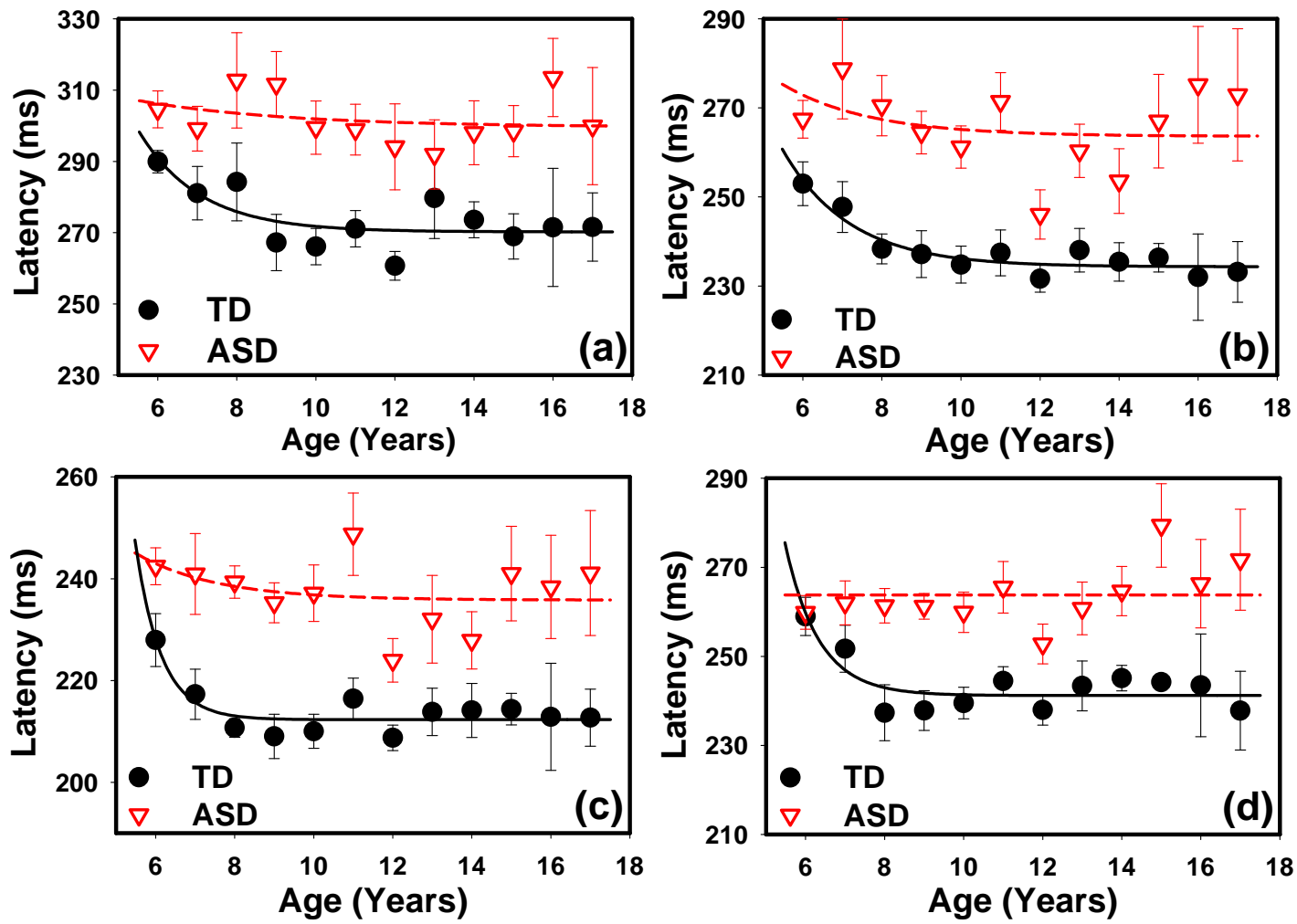

Fig. 5-1: PLR latency vs. age measured in the TD and ASD groups.

At different stimulus conditions: (a) LA $69.3 \mathrm{~cd} / \mathrm{m}^{2}$, (b) LA $872.1 \mathrm{~cd} / \mathrm{m}^{2}$, (c) LA 8721.1 $\mathrm{cd} / \mathrm{m}^{2}$, (d) $D A 63.1 \mathrm{~cd} / \mathrm{m}^{2}$. The lines are fitting results using an exponential decay function $\mathrm{y}=\mathrm{a}^{*} \exp \left(-\mathrm{b}^{*} \mathrm{x}\right)+\mathrm{c}$. The error bars indicate the standard error.

For further confirmation, the lines in Fig. 5-1 show the best curve fitting results using an exponential decay function $y=a \exp (-b x)+c$ with the curve-fitting tool in Matlab (Mathworks, MA). The TD results were well fitted with this function, with $\mathrm{R}^{2}$ ranging from 0.56 to 0.88 . However, either the ASD results could not be fitted with this exponential decay function or the decay was much slower than the TD results. The age effect was also not significant in the NDD group, although the number of participants was much smaller. 
The ANCOVA model revealed a significant age effect on AHR and both time- and frequency-domain HRV parameters. The $A H R, S D N N$, and $H F_{N}$ values measured during HRV measurement phase 1 (before the PLR test) in the TD and ASD groups are shown in Fig. 5-2. The AHR decreased with age in both groups. SDNN showed little change before 12 years of age but was increased in older children. $\mathrm{HF}_{\mathrm{N}}$ decreased with age in both the TD and ASD groups. Similar results were obtained in the other HRV measurement phases. A similar age effect on AHR was observed in the NDD group, but the time domain and the frequency domain parameters did not show a significant age effect in this group.
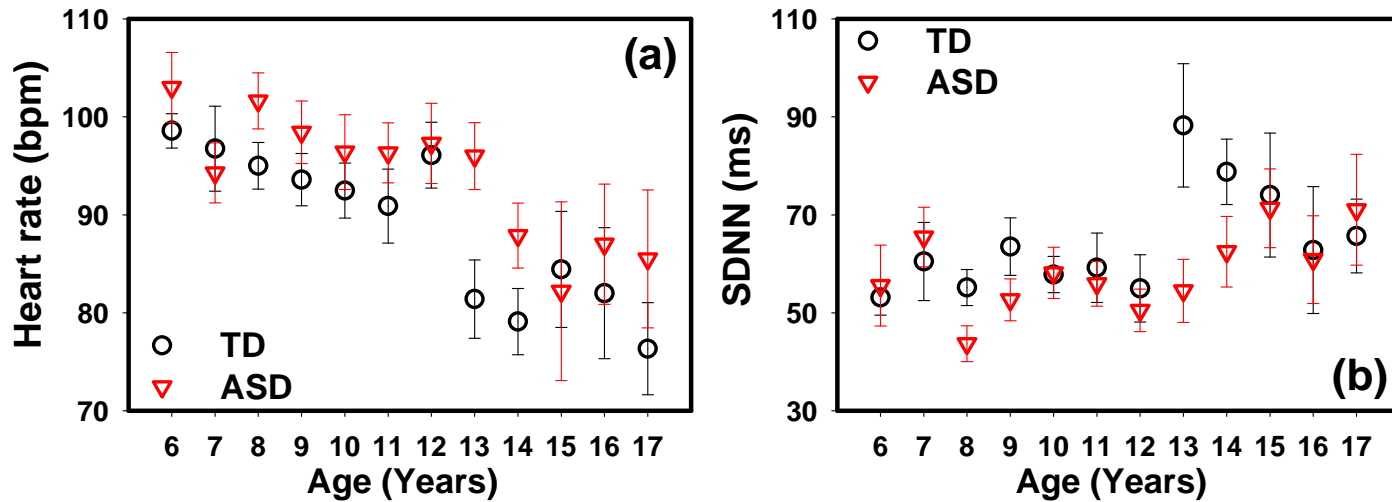

Age (Years)

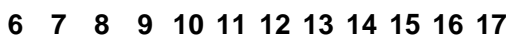
Age (Years)

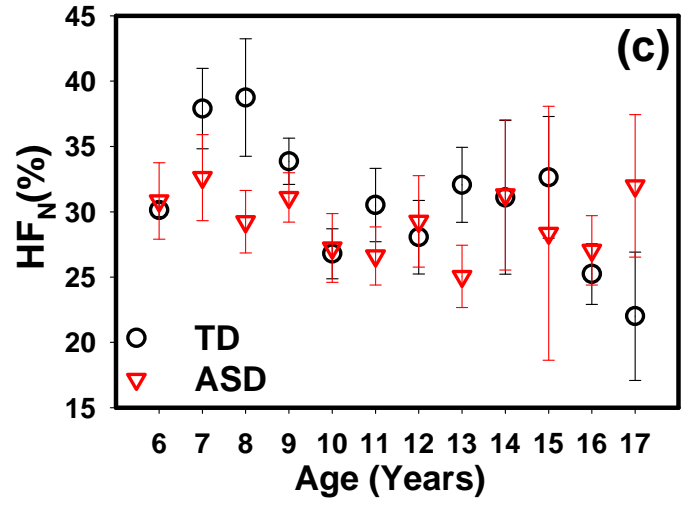

Fig. 5-2: The age effect in average heart rate, SDNN and $\mathrm{HF}_{\mathrm{N}}$. (a) average heart rate, (b) SDNN and (c) $\mathrm{HF}_{\mathrm{N}}$ obtained at different ages in the TD and ASD groups measured during the HRV measurement phase 1 (before PLR test). Similar results were obtained in other HRV measurement phases. The error bars indicate the standard error. 


\subsubsection{Medication Effect}

A medication effect was observed on average heart rate and HRV parameters, but not on PLR parameters.
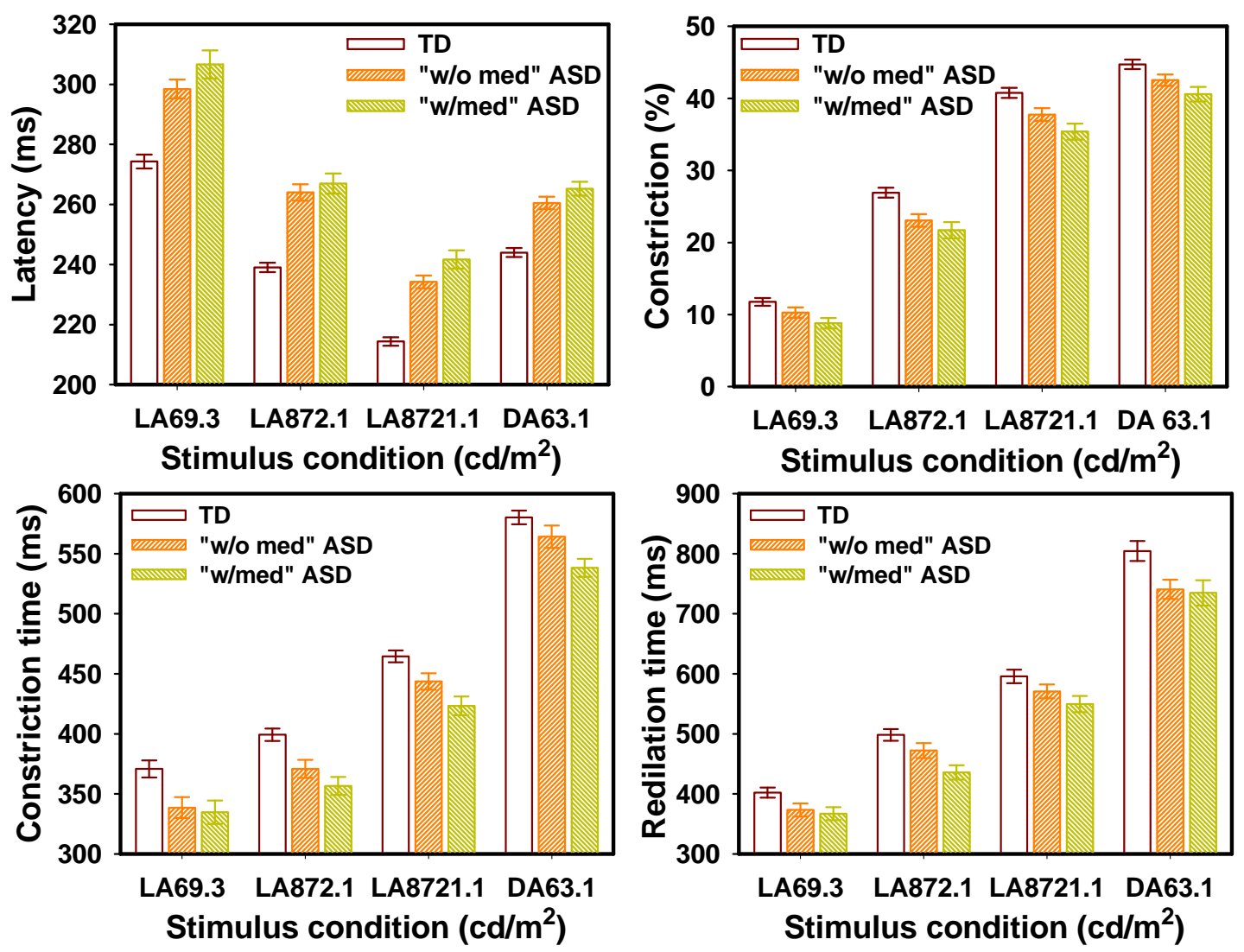

Fig. 5-3: Medication effect on PLR parameters.

PLR latency, constriction amplitude, constriction time and redilation time in the TD, "w/med" ASD, and "w/o med" ASD groups measured at different stimulus conditions. The error bars indicate the standard error.

The PLR latency in the TD group was significantly different from that in both the "w/med" ASD group $\left(\mathrm{F}_{4,159}=20.35 p<0.0001\right)$ and the "w/o med" ASD group $\left(\mathrm{F}_{4,171}=\right.$ $15.80 p<0.0001)$. The TD group also had significantly larger PLR constriction than both

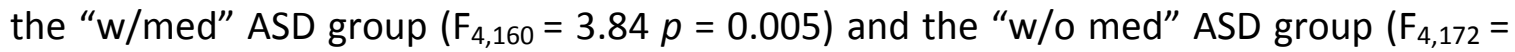
$2.90 p=0.023)$. Similarly, the PLR constriction time was significantly longer in the TD 
group than in both the "w/med" ASD group $\left(F_{4,159}=5.56 p=0.0003\right)$ and the "w/o med" ASD group $\left(F_{4,170}=2.77 p=0.029\right)$. Though the " $w /$ med" $^{\prime}$ ASD group appeared to have a slightly greater PLR latency, lesser constriction amplitude, and shorter constriction time than those of the "w/o med" ASD group (Fig. 5-3), the MANOVA indicated that these differences were not significant $\left(\mathrm{F}_{4,123}=0.92 p=0.45 ; \mathrm{F}_{4,125}=0.64 p=0.64 ;\right.$ and $\mathrm{F}_{4,122}=$ $1.25 p=0.29$ for latency, constriction amplitude, and constriction time, respectively). The redilation time was different only between the TD and " $\mathrm{w} / \mathrm{med}$ " ASD groups $\left(\mathrm{F}_{4,158}=\right.$ $3.63 p=0.007)$ but not between the TD and "w/o med" ASD group $\left(F_{4,168}=1.87 p=0.11\right)$ or between the "w/med" and "w/o med" ASD groups $\left(\mathrm{F}_{4,119}=0.96 p=0.43\right)$.
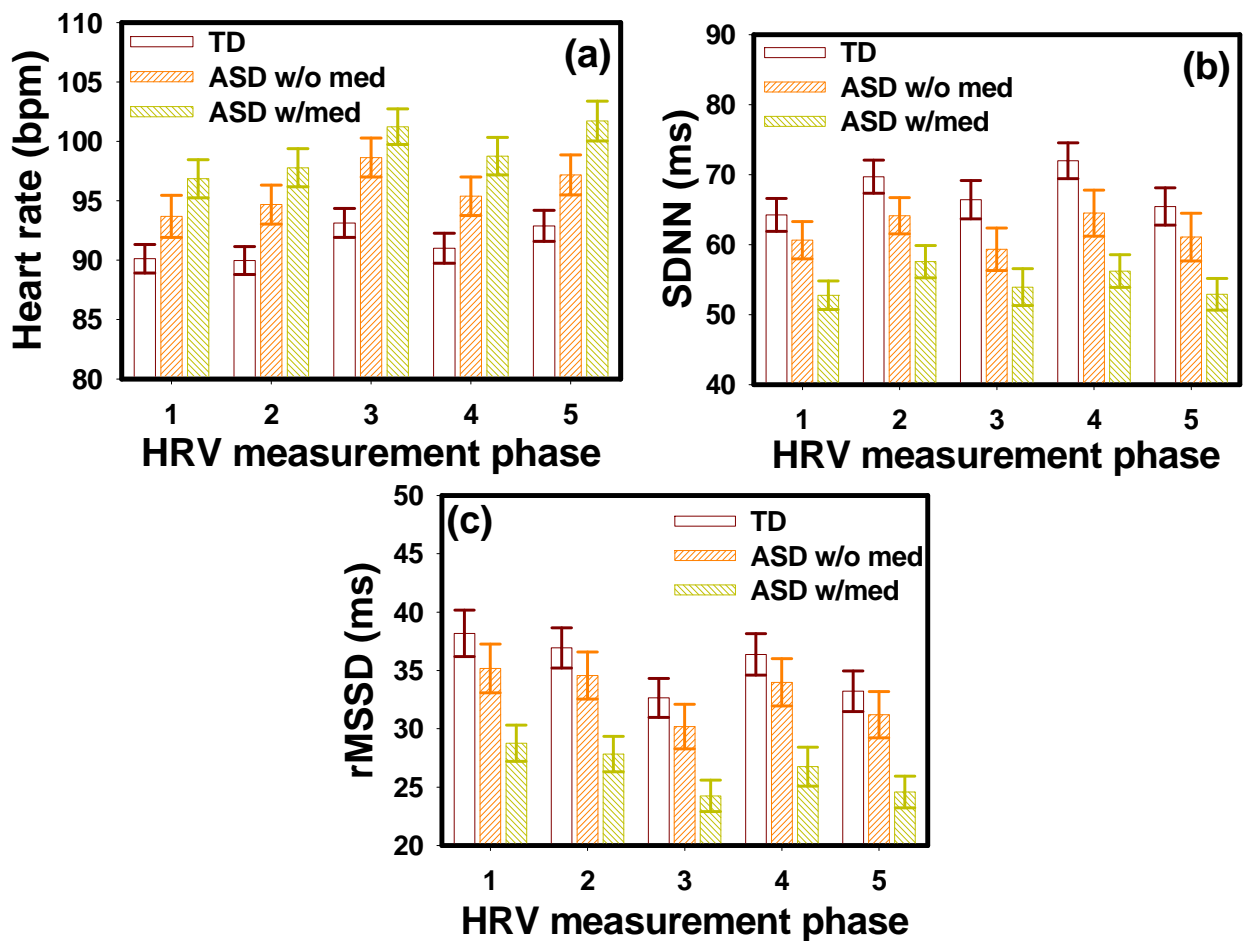

Fig. 5-4: Medication effect on average heart rate and HRV parameters.

The (a) average heart rate, (b) SDNN and (c) rMSSD in the TD, "w/med" ASD and "w/o med" ASD groups obtained in the five HRV measurement phases. The HRV measurement phases are numbered as 1: before PLR test, 2: during LA PLR, 3: during dark adaptation, 4: during DA PLR, and 5: after PLR test. The error bars indicate the standard error. 
The ASD "w/med" group had faster AHR and lesser SDNN and rMSSD than those of the ASD "w/o med" group (Fig. 5-4). The MANOVA test indicated significant group

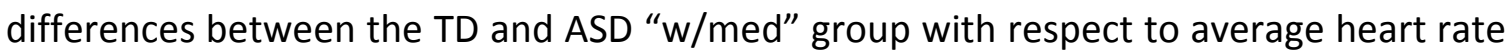
$\left(F_{5,157}=3.75 p=0.003\right), \operatorname{SDNN}\left(F_{5,156}=2.23 p=0.006\right)$ and $\operatorname{rMSSD}\left(F_{5,156}=2.53 p=\right.$ 0.031). However, these parameters were not significantly different between the TD and ASD "w/o med" groups or between "w/med" and "w/o med" ASD groups. Similar results between the "w/med" and "w/o med" groups were obtained in the NDD group.

\subsubsection{IQ Effect}

No significant IQ effect was observed on any PLR or HRV parameters in the ASD group. Children with ASD and a "Low IQ" had a slightly longer latency, lesser constriction amplitude, shorter constriction/redilation times, and smaller pupil diameter than those with a "High IQ" (Fig. 5-5). However, the MANOVA model indicated that the differences between the "High IQ" and "Low IQ" groups were only marginally significant with respect to PLR latency $\left(F_{4,98}=2.28 p=0.066\right)$ and not significant for constriction amplitude $\left(\mathrm{F}_{4,100}=0.37 p=0.83\right)$, constriction time $\left(\mathrm{F}_{4,97}=1.84 p=0.13\right)$, and redilation time $\left(\mathrm{F}_{4,95}=0.86 p=0.49\right)$. The TD group had significantly shorter latency, larger constriction amplitude, and longer constriction time than both the "High IQ" and "Low IQ" ASD groups.

Children with ASD and a "Low IQ" had a slower mean AHR, larger SDNN and rMSSD than those with a "High IQ" (Fig. 5-6). However, the MANOVA model indicated that the differences between the "High IQ" and "Low IQ" groups were insignificant $\left(\mathrm{F}_{5,88}\right.$ 
$=0.93 p=0.47$ for AHR; $F_{5,88}=0.51 p=0.77$ for SDNN; and $F_{5,88}=0.66 p=0.66$ for rMSSD). The TD group had significantly slower AHR than the "High IQ" ASD group $\left(F_{5,167}\right.$ $=4.21 p=0.001)$, but not the "Low IQ" ASD group $\left(F_{5,137}=1.72 p=0.13\right)$. An IQ effect was not found for any other PLR and HRV parameters in the ASD group. Similar results were observed in the NDD group.
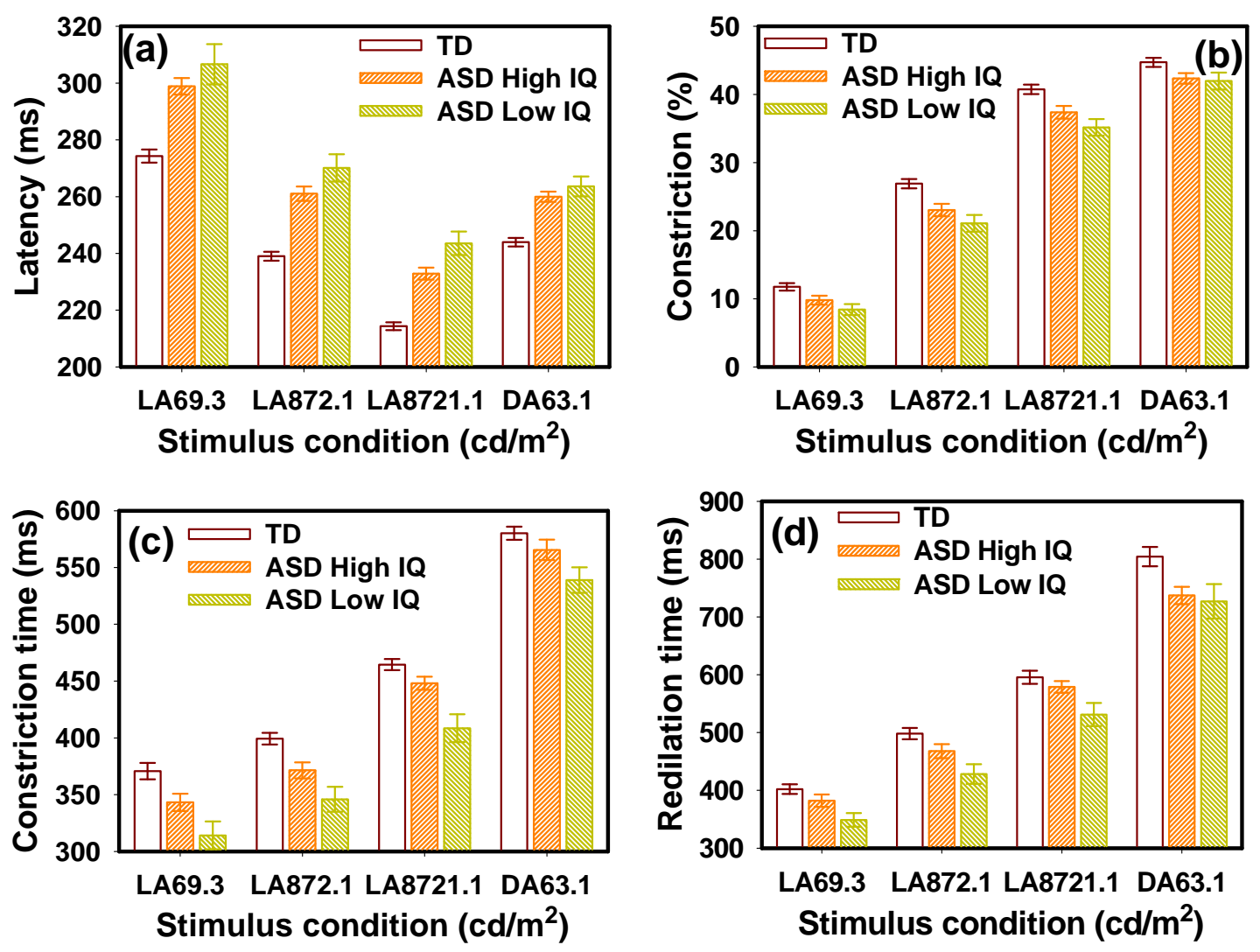

Fig. 5-5: The IQ effects on PLR parameters.

(a) PLR latency, (b) constriction amplitude, (c) constriction time and (d) redilation time in the ASD group. The error bars indicate the standard error. 

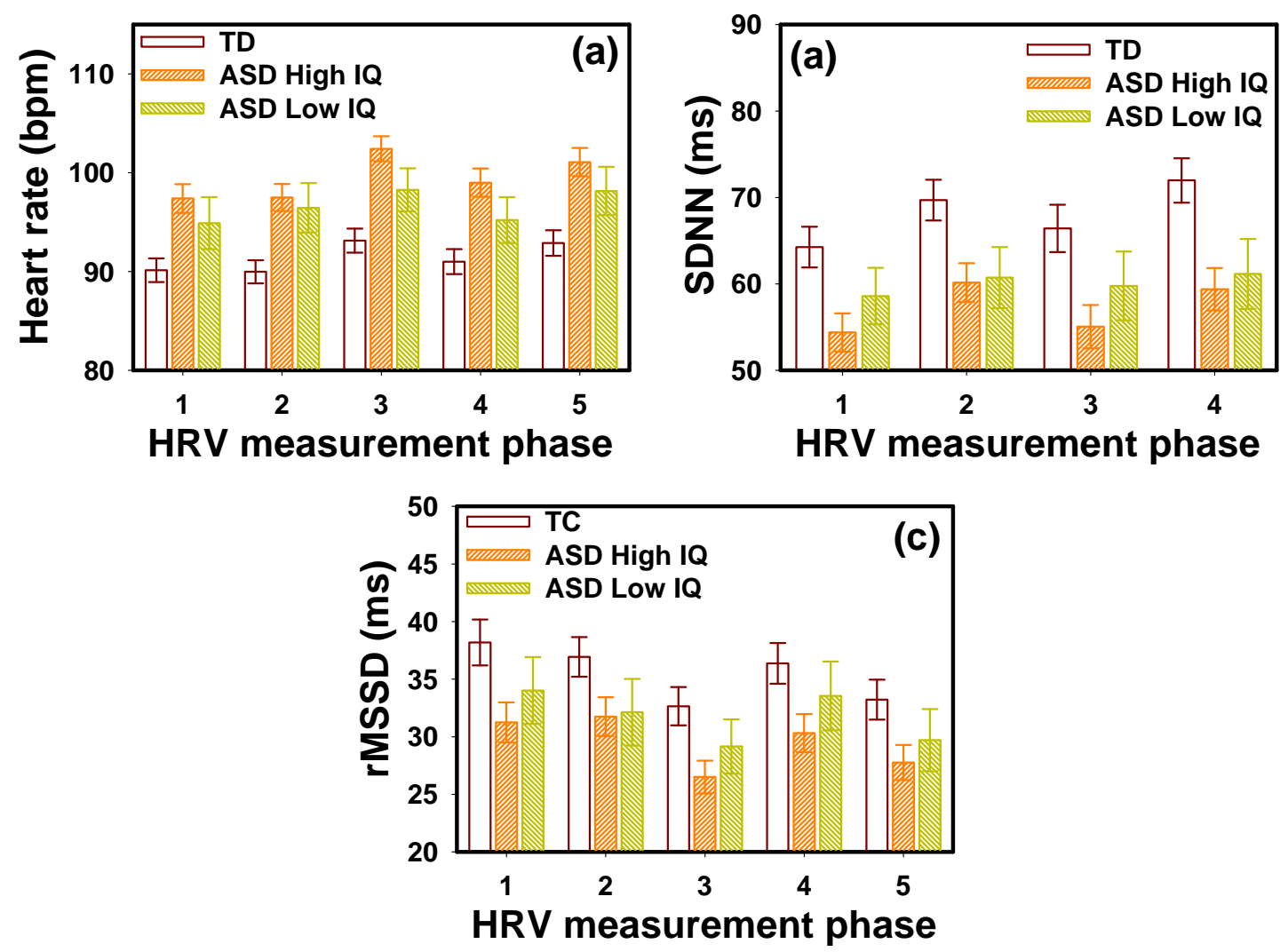

Fig. 5-6: The IQ effects on average heart rate and HRV parameters.

AHR, (b) SDNN and (c) rMSSD in the ASD group obtained in all five HRV measurement phases. The error bars indicate the standard error.

\subsubsection{Interaction between IQ and Medication}

The interaction between IQ and medication appeared to have a significant effect on PLR latency in the ASD group as revealed by ANOVA $\left(\mathrm{F}_{1,453}=12.74 p=0.0004\right)$ (Fig. 5-7). Children in the "High IQ" group did not show a difference with medication (MANOVA F $F_{4,87}=0.34 p=0.85$ ). In the "Low IQ" group, those using medication appeared to have a longer latency than those who were not using medication. However, this difference did not reach statistical significance in the MANOVA test $\left(F_{4,43}=1.40 p=\right.$ $0.25)$. 

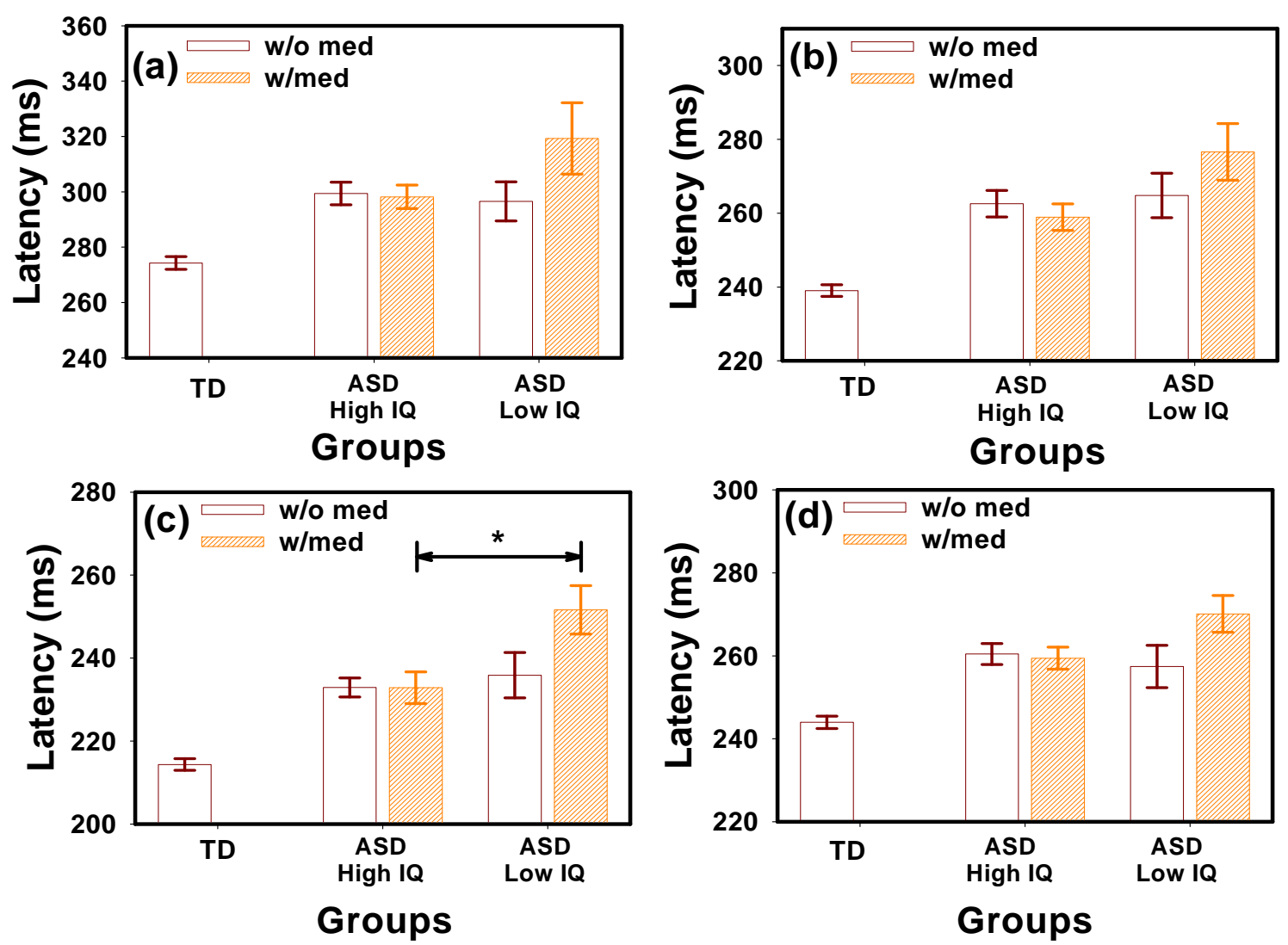

Fig. 5-7: The effect of IQ and medication interaction on PLR latency. At stimulation intensities of (a) $L A 69.3 \mathrm{~cd} / \mathrm{m}^{2}$, (b) $L A 872.1 \mathrm{~cd} / \mathrm{m}^{2}$ (c) $L A 8721.1 \mathrm{~cd} / \mathrm{m}^{2}$, and (d) DA63.1 cd/ $/ \mathrm{m}^{2}$ in the ASD group. ${ }^{*}$-test $p=0.03$, Bonferroni corrected.

Further analysis in the "w/o med" subgroups indicated that the "High IQ" group had a similar latency as the "Low IQ" group (MANOVA F $4,53=0.87 p=0.5$ ). However, the IQ effect was significant in the "w/med" group at the highest stimulus intensity of LA $8721.1 \mathrm{~cd} / \mathrm{m}^{2}$ (t-test $p=0.03$, Bonferroni corrected) with the "Low IQ" showing a longer latency than the "High IQ" group.

The above interaction effect was not significant on other PLR parameters or on any HRV parameters in the ASD group. In addition, the above interactions were not significant in the NDD group. 


\subsubsection{Effects of PLR Test on HRV}

The $\mathrm{HF}_{\mathrm{N}}$ and LF/HF parameters changed significantly when transiting between resting periods and PLR testing periods in all 3 groups. Such changes were smaller in the ASD and NDD groups than the TD group.

The ANCOVA model indicated that the HRV measurement phase had a statistically significant effect on AHR, SDNN, rMSSD, LF/HF, and normalized HF power $\left(\mathrm{F}_{4,1343}=6.29 p<0.0001 ; \mathrm{F}_{4,1340}=3.29 p=0.01 ; \mathrm{F}_{4,1340}=4.89 p=0.0006 ; \mathrm{F}_{4,1340}=8.84 p<\right.$ $0.0001 ;$ and $\mathrm{F}_{4,1320}=18.91 p<0.0001$, respectively). The interaction between group and HRV measurement phase was not significant. However, post-hoc one-way ANOVA indicated that the HRV measurement phase effect was significant only for the LF/HF $(p<$ $0.013)$ and $\mathrm{HF}_{\mathrm{N}}(p<0.005)$ in all 3 subject groups.

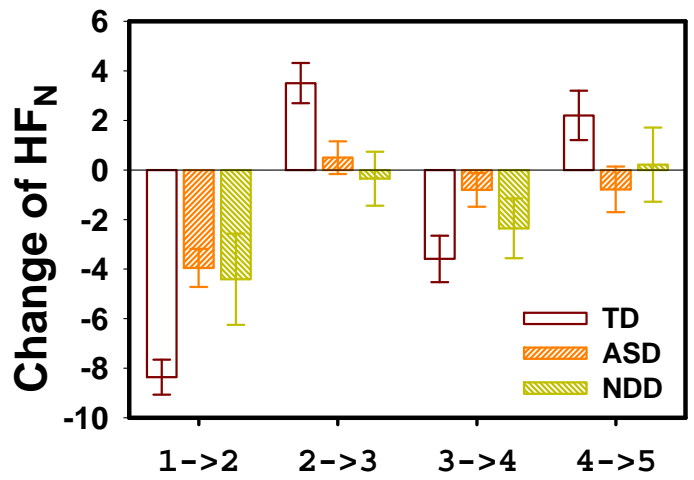

HRV measurement phases

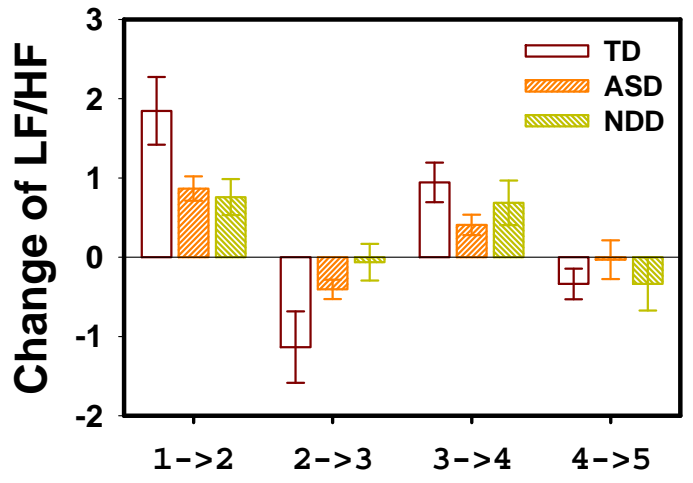

HRV measurement phases

Fig. 5-8: The change of frequency domain HRV parameters between consecutive HRV measurement phases.

(a) HF normalized power and (b) LF/HF ratio. The error bars indicate the standard error. The HRV measurement phases are numbered as 1: before PLR test, 2: during LA PLR, 3: during dark adaptation, 4: during DA PLR and 5: after PLR test. 
The changes of the 2 frequency domain parameters between 2 adjacent HRV measurement phases are shown in Fig. 5-8. $\mathrm{HF}_{\mathrm{N}}$ decreased when transiting from resting phases to test phases (phase 1 to 2 and phase 3 to 4 ) and increased when transiting from test phases to resting phases (phase 2 to 3 and phase 4 to 5). The changes in the $\mathrm{LF} / \mathrm{HF}$ parameters were opposite of those observed in $\mathrm{HF}_{\mathrm{N}}$. The $\mathrm{HF}_{\mathrm{N}}$ changes were significantly larger in the TD group than in the ASD groups (MANOVA $F_{4,226}=4.81 p=$ 0.001). However, the LF/HF ratio changes between the TD group and the ASD group was not significantly different (MANOVA $F_{4,231}=1.73 p=0.14$ ). The above changes were not significantly different between the ASD and NDD groups (MANOVA F $\mathrm{F}_{4,157}=0.81 p=0.52$; and $\mathrm{F}_{4,160}=0.99 p=0.42$ for $\mathrm{HF}_{\mathrm{N}}$ changes and LF/HF ratio changes, respectively).

\subsubsection{Correlation between PLR and HRV}

PLR constriction amplitude was significantly correlated with average heart rate in the ASD group in all LA tests $(r=-0.3, p<0.01)$ (Fig. 5-9). This correlation was observed in both the "w/o med" ASD and 'w/med" ASD groups. However, this correlation was not observed in typically developing children $(p>0.05)$. This correlation was significant in the NDD group only at the highest stimulus intensity of $L A 872.1 \mathrm{~cd} / \mathrm{m}^{2}$. Correlations were not found between other PLR and HRV parameters. 

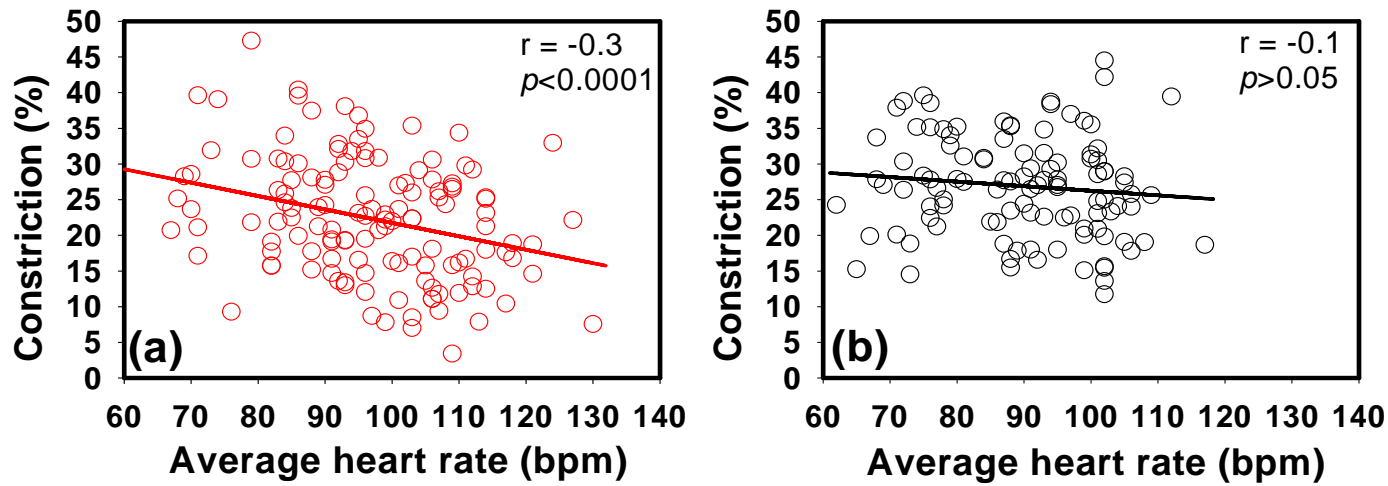

Fig. 5-9: The correlation between average heart rate and relative constricition amplitude.

In (a) children with ASD and (b) typical controls. The data shown were measured at stimulus intensity of LA $872.1 \mathrm{~cd} / \mathrm{m}^{2}$. (Pearson's $r=-0.3^{*},-0.3^{* *},-0.3^{* *},-0.1^{\mathrm{a}}$ in the ASD group and $r=-0.06^{a},-0.1^{a},-0.1^{a},-0.02^{a}$ in the TD group at stimulus LA $69.3 \mathrm{~cd} / \mathrm{m}^{2}$, LA $872.1 \mathrm{~cd} / \mathrm{m}^{2}$, LA $8721.1 \mathrm{~cd} / \mathrm{m}^{2}$, and DA $63.1 \mathrm{~cd} / \mathrm{m}^{2}$, respectively. ${ }^{*} p<0.001,{ }^{*} p<0.01,{ }^{a}$ $p>0.05)$.

\subsubsection{Subject Group Discrimination}

Using the DISCRIM procedure in SAS, a step-wise (PROC STEPDISC) variable selection procedure was used to identify the best candidate parameters to discriminate between the ASD and TD groups. With a significance level of $p=0.15$, the procedure selected following measurements for the discrimination model: latency at LA $69.3 \mathrm{~cd} / \mathrm{m}^{2}$ and LA $8721.1 \mathrm{~cd} / \mathrm{m}^{2}$, constriction amplitude at LA $69.3 \mathrm{~cd} / \mathrm{m}^{2}$ and LA $8721.1 \mathrm{~cd} / \mathrm{m}^{2}$, constriction time at LA $69.3 \mathrm{~cd} / \mathrm{m}^{2}$ and LA $872.1 \mathrm{~cd} / \mathrm{m}^{2}$, and resting pupil diameter at DA. The discriminant analysis results were significant $\left(\chi^{2}(28)=85.5, p<0.0001\right)$ with $81.5 \%$ subjects successfully classified ( $23.6 \%$ false negatives and $12.3 \%$ false positives). When the NDD group was included in the test data set, $72.4 \%$ of them were classified into the ASD group and $27.6 \%$ were classified into the TD group. Notably, the majority (53.8\%) of the misclassified children with typical development were female although females 
comprised only a small portion of the overall sample. A slightly higher successful discrimination rate $(83.4 \%)$ was obtained when the DISCRIM procedure was applied to the dataset after removing all female participants, with a $21.7 \%$ false-negative rate and a $9.0 \%$ false-positive rate. Examination of autism specific variables revealed that $9.3 \%$ children with classic autism were misclassified, along with $25 \%$ with Asperger's and 26.5\% with PDD-NOS. Of the children with ASD who were misclassified, $76.7 \%$ were in the "High IQ" group.

When logistic regression (PROC LOGISTIC) was performed in SAS using the same variables selected in step-wise variable selection procedure mentioned above, $79.8 \%$ subjects were successfully classified ( $22.3 \%$ false negatives and $17.6 \%$ false positives) and the area under the receiver-operating characteristic (ROC) curve was 0.88 which indicates good predictive ability (Fig. 5-10).

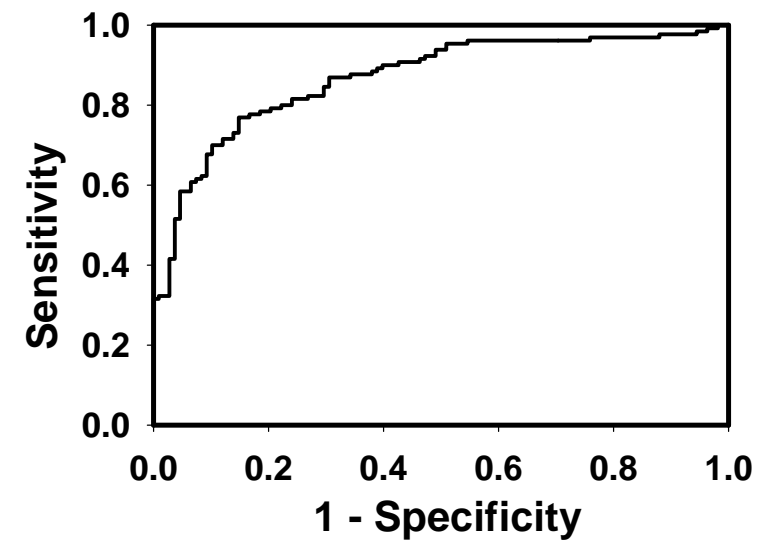

Fig. 5-10: The receiver-operating characteristic (ROC) curve for the logistic regression model. 


\subsection{Discussion}

The current results confirmed the previous observation by Fan et al. (2009a) that children with an ASD had longer latency and less relative constriction than children with typical development. Furthermore, we found that the constriction time and redilation time were shorter in children with ASD compared to children with typical development. Due to the predominance of male participants in this study, we also analyzed the data with only the male participants and all group differences remained the same. Our analyses did not show a significant difference between the PLR and HRV measurements obtained in the mornings and those obtained in the afternoons. We did not find any ASD diagnosis (classic autism, Asperger's Syndrome, and PDD-NOS) effects on PLR and HRV measurements.

It is interesting that the age trend of PLR latency observed in typically developing children was not observed in the ASD group. It is important to note that this trend (Fig. 5-1) is in sharp contrast to the age profiles of AHR and HRV (Fig. 5-2) which are similar in both TD and ASD groups. As an additional comparison, the age trend of PLR latency in typical controls was different from the maturation of the visual system characterized by pattern visual evoked potential (VEP), which stabilizes after 6 months of life (McCulloch and Skarf, 1991), but is similar to the trend observed in flash VEP (Dockstader et al., 2012). In addition, this age trend is coincident with the white matter maturation trend revealed in diffuse-tensor MRI studies (Bashat et al., 2007). It has been reported that children with an ASD have accelerated white matter maturation before 4 years of age (Bashat et al., 2007; Weinstein et al., 2011), but this trend is reversed after 4 years of 
age (Vissers et al., 2012). This appears to be consistent with our observation on PLR latency (Fig. 5-1).

We also observed a significant age effect on HRV parameters which has been previously widely reported (Massin and von Bernuth, 1997; Silvetti et al., 2001). The average heart rate is known to decrease with age, and time-domain HRVs (SDNN and rMSSD) were reported to increase with age (Silvetti et al., 2001). Massin and von Bernuth (1997) showed that HRV parameters changed rapidly during the first few years of life and eventually stabilized at older ages (6-15 years). Such age effects on HRV were generally attributed to the progressive maturation of the ANS (Silvetti et al., 2001). Our results suggested that the age effect on HRV was similar in the TD and ASD groups (Fig. 5-2), which is in sharp contrast to the different age profiles observed in PLR latency (Fig. $5-1)$

The ASD group showed a faster average heart rate than that of the typically developing controls, which is similar to previous findings (Palkovitz and Wiesenfeld, 1980; Kootz and Cohen, 1981; Ming et al., 2005; Bal et al., 2010). The faster average heart rate suggests an increased sympathetic tone and/or impaired parasympathetic control in children with an ASD. The study by Levy (1990) suggested that resting heart rate is predominantly controlled by vagal modulation. The ASD group also had smaller PLR constriction amplitude, indicating lower parasympathetic modulation (Barbur, 2004; Clarke, 2007). A previous cardiovascular study showed that children with an ASD had lower parasympathetic activity (Ming et al., 2005). Interestingly, a statistically significant negative correlation existed between PLR constriction and average heart rate in the ASD 
group but not in the typically developing children. This observed correlation may indicate possible parasympathetic dysregulation associated with ASD. Significant correlations between PLR and HRV parameters were also previously reported in adults with acute schizophrenia (Bär et al., 2008), but an unequivocal correlation was not found in healthy adults (Bär et al., 2009) or healthy children (Daluwatte et al., 2012a).

Frequency-domain HRV parameters appeared to change significantly when transiting between the rest and test phases in both the ASD and TD groups. Specifically, the $H F_{N}$ decreased during transition from a resting phase to a PLR test phase (1 to 2 and 3 to 4 ) and increased during transition from a testing phase to a resting phase ( 2 to 3 and 4 to 5). The LF/HF showed a reversed trend. This observation is similar to the previously reported posture-induced HRV changes associated with orthostatic stress (Mukai and Hayano, 1995; Montano et al., 1994; Yeragani et al., 1993). The PLR test requires the participant to incline slightly forward $\left(\sim 15^{\circ}\right)$, and this posture change can cause elevation in sympathetic tone due to muscle stress. Delaney and Brodie (2000) reported that psychological stress can increase low-frequency HRV while decreasing high-frequency HRV. Nevertheless, the observation of significantly smaller PLR testrelated HRV changes in the ASD group suggested less variability in vagal and sympathetic modulation in this population. This is similar to the results reported by Toichi and Kamio (2003) who found that typical controls showed a significant decrease in cardiac autonomic function during a mental arithmetic task while the ASD group did not show significant changes. The observation in the ASD group was not caused by medication because the conclusion remained the same with only the "w/o med" ASD 
group used in the data analysis.

The current results did not support a significant IQ effect on PLR parameters. The apparent IQ effect on PLR latency was complicated by the medication effects. The analysis of the interaction between the IQ and medication supported the notion that the IQ alone does not have a significant effect on PLR latency. In the "w/o med" ASD group, where the medication effect was excluded, those in the "High IQ" group showed similar latencies as those in the "Low IQ" group. Medication effect was not observed in the "High IQ" group; however, in the "Low IQ" ASD group, latency tended to be greater in children using medication than in those not using medication. Children in the "Low IQ" group may have required medications for their severe symptoms. In other words, the observed longer PLR latency in this group of participants ("Low IQ" and "w/med") may have been associated with their symptoms rather than with medication. A similar effect of IQ and medication interaction was not observed in other PLR and HRV parameters. A trend of medication effects was observed in the results especially on average heart rate and time-domain HRV parameters. However, the difference between "w/o med" and "w/med" ASD groups did not reach a statistical significance. Most of the children in the "w/med group" were taking multiple medications, which made it difficult to clarify the effect of individual medications. This observation requires further investigation.

The area under the ROC curve (greater than 0.8 ) suggests that the parameters used in the discrimination model have good predictive ability to predict whether the subject belongs to ASD group or not. However, the discrimination analysis results reported herein were not as robust as those reported by Fan et al. (2009a); this was 
most likely due to the increased sample size and the heterogeneity therein. The different age trends in the ASD and TD groups strongly suggested that age should be considered when interpreting PLR measurements. Despite the low number of participants in the non-ASD NDD group, our results indicated that the NDD group had similar PLR and HRV parameters as those of the ASD group. Therefore, the observed atypical PLR parameters were not specific to ASD. In other words, the same dysfunctions involved in the PLR pathway are most likely implicated in both ASD and other neurodevelopmental disorders.

\subsection{Conclusion}

We measured PLR and HRV simultaneously in a large heterogeneous group of children with an ASD, age-matched typically developing children, and children with an NDD other than an ASD. Children with an ASD or NDD showed atypical PLR, including greater latency, less constriction amplitude, and shorter constriction/redilation times. We also found a significant age effect in children with typical development that was not observed in children with an ASD; this may be due to altered brain development associated with ASD. Furthermore, we found a correlation between PLR and HRV parameters in the ASD group; this correlation was absent in children with typical development. These findings, in addition to atypical PLR profiles, suggest that an abnormality in the ANS is associated with ASD. The similar atypical PLR observed in ASD and NDD indicates that PLR differences are implicated in a wide range of neurodevelopmental disorders. As a simple and economic neurological test, PLR may be 
potentially useful for early screening of neurodevelopmental disorders in children. 


\section{CHAPTER 6}

\section{ASSOCIATION BETWEEN SENSORY PROCESSING AND PUPILLARY LIGHT REFLEX IN CHILDREN WITH AUTISM SPECTRUM DISORDERS}

\subsection{Introduction}

The pupil size is controlled by two antagonist iris muscles, the sphincter and dilator, which produce pupil constriction and dilation, respectively. Change in pupil size is largely under autonomic nervous system (ANS) control with the sphincter mainly innervated by the parasympathetic nervous system and the dilator innervated by the sympathetic system (Barbur, 2004). The sympathetic tract passes through the ciliary ganglion without synapsing and emerges as the long ciliary nerves entering the eye along the optic nerve. These postganglionic sympathetic nerves travel within the suprachoroidal space to innervate the iris dilator muscle (Appenzeller, 1999). The preganglionic parasympathetic nerve branches off to the ciliary ganglion and emerges as several short ciliary nerves which innervate the iris sphincter muscle (Appenzeller, 1999).

The dynamic changes in pupil size induced by optical luminance changes, referred to as pupillary light reflex (PLR), provides a simple yet reliable clinical assessment of ANS function (Barbur, 2004; Bremner, 2009). Recently atypical PLR has been described (Fan et al., 2009a; Daluwatte et al., 2012b) in children with autism 
spectrum disorders (ASDs). ASDs are complex developmental disorders with symptoms in three core areas: social functioning, communication, and restricted or repetitive behaviors. The finding of atypical PLR in ASD contributes to the increasing evidence of ANS dysfunction associated with ASD.

The ANS is involved in a multitude of physiological activities including sensory processing (Saper, 2002; Vallbo et al., 1979). The sensory system is an essential part of the neurological system that transduces the physical world to our perception. Human senses can be divided into five traditional groups (vision, auditory, taste, smell, and touch) and various non-traditional senses such as temperature and pain. Sensory dysfunction is common in children with ASD (Klintwall et al., 2011; Kientz and Dunn, 1997; Tomchek and Dunn, 2007). Some associations between sensory function and ASD symptoms have been investigated. For example, a correlation between degree of sensory dysfunction (total score on Short Sensory Profile) and amount of restricted and repetitive behaviors has been reported (Boyd et al., 2009; Chen et al., 2009).

Connections between sensory symptoms and some physiological measures have been previously explored. Jones et al. (2009) studied the association of sensory behaviors with auditory processing and found that poor performers on the intensity discrimination and those who performed well on duration discrimination had more difficulty coping with loudness levels. Fuentes et al. (2011) noted no correlation between sensory dysfunction and motor capability (elbow angle precision, fingertip precision). Woodard et al. (2012) reported an association between sensory processing and heart rate responses to a variety of sensory stimuli in both children with ASD and 
children with typical development.

Because the ANS is involved in regulating both sensory behavior and PLR, we investigated the potential association between PLR and sensory profile in children with ASD and children with typical development. We hypothesize that PLR parameters are associated with sensory behavior in children with ASD.

\subsection{Methods}

\subsubsection{Participants}

Sensory profile data was collected from a group of children who participated in a study where simultaneously measured PLR and heart rate data were obtained (Daluwatte et al., 2012b). Briefly, the study population included 152 children with ASD (referred to as the "ASD" group) and 107 children with typical development (referred to as the "TD" group). The age distribution of the ASD group is from 5 to 19 years with an average age of $10.7 \pm 3.4$ years, while the 107 TD children were from 6 to 17 years (10.9 \pm 2.9 years). There are 135 boys (10.9 \pm 3.5 years), 17 girls ( $9.8 \pm 2.6$ years) in the ASD group, and 79 boys ( $11.1 \pm 3.1$ years), 28 girls ( $10.6 \pm 2.4$ years) in the TD group. The ASD group was further divided between those who took some medication (stimulants, atypical antipsychotics, serotonin reuptake inhibitors, antihistamines, antiepileptics etc.) within 48 hours before the PLR test and those with no medication use. Seventy children were exposed to one or more medications and 82 were medication free. No one in the TD group had taken medication. ASD diagnoses included 86 with classic autism (autistic disorder), 32 Asperger syndrome and 34 PDD-NOS. 
This study was approved by the Institutional Review Board of the University of Missouri. All participants and their legal guardians provided written informed assent and consent prior to participating.

\subsubsection{Instrument and Procedure}

\subsubsection{PLR and Heart Rate Measurement}

The binocular pupillography recording system used to measure PLR was described previously in detail (Daluwatte et al., 2012b). The system used near-infrared cameras (GC660, Allied Vision Technologies, Stadtroda, Germany) to record pupil images at a speed of 115 frames-per-second (fps). PLR was stimulated using a 100 ms green light flash which was produced using $530 \mathrm{~nm}$ green LEDs. PLR was measured at four stimulation intensities in both light-adapted (LA) and dark-adapted (DA) conditions (LA $69.3 \mathrm{~cd} / \mathrm{m}^{2}$, LA $872.1 \mathrm{~cd} / \mathrm{m}^{2}$, LA $8721.1 \mathrm{~cd} / \mathrm{m}^{2}$ and DA $63.1 \mathrm{~cd} / \mathrm{m}^{2}$ ).

As explained by Daluwatte et al. (2012b), five PLR parameters (Fig. 1-2) were extracted: (1) resting pupil diameter; (2) PLR latency (time from stimulus to onset of constriction); (3) constriction time (time from onset of constriction to reach minimal diameter); (4) redilation time (time for the pupil to recover half of the constriction since minimal pupil diameter); (5) constriction amplitude (maximal relative change in pupil area during constriction). PLR parameters from both eyes during 8 repeated measurements were averaged to obtain the PLR parameters at each stimulus.

A wireless heart rate measuring device (Polar RS800CX, Polar Electro Oy, Finland) was used to obtain the heart rate. This device had a chest strap with an enclosed heart 
rate sensor that recorded the QRS intervals at a rate of $1 \mathrm{kHz}$. The average heart rate was calculated using data collected from $5 \mathrm{~min}$ before the PLR test until $5 \mathrm{~min}$ after the PLR test (total of 40 minutes).

\subsubsection{Sensory Processing}

A subset (29 items) sensory questionnaire was selected from the Sensory Profile (the "Caregiver Questionnaire" from Pearson Education, Inc., San Antonio, TX) (Dunn, 1999) to measure sensory profiles quantitatively in this study. The details of the sensory items used in this study are available from the National Database for Autism Research (http://ndar.nih.gov/ndarpublicweb/DataStructures.go?short_name=sensory_profile_1 99401). This questionnaire was completed by a parent or guardian for each participant. All items were rated on a Likert scale (1=Always, 2=Often, 3=Sometimes, 4=Rarely, and $5=$ Never). Sensory behavioral data were not available for 4 participants in the ASD group. The total sensory score was calculated by summing scores for each item and was used to represent overall sensory abnormality. A lower sensory total score indicates greater atypical sensory behavior.

\subsubsection{Statistical Analysis}

Reliability of the sensory total score was evaluated by calculating "Cronbach's Coefficient of Alpha" and an alpha value greater than 0.7 was considered reliable (Kline, 2000). The Kolmogorov-Smirnov test was used to verify normal distribution for all measured PLR and HRV parameters. The Wilcoxon Rank Sum test was used to compare 
the group differences in sensory total score between the two groups (ASD and TD). A p value $<0.05$ was considered significant.

To study the association between PLR parameters and sensory scores, linear correlations were first analyzed with the Spearman rank correlation (PROC CORR procedure in SAS). To investigate whether variations in PLR parameters can be explained by a combination of sensory behaviors, linear regression (PROC REG procedure in SAS) was applied with PLR parameters treated as dependent variables and sensory behaviors treated as independent variables.

The partial least squares (PLS) regression (the "PROC PLS" procedure in SAS) was performed to select a subset of sensory behavior (predictor variables) which explains the maximum variance in PLR parameters. Since the dataset consists of continuous response variables (PLR parameters) and ordinal predictor variables (sensory behaviors), the data processing method described by Russolillo and Lauro (2011) was used. A relatively small coefficient (absolute value $<0.04$ ) and a small variable importance for projection (VIP) statistic of Wold $(<0.8)$ were selected as the exclusion criteria for predictors. After selecting a subset of sensory items that best predict each PLR parameter, we calculated the sensory score A using the total score from these items and the sensory score B using the items which were not selected and reevaluated the Spearman rank correlation with the respective PLR parameters. Post-hoc one-way analysis of variance (ANOVA) was performed on PLR parameters while treating each selected subset of items as independent variable. Effect size (f) was calculated as 
explained by Cohen (1992) and 0.1, 0.25 and 0.4 were considered small, medium and large, respectively.

\subsection{Results}

The obtained Cronbach's Coefficient of Alpha was 0.9 for the ASD group and 0.8 for the TD group, suggesting the total sensory score was a reliable measure of sensory processing in both groups. The mean and standard deviations of the total sensory score in the ASD and TD groups were $98.5 \pm 16.5$ and $130.6 \pm 10.3$, respectively. The ASD group had a significantly lower total sensory score than that of the TD group $(p<0.05$ in Wilcoxon Rank Sum test), indicating greater atypical sensory behavior in children with ASD.

The Spearman rank correlation coefficients between total sensory score with each PLR parameter at each stimulus are summarized in Table 6-1. Fig. 6-1 provides a graphic rendition of the association between constriction amplitude and total sensory scores. PLR constriction amplitude was correlated with total sensory score in all lightadapted (LA) tests in the ASD group (Table 6-1 and Fig. 6-1a). However, this correlation was not observed in typically developing children (Table 6-1 and Fig. 6-1b). In the TD group, the light-adapted resting pupil size was negatively correlated with the total sensory score $(r=-0.22 p=0.0252)$ and the redilation time measured at DA 63.1 was positively correlated with the total sensory score $(r=0.19 p=0.0489)$. A statistically significant correlation between other PLR parameters and the sensory total score was not observed in either group (ASD and TD). 
Table 6-1: Spearman rank correlation coefficient between total sensory score and PLR parameters at each stimulus in TD and ASD groups.

\begin{tabular}{|c|c|c|c|c|c|}
\hline \multirow{7}{*}{ 号 } & & \multicolumn{4}{|c|}{ Stimulus $\left(\mathrm{cd} / \mathrm{m}^{2}\right)$} \\
\hline & & LA 69.3 & LA 872.1 & LA 8721.1 & DA 63.1 \\
\hline & Resting pupil diameter & & $-0.09^{\mathrm{a}}$ & & $0.008^{\mathrm{a}}$ \\
\hline & Latency & $-0.12^{a}$ & $-0.16^{a}$ & $-0.08^{a}$ & $-0.02^{\mathrm{a}}$ \\
\hline & Constriction time & $-0.04^{a}$ & $0.10^{\mathrm{a}}$ & $0.003^{a}$ & $0.05^{\mathrm{a}}$ \\
\hline & Redilation time & $0.07^{a}$ & $0.12^{a}$ & $-0.005^{a}$ & $0.06^{\mathrm{a}}$ \\
\hline & Constriction amplitude & $0.20 *$ & $0.25 * *$ & $0.26 * *$ & $0.07^{\mathrm{a}}$ \\
\hline \multirow{5}{*}{ 은 } & Resting pupil diameter & & $-0.22 *$ & & $-0.13^{a}$ \\
\hline & Latency & $0.05^{a}$ & $-0.08^{a}$ & $-0.03^{a}$ & $-0.14^{\mathrm{a}}$ \\
\hline & Constriction time & $-0.004^{a}$ & $-0.02^{a}$ & $-0.05^{a}$ & $0.11^{\mathrm{a}}$ \\
\hline & Redilation time & $-0.03^{a}$ & $-0.04^{a}$ & $0.14^{a}$ & $0.19 *$ \\
\hline & Constriction amplitude & $0.02^{a}$ & $-0.06^{a}$ & $0.003^{a}$ & $0.05^{\mathrm{a}}$ \\
\hline
\end{tabular}

LA = light adapted stimulus condition, DA = dark adapted stimulus condidtion

$* * p<0.01, * p<0.05,{ }^{a} p>0.05$
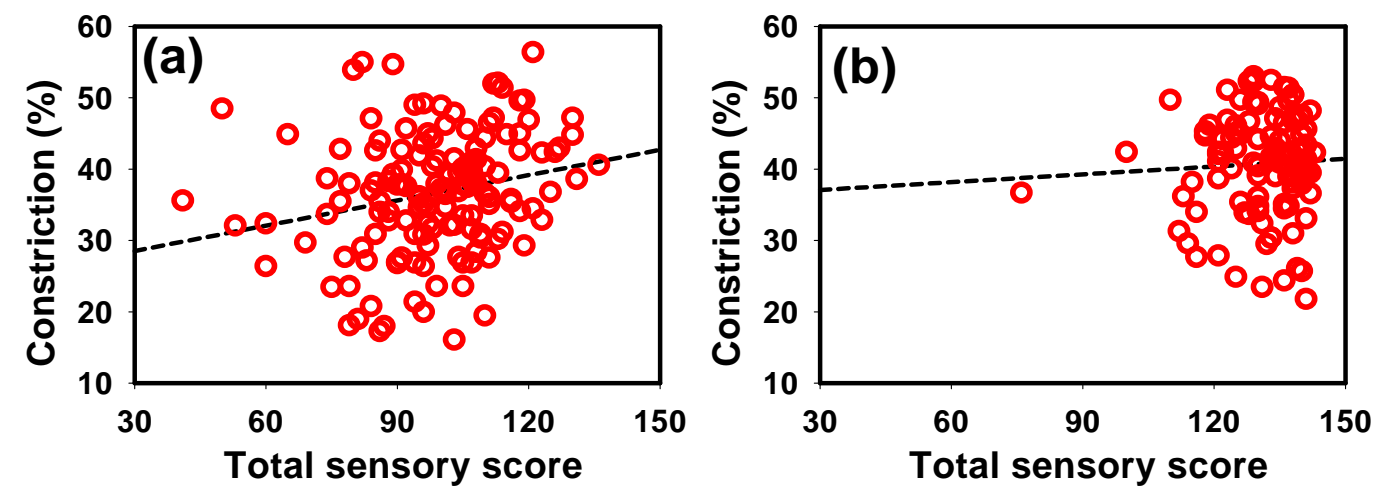

Fig. 6-1: The correlation between PLR constriction amplitude and total sensory score. In the (a) ASD and (b) TD groups. The Spearman rank correlation $r=0.26, p<0.01$ in the ASD group; $r=0.003, p>0.05^{a}$ in the TD group at LA $8721.1 \mathrm{~cd} / \mathrm{m}^{2}$. Lower sensory scores indicate greater atypical sensory behavior.

Since the most consistent correlation was found between constriction amplitude and the total sensory score in children with ASD, we further studied the correlation in the subgroups of this population (Table 6-2). This correlation was statistically significant for children with ASD who were not exposed to medications before testing, but not for the medication group. In addition, this correlation was consistently significant for the Autism group, but not in Asperger's or PDD-NOS groups (Table 6-2). None of the other 
PLR parameters demonstrated significant correlation with sensory scores, when divided by medication use or ASD diagnosis.

Table 6-2: Spearman rank correlation coefficient between total sensory score and PLR constriction amplitude at each stimulus in ASD sub-groups.

\begin{tabular}{lcccc}
\hline & \multicolumn{4}{c}{ Stimulus $\left(\mathrm{cd} / \mathrm{m}^{2}\right)$} \\
Medication & LA 69.3 & LA 872.1 & LA 8721.1 & DA 63.1 \\
\cline { 2 - 5 } With medication & & & & \\
Without medication & $0.22^{\mathrm{a}}$ & $\mathbf{0 . 3 0 ^ { * * }}$ & $\mathbf{0 . 3 3 ^ { * * }}$ & $0.14^{\mathrm{a}}$ \\
Diagnosis & $0.13^{\mathrm{a}}$ & $0.19^{\mathrm{a}}$ & $0.18^{\mathrm{a}}$ & $0.01^{\mathrm{a}}$ \\
$\quad$ & & & & \\
Autism & & & $0.36^{* *}$ & $0.09^{\mathrm{a}}$ \\
Asperger & $\mathbf{0 . 2 3 ^ { * }}$ & $\mathbf{0 . 2 7 ^ { * }}$ & $0.16^{\mathrm{a}}$ & $0.14^{\mathrm{a}}$ \\
PDD-NOS & $0.11^{\mathrm{a}}$ & $0.02^{\mathrm{a}}$ & $0.09^{\mathrm{a}}$ & $0.00^{\mathrm{a}}$ \\
\hline
\end{tabular}

LA = light adapted stimulus condition , DA = dark adapted stimulus condidtion ${ }^{*} p<0.01,{ }^{*} p<0.05,{ }^{\mathrm{a}} p>0.05$

PLR constriction amplitude measured at stimulus LA $8721.1 \mathrm{~cd} / \mathrm{m}^{2}$ showed the most significant correlation (highest $r$ value and smallest $p$ value) with the total sensory score. Regression analysis was used to determine the sensory measures that best predicted constriction amplitude at this stimulus condition in the ASD group. Two items, "avoids getting messy" ( $b=1.4 p=0.017)$ and "has difficulty paying attention" $(b=1.8 p$ $=0.005)$ from the Sensory Profile were the best predictors. The combination of these variables resulted in an $\mathrm{R}^{2}=0.09$. The residuals from the regression analysis were normally distributed.

Post-hoc one-way ANOVA confirmed significant effects from the items "Avoids getting messy" and "Difficulty paying attention", respectively on constriction amplitude at stimulus LA $8721.1 \mathrm{~cd} / \mathrm{m}^{2}$ in the ASD group $\left(\mathrm{F}_{1,142}=4.93 p=0.028 f=0.03\right.$ and $\mathrm{F}_{1,142}=7.05$ $p=0.0088 f=0.05$ for items "Avoids getting messy" and "Has difficulty paying attention", 
respectively). As shown in Fig. 6-2a and Fig. 6-2b, the constriction amplitude increased with the sensory scores on "Avoids getting messy" and "Has difficulty paying attention". At scores 4 and 5 (rarely and never), the constriction amplitude obtained in the ASD group was closer to that obtained in the TD group.
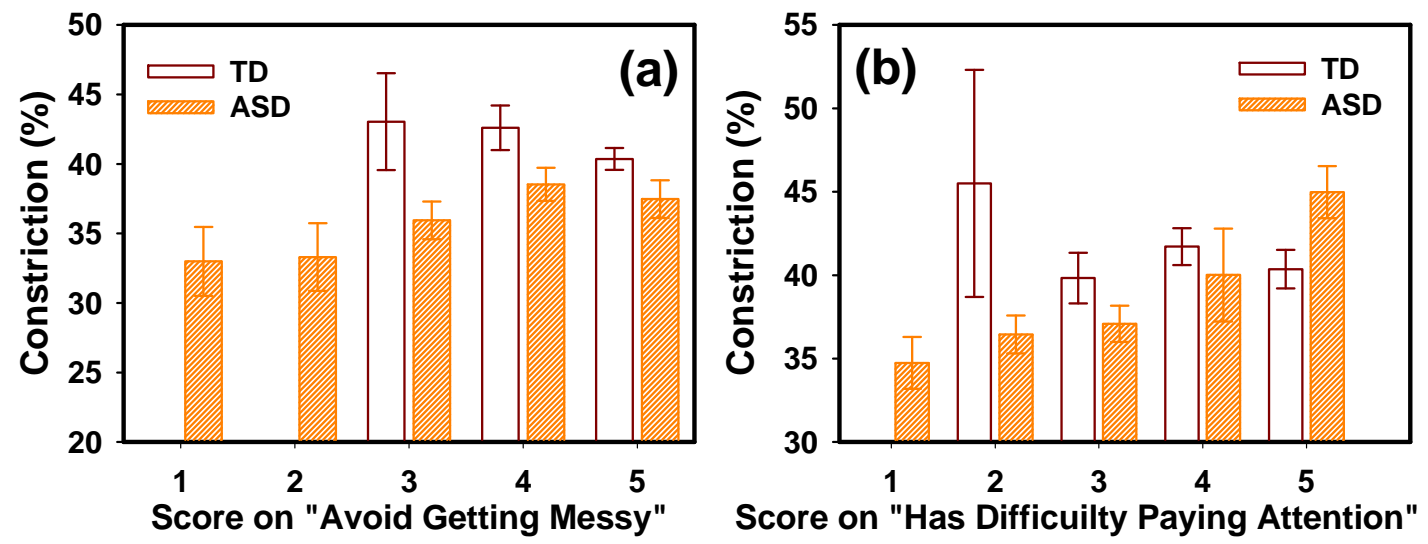

Fig. 6-2: PLR constriction amplitude at different scores for behavior items. (a) Avoid getting messy and (b) Has difficulty paying attention. The error bars indicate the standard error. Score key: $1=$ =Always, $2=$ Often, $3=$ Sometimes, $4=$ Rarely and $5=$ Never.

PLS regression was performed in the ASD group to select the subset of sensory behaviors which explains the maximum variance in constriction amplitude at stimulus LA $8721.1 \mathrm{~cd} / \mathrm{m}^{2}$. The following sensory behaviors were selected from the PLS regression model: "Makes repetitive noises", "Holds hands over ears to protect ears from sound", "Expresses discomfort with or avoids bright lights", "Looks carefully or intensely at objects/people", "Becomes anxious or distressed when feet leave the ground", "Avoids getting messy", "Expresses distress during grooming", "On the go", and "Has difficulty paying attention". This subset explained $11.1 \%$ of the variance in constriction amplitude. The PLS regression coefficients are reported in Table 6-3. The 
importance for projection (VIP) statistic of Wold is higher than 0.8 for each listed sensory item (predictor variable).

Table 6-3: Parameter estimates obtained from the PLS regression model and Post-hoc ANOVA analysis for constriction amplitude in the ASD group.

\begin{tabular}{|c|c|c|c|c|}
\hline \multirow[b]{2}{*}{ Sensory Behavior } & \multirow{2}{*}{$\begin{array}{c}\text { PLS } \\
\text { regression } \\
\text { coefficient }\end{array}$} & \multicolumn{3}{|c|}{ Post-hoc ANOVA } \\
\hline & & $\mathbf{F}$ & $p$ & $\begin{array}{c}\text { Effect size } \\
(f)\end{array}$ \\
\hline Makes repetitive noises & 0.05 & 4.09 & 0.045 & 0.03 \\
\hline Holds hands over ears to protect ears from sound & 0.05 & 3.56 & 0.061 & 0.02 \\
\hline Expresses discomfort with or avoids bright lights & 0.05 & 3.73 & 0.056 & 0.03 \\
\hline Looks carefully or intensely at objects/people & 0.05 & 3.65 & 0.058 & 0.03 \\
\hline Becomes anxious or distressed when feet leave the ground & 0.05 & 2.97 & 0.087 & 0.02 \\
\hline Avoids getting "messy" & 0.06 & 4.93 & 0.028 & 0.03 \\
\hline Expresses distress during grooming & 0.07 & 4.50 & 0.036 & 0.03 \\
\hline "On the go" & 0.08 & 6.69 & 0.011 & 0.04 \\
\hline Has difficulty paying attention & 0.07 & 7.05 & 0.009 & 0.05 \\
\hline
\end{tabular}

Post-hoc one-way ANOVA confirmed significant effects for 4 items out of the aforementioned 9 sensory items in Table 6-3: "Avoids getting messy", "Expresses distress during grooming", "On the go", and "Has difficulty paying attention". Children with ASD who reported "rarely" or "never" on the aforementioned four sensory items had a higher PLR constriction than those who reported "always". Even though with only marginally statistical significance, such trends were also observed with the other five sensory items.

The correlation between constriction amplitude and sensory behaviors was reevaluated by comparing two different sensory score groups. Group A was calculated using scores from the subset of sensory items selected by the PLS regression model (Table 6-3); whereas score B was calculated using scores from sensory items not selected by the PLS regression model. The PLR constriction amplitude was correlated 
with both score A and score B in all LA tests (Table 6-4). The correlation with Sensory score A showed a higher significance than Sensory score B.

Table 6-4: Spearman rank correlation $r$ for the correlation of PLR constriction amplitude with sensory score A and sensory score B in ASD group.

\begin{tabular}{lcccc}
\hline & \multicolumn{4}{c}{ Stimulus $\left(\mathrm{cd} / \mathrm{m}^{2}\right)$} \\
LA 69.3 & LA 872.1 & LA 8721.1 & DA 63.1 \\
\cline { 2 - 5 } Sensory score A & $\mathbf{0 . 1 7 ^ { * }}$ & $\mathbf{0 . 3 0 ^ { * * }}$ & $\mathbf{0 . 3 3 ^ { * * }}$ & $0.08^{\mathrm{a}}$ \\
Sensory score B & $0.20^{*}$ & $0.21^{*}$ & $0.20^{*}$ & $0.06^{\mathrm{a}}$ \\
\hline $\mathrm{LA}=$ light adapted stimulus condition $\left(\mathrm{cd} / \mathrm{m}^{2}\right), \mathrm{DA}=$ & dark adapted stimulus condidtion \\
$\left(\mathrm{cd} / \mathrm{m}^{2}\right)$ & & & & \\
${ }^{*} p<0.05,{ }^{*} * p<0.001,{ }^{a} p>0.05$ & & &
\end{tabular}

The average heart rate also correlated with the total sensory score $(r=-0.3, p<$ 0.05) (Fig. 6-3a) in the ASD group. However, this correlation was not observed in typically developing children ( $p>0.05)$ (Fig. 6-3b).
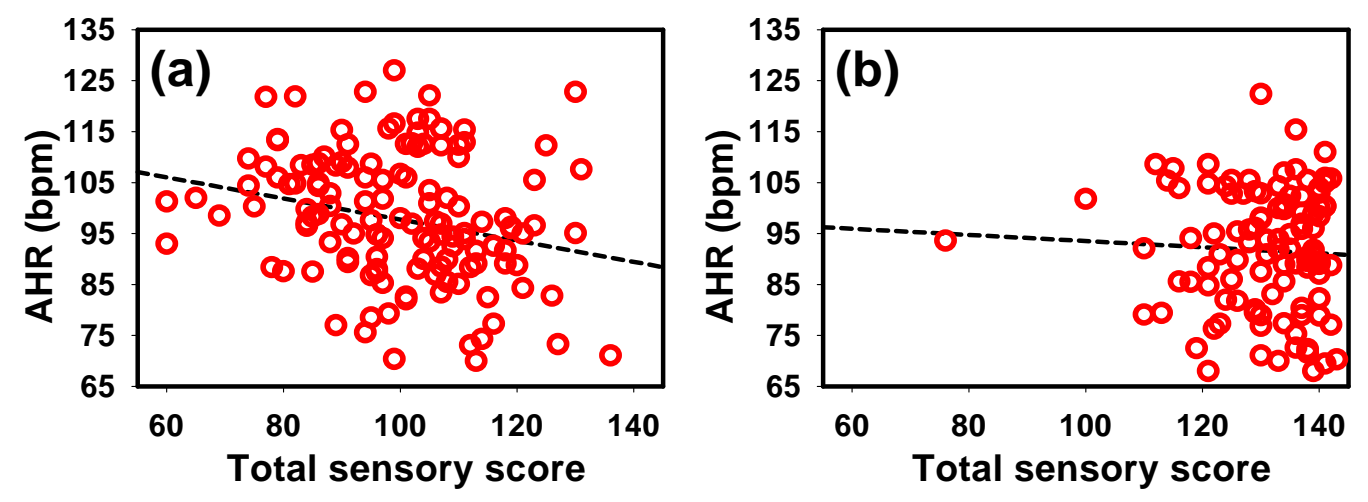

Fig. 6-3: The correlation between average heart rate and sensory total score. In the (a) ASD and (b) TD groups. Spearman rank correlation $r=-0.3, p<0.01$ in the ASD group; $r=-0.03 p>0.05$ in the TD group.

\subsection{Discussion}

Our results substantiate that children with ASD commonly have significant sensory dysfunction compared with children of typical development and indicate that 
sensory dysfunction may be caused or exacerbated by autonomic nervous system dysfunction. We found that ASD children with more atypical sensory behaviors (i.e. lower sensory scores) also had smaller PLR constriction amplitudes. Previously, we reported that children with ASD generally have lower smaller constriction amplitudes (Fan et al., 2009a; Daluwatte et al., 2012b) suggesting a lower parasympathetic modulation since PLR constriction is predominantly under parasympathetic control (Barbur, 2004; Clarke, 2007). Interestingly, the correlation between PLR constriction and the sensory score only occurred for children with ASD who were not exposed to medication prior to testing, and not in ASD children exposed to medications or in typically developing children. The disappearance of the correlation in medication-taking children is likely attributed to medication effects on the ANS. We were unable to tease out these effects since most children on medication were on multiple medications.

Among the four items that were selected in PLS regression as the best predictors of a smaller constriction amplitude, both "On the go" and "Has difficulty paying attention" are also considered as symptoms of attention-deficit/hyperactivity disorder (ADHD) (Dalsgaard, 2012). In post-hoc analysis we saw that children with ASD who reported "rarely" or "never" on these two items had a higher PLR constriction amplitude than those who reported "always", which seems to link such abnormal sensory behavior with impairment in parasympathetic function. Coincidentally, an abnormal parasympathetic mechanism has been reported previously in children with ADHD (Musser et al., 2011; van Lang et al., 2007). Musser et al. (2011) studied emotion regulation in ADHD using respiratory sinus arrhythmia ("RSA", a measure of 
parasympathetic reactivity) and cardiac pre-ejection period (a measure of sympathetic reactivity). Both ASD and TC groups had similar sympathetic reactivity. However, in response to positive emotion, typically developing children showed a decrease in RSA; whereas children with ADHD had an increase in RSA. van Lang et al. (2007) also reported that ADHD children had lower heart rate reactivity in response to emotional stress; while no significant difference was observed in skin conductance, a sympathetic measure. Since ADHD symptoms exist frequently in children with ASD (Murray, 2010), it is likely that such sensory problems in children with ASD may be also attributed to parasympathetic dysfunction.

ANS dysfunction in ASD was initially observed in cardiovascular data (Ming et al., 2005). Several studies reported a higher average heart rate in children with ASD than in the typically developing controls (Daluwatte et al., 2012b; Palkovitz and Wiesenfeld, 1980; Kootz and Cohen, 1981; Ming et al., 2005; Bal et al., 2010). A higher heart rate suggests increased sympathetic tone or/and diminished parasympathetic control. The current finding of a negative correlation between the heart rate and sensory total score in ASD but not in the typically developing children is consistent. Woodard et al. (2012) also reported a strong negative correlation between the Infant/Toddler Sensory Profile (Dunn, 2002), sensory processing domain and AHR in children with ASD, but no correlation in typically developing children. The lack of correlation in children of typical development suggests that the various components of the autonomic nervous system (PLR and cardiovascular) function relatively independently in healthy children. Whereas, in ASD some generalized impairment of parasympathetic modulation may override 
normal control thus contributing to sensory dysfunction. It is also possible the current testing techniques are not precise enough to identify similar associations in our control group of typical children. Finally, we have reported that heart rate and PLR constriction amplitude are negatively correlated in ASD (Daluwatte et al., 2012b). This was confirmed in this sample (data not shown) which is expected and important since both assess ANS function, specifically the balance of parasympathetic and sympathetic control.

We questioned why constriction amplitude in light adaption was the only PLR parameter which showed consistent association with the sensory profile. Both constriction time and constriction amplitude are indicators of parasympathetic modulation (Barbur, 2004) and are correlated (Pearson product moment correlation: $r \approx-$ 0.5, $p<0.0001$ for both ASD and TD groups). However, no association between constriction time and the sensory score was observed. This may suggest that constriction time and amplitude assess different information. Constriction amplitude measures the amount of response, while constriction time mainly indicates the speed of the response. Since the sensory assessment may also be considered an indicator of the degree of sensory responses, these results may be functionally consistent. In addition, considering the statistical variance of each PLR parameter, we note the constriction amplitude has the highest coefficient of variance (0.23-0.64 in ASD group). This may suggest constriction amplitude reflects the ANS fluctuations better than other PLR parameters. It is interesting to note that no significant correlation with the constriction amplitude was observed in the dark adaptation condition. This is most likely due to the 
saturation effect in the PLR response obtained in the dark. Consistent with this supposition, the coefficient of variance of constriction with dark adaptation is smaller than in light adaptation (0.18 vs $0.23-0.64)$.

It is interesting to note that the correlation between sensory behavior and PLR constriction was significant in the autism group, but not in Asperger's group or PDD-NOS group. This observation requires further investigation because of the relatively small number of subjects in the Asperger group and PDD-NOS group.

\subsection{Conclusion}

In summary, we studied associations between PLR and heart rate with sensory dysfunction in children with ASD and typical development. For children with ASD the degree of sensory dysfunction significantly correlated with decreased PLR constriction amplitude and increased resting heart rate. Such correlations were not observed in children with typical development. These results suggest that abnormal sensory behavior is associated with ANS dysfunction in ASD. One limitation of this study was the limited number of sensory questions used. Performing the PLS regression with a complete Sensory Profile (Dunn, 1999) is expected to better elucidate the association of sensory behaviors with the measure of ANS dysfunction. 


\section{CHAPTER 7}

\section{SUMMARY AND FUTURE DIRECTIONS}

In this dissertation research we improved the PLR system to automatically and more efficiently process pupil images (complete and incomplete). We made the software interface user-friendly so that its use does not need extensive training. A series of systematic studies were then carried out to investigate PLR in children with ASD using this improved system.

We measured simultaneously PLR and HRV in 152 children with ASD, 107 with typical development and 36 children with non-ASD neurodevelopmental disorders. The results confirmed previously reported atypical PLR in ASD children and also revealed atypical PLR in children with other neurodevelopmental disorders.

We found PLR latency decreased consistently with age in typically developing children from 6 to 9 years old. Interestingly this trend was coincident with the white matter maturation trend observed in a brain imaging study (Bashat et al., 2005). In children with ASD no such age dependent trend was observed, which suggests possible abnormal white matter developmental trajectory in ASD (Bashat et al., 2007). A study that simultaneously measures PLR and brain imaging in the same population can be helpful for further understanding whether the age trend observed in PLR latency is associated with white matter maturation. There are reports of atypical synaptic transmission in ASD (Zoghbi and Bear, 2012), which might also cause a delay in signal 
conduction, and thus result in prolonged PLR latencies. Future studies investigating the association between synaptic transmission and PLR can provide further insights on this issue.

While our findings showed the potential of using PLR as a simple noninvasive functional test to monitor neurodevelopment in children, PLR in children younger than six years needs to be studied to investigate how PLR latency changes with age in infants and toddlers. A longitudinal study of PLR is ideal to test whether PLR can monitor neurodevelopmental trajectory. In addition, it will be interesting to test whether PLR parameters are altered by treatment to ASD. Such studies can provide a better understanding of the potential of PLR as a treatment indicator.

By testing PLR and HRV simultaneously in a group of age and gender matched typically developing children, we found that variations of PLR and HRV in healthy children are not associated. This suggests that in healthy children these two measurements provide complementary information about the autonomic nervous system. We also observed that PLR constriction amplitude and average heart rate are correlated in children with ASD. In addition, PLR constriction amplitude is correlated with the sensory total score (behavioral observation) in the ASD group but not in children with typical development. These results suggest a profound impact of ANS dysfunction in multiple systems (PLR, cardiovascular system, and sensory processing) in children with ASD. While this was the first study which revealed association of PLR with behavior, further investigations focusing on other behavioral and clinical measures will lead to a better understanding of such associations in children with ASD. 
In children with ASD, we did not observe a significant medication effect or IQ effect in PLR. But a medication and IQ interaction effect was observed. There are previous reports of several medication effects on PLR. For example, Bitsios et al., (1999) showed that antidepressants prolonged PLR latency, decreased constriction amplitude and shortened the $75 \%$ recovery time of the PLR with venlafaxine. Theofilopoulos et al., (1995) showed similar results with desipramine and reboxetine. A majority of children on medication in our test population were on multiple medications, making it impossible to isolate the effect of each individual medication. Thus, the medication effect on PLR needs further systematic investigations. Finally, synaptic transmission abnormality has been linked to intellectual disability in ASD (Zoghbi and Bear, 2012). Therefore it is worthwhile to further investigate whether prolonged PLR latency observed in both groups (ASD and non-ASD neurodevelopment disorders) is related to intellectual development. 


\title{
APPENDIX A COPYRIGHT
}

\section{A.1 Journal of Autism and Developmental Disorders}

\author{
SPRINGER LICENSE \\ TERMS AND CONDIIIONS
}

Jan 23, 2013

This is a License Agreement between Chathuri L Dahwwatte ("You") and Springer ("Springer") provided by Copyright Clearance Center ("CCC"). The license consists of your order details, the terms and conditions provided by Springer, and the payment terms and conditions.

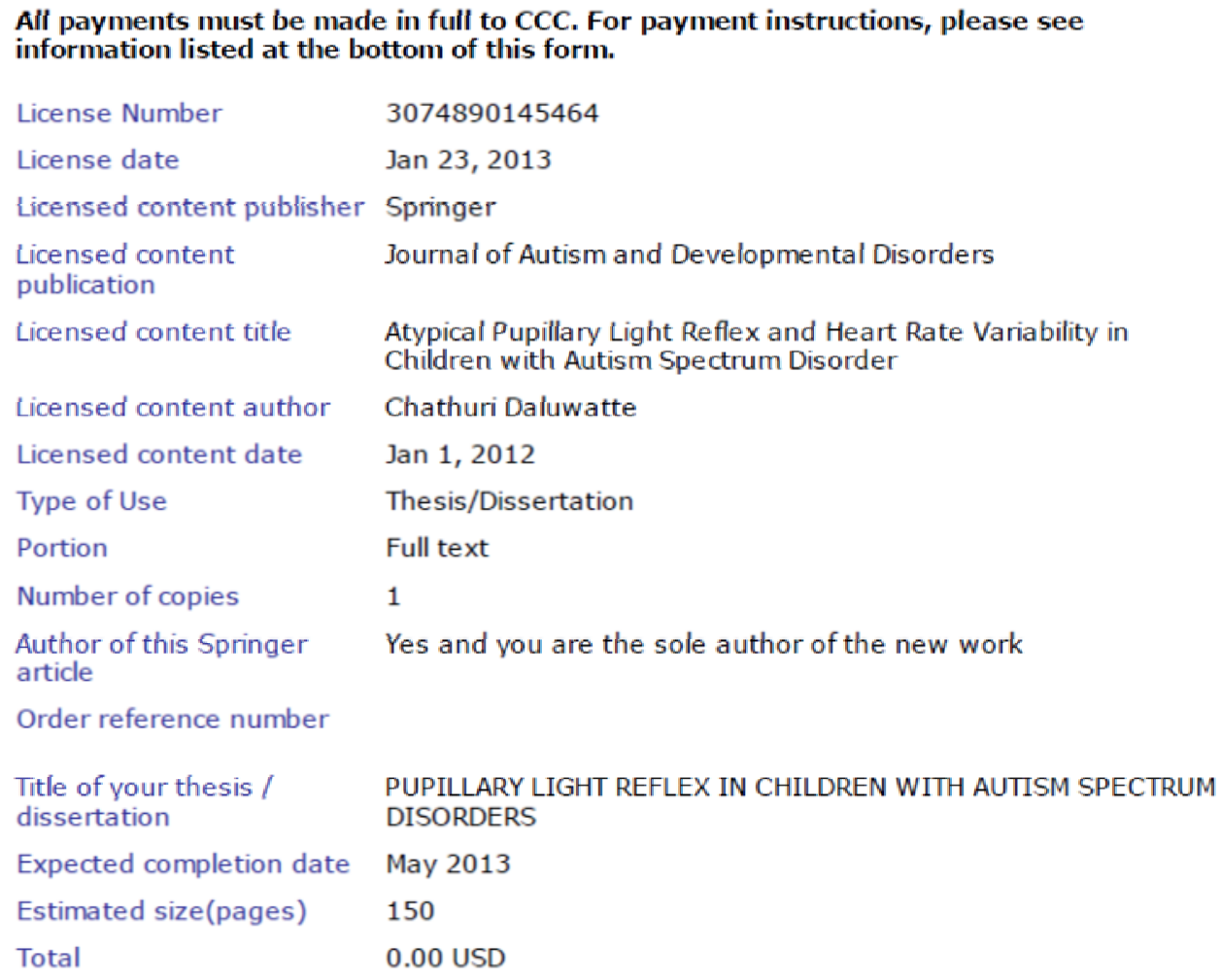

Total

0.00 USD

Terms and Conditions 


\section{A.2 IEEE Xplore}

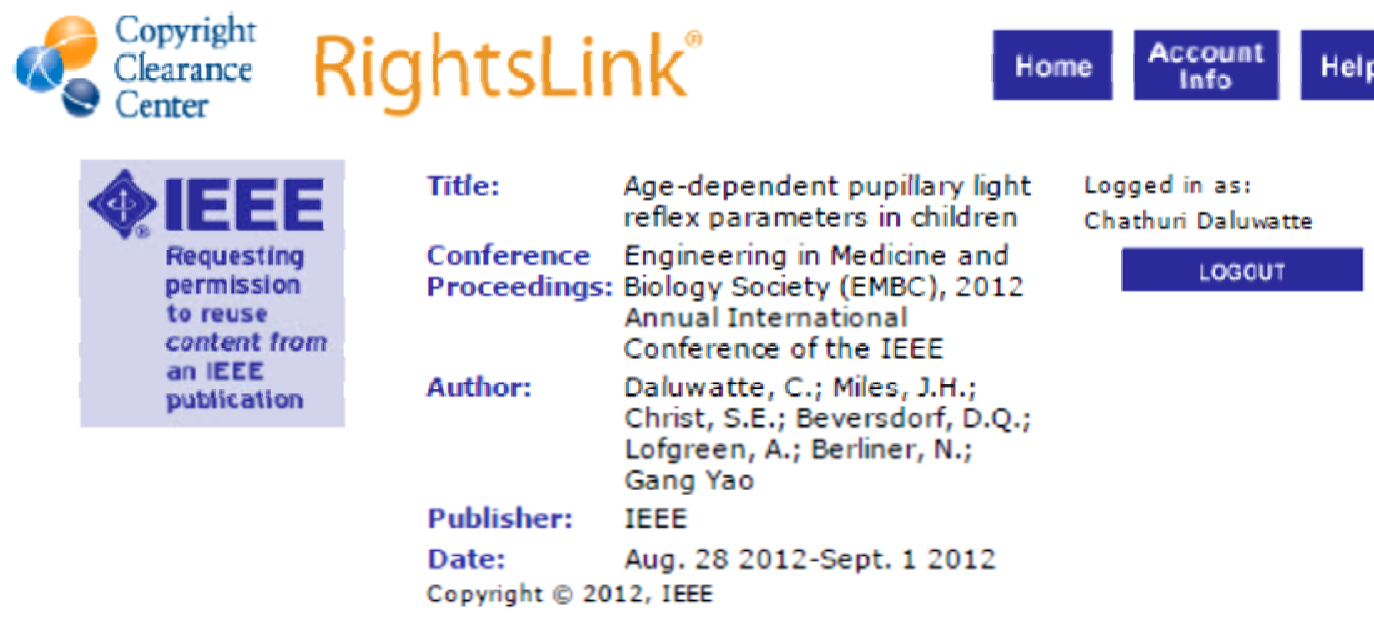

Thesis / Dissertation Reuse

The IEEE does not require individuals working on a thesis to obtain a formal reuse license, however, you may print out this statement to be used as a permission grant:

Requirements to be followed when using any portion (e.g., figure, graph, table, or textual material) of an IEEE copyrighted paper in a thesis:

1) In the case of textual material (e.g., using short quotes or referring to the work within these papers) users must give full credit to the original source (author, paper, publication) followed by the IEEE copyright line (c) 2011 IEEE.

2) In the case of illustrations or tabular material, we require that the copyright line (c) [Year of original publication] IEEE appear prominently with each reprinted figure and/or table.

3 ) If a substantial portion of the original paper is to be used, and if you are not the senior author, also obtain the senior author's approval.

Requirements to be followed when using an entire IEEE copyrighted paper in a thes is:

1) The following IEEE copyright/ credit notice should be placed prominently in the references: (C) [year of original publication] IEEE. Reprinted, with permission, from [author names, paper title, IEEE publication title, and month/year of publication]

2) Only the accepted version of an IEEE copyrighted paper can be used when posting the paper or your thesis on-line.

3) In placing the thesis on the author's university website, please display the following message in a prominent place on the website: In reference to IEEE copyrighted material which is used with permission in this thesis, the IEEE does not endorse any of [university/educational entity's name goes here]'s products or services. Internal or personal use of this material is permitted. If interested in reprinting/republishing IEEE copyrighted material for advertising or promotional purposes or for creating new collective works for resale or redistribution, please go to htto://www.ieee,ora/publications standards/publications/riahts/riahts link.html to learn how to obtain a License from Rights Link.

If applicable, University Microfilms and/or ProQuest Library, or the Archives of Canada may supply single copies of the dissertation. 


\section{REFERENCES}

Acharya, U. R., Joseph, K. P., Kannathal, N., Lim, C. M. \& Suri, J. S. 2006. Heart rate variability: A review. Medical and Biological Engineering and Computing, 44, 1031-1051.

Alexandridis, E., Argyropoulos, T. \& Krastel, H. 1981. The latent period of the pupil light reflex in lesions of the optic nerve. Ophthalmologica, 182, 211-217.

American Psychiatric Association, 2000. Diagnostic and statistical manual of mental disorders, Washington, DC: American Psychiatric Association. Text Revision.

Anderson, C. J. \& Colombo, J. 2009. Larger tonic pupil size in young children with autism spectrum disorder. Developmental Psychobiology, 51, 207-211.

Anderson, C., Colombo, J. \& Shaddy, D. J. 2006. Visual scanning and pupillary responses in young children with Autism Spectrum Disorder. Journal of Clinical and Experimental Neuropsychology, 28, 1238-1256.

Anderson, C. J., Colombo, J. \& Unruh, K. E. 2012. Pupil and salivary indicators of autonomic dysfunction in autism spectrum disorder. Developmental Psychobiology, (in press).

Appenzeller, O. 1999. The Autonomic Nervous System Part I. Normal Functions, Elsevier.

Bakes, A., Bradshaw, C. M. \& Szabadi, E. 1990. Attention of the pupillary light reflex in anxious patients. British Journal of Clinical Pharmacology, 30, 377-381.

Bal, E., Harden, E., Lamb, D., Van Hecke, A. V., Denver, J. W. \& Porges, S. W. 2010. Emotion recognition in children with autism spectrum disorders: Relations to eye gaze and autonomic state. Journal of Autism and Developmental Disorders, 40, 358-370.

Bär, K. J., Boettger, M. K., Koschke, M., Schulz, S., Chokka, P., Yeragani, V. K. \& Voss, A. 2007. Non-linear complexity measures of heart rate variability in acute schizophrenia. Clinical Neurophysiology, 118, 2009-2015.

Bär, K. J., Boettger, M. K., Schulz, S., Harzendorf, C., Agelink, M. W., Yeragani, V. K., Chokka, P. \& Voss, A. 2008. The interaction between pupil function and cardiovascular 
regulation in patients with acute schizophrenia. Clinical Neurophysiology, 119, 22092213.

Bär, K. J., Letzsch, A., Jochum, T., Wagner, G., Greiner, W. \& Sauer, H. 2005. Loss of efferent vagal activity in acute schizophrenia. Journal of Psychiatric Research, 39, 519527.

Bär, K. J., Schulz, S., Koschke, M., Harzendorf, C., Gayde, S., Berg, W., Voss, A., Yeragani, V. K. \& Boettger, M. K. 2009. Correlations between the autonomic modulation of heart rate, blood pressure and the pupillary light reflex in healthy subjects. Journal of the Neurological Sciences, 279, 9-13.

Barbur, J. L. 2004. Learning from the pupil - Studies of basic mechanisms and clinical applications. In: WERNER, L. M. C. A. J. S. (ed.) The Visual Neurosciences. MIT Press.

Bashat, B. D., Kronfeld-Duenias, V., Zachor, D. A., Ekstein, P. M., Hendler, T., Tarrasch, R., Even, A., Levy, Y. \& Ben Sira, L. 2007. Accelerated maturation of white matter in young children with autism: A high b value DWI study. Neurolmage, 37, 40-47.

Bashat, D. B., Sira, L. B., Graif, M., Pianka, P., Hendler, T., Cohen, Y. \& Assaf, Y. 2005. Normal white matter development from infancy to adulthood: Comparing diffusion tensor and high $b$ value diffusion weighted MR images. Journal of Magnetic Resonance Imaging, 21, 503-511.

Bernardi, L., Spallone, V., Stevens, M., Hilsted, J., Frontoni, S., Pop-Busui, R., Ziegler, D., Kempler, P., Freeman, R., Low, P., Tesfaye, S. \& Valensi, P. 2011. Methods of investigation for cardiac autonomic dysfunction in human research studies. Diabetes/Metabolism Research and Reviews, 27, 654-664.

Berntson, G. G., Thomas Bigger Jr, J., Eckberg, D. L., Grossman, P., Kaufmann, P. G., Malik, M., Nagaraja, H. N., Porges, S. W., Saul, J. P., Stone, P. H. \& Van Der Molen, M. W. 1997. Heart rate variability: Origins methods, and interpretive caveats. Psychophysiology, 34, 623-648.

Berson, D. M. 2007. Phototransduction in ganglion-cell photoreceptors. Pflugers Archiv European Journal of Physiology, 454, 849-855. 
Bitsios, P., Prettyman, R. \& Szabadi, E. 1996. Changes in autonomic function with age: A study of pupillary kinetics in healthy young and old people. Age and Ageing, 25, 432438.

Bitsios, P., Szabadi, E. \& Bradshaw, C. M. 1999. Comparison of the effects of venlafaxine, paroxetine and desipramine on the pupillary light reflex in man. Psychopharmacology, $143,286-292$.

Bonnet, M. H. \& Arand, D. L. 1998. Heart rate variability in insomniacs and matched normal sleepers. Psychosomatic Medicine, 60, 610-615.

Boyd, B. A., McBee, M., Holtzclaw, T., Baranek, G. T. \& Bodfish, J. W. 2009. Relationships among repetitive behaviors, sensory features, and executive functions in high functioning autism. Research in Autism Spectrum Disorders, 3, 959-966.

Braune, H. J. \& Geisendörfer, U. 1995. Measurement of heart rate variations: Influencing factors, normal values and diagnostic impact on diabetic autonomic neuropathy. Diabetes Research and Clinical Practice, 29, 179-187.

Bremner, F. 2009. Pupil evaluation as a test for autonomic disorders. Clinical Autonomic Research, 19, 88-101.

Brennan, M., Palaniswami, M. \& Kamen, P. 2001. Do existing measures of Poincare plot geometry reflect nonlinear features of heart rate variability? Biomedical Engineering, IEEE Transactions on, 48, 1342-1347.

Carrillo-De-La-Peña, M., Rodríguez Holguín, S., Corral, M. \& Cadaveira, F. 1999. The effects of stimulus intensity and age on visual-evoked potentials (VEPs) in normal children. Psychophysiology, 36, 693-698.

Chan, H. L., Lin, M. A., Chao, P. K. \& Lin, C. H. 2007. Correlates of the shift in heart rate variability with postures and walking by time-frequency analysis. Computer Methods and Programs in Biomedicine, 86, 124-130.

Chaney, R. H., Givens, C. A., Aoki, M. F. \& Gombiner, M. L. 1989. Pupillary responses in recognizing awareness in persons with profound mental retardation. Perceptual and Motor Skills, 69, 523-528. 
Chen, Y. H., Rodgers, J. \& McConachie, H. 2009. Restricted and repetitive behaviours, sensory processing and cognitive style in children with autism spectrum disorders. Journal of Autism and Developmental Disorders, 39, 635-642.

Clarke, R. J. 2007. Shaping the pupil's response to light in the hooded rat. Experimental Brain Research, 176, 641-651.

Cocker, K. D., Fielder, A. R., Moseley, M. J. \& Edwards, A. D. 2005. Measurements of pupillary responses to light in term and preterm infants. Neuro-Ophthalmology, 29, 95101.

Cohen, J. 1992. A power primer. Psychological Bulletin, 112, 155-159.

Constantino, J. N. \& Gruber, C. P. 2005. The social responsiveness scale (SRS) manual, Los Angeles: Western Psychological Services.

Dalsgaard, S. 2012. Attention-deficit/hyperactivity disorder (ADHD). European Child and Adolescent Psychiatry, 1-6.

Daluwatte, C., Miles, J. H. \& Yao, G. 2012a. Simultaneously measured pupillary light reflex and heart rate variability in healthy children. Physiological Measurement, 33, 1043-1052.

Daluwatte, C., Miles, J. H., Christ, S. E., Beversdorf, D. Q., Takahashi, T. N. \& Yao, G. 2012b. Atypical Pupillary Light Reflex and Heart Rate Variability in Children with Autism Spectrum Disorder. Journal of Autism and Developmental Disorders, 1-16.

Daneault, V., Vandewalle, G., Hébert, M., Teikari, P., Mure, L. S., Doyon, J., Gronfier, C., Cooper, H. M., Dumont, M. \& Carrier, J. 2012. Does pupil constriction under blue and green monochromatic light exposure change with age? Journal of Biological Rhythms, $27,257-264$.

Delaney, J. P. A. \& Brodie, D. A. 2000. Effects of short-term psychological stress on the time and frequency domains of heart-rate variability. Perceptual and Motor Skills, 91, 515-524. 
Dockstader, C., Gaetz, W., Rockel, C. \& Mabbott, D. J. 2012. White matter maturation in visual and motor areas predicts the latency of visual activation in children. Human Brain Mapping, 33, 179-191.

Duby, J. C., Lipkin, P. H., Macias, M. M., Wegner, L. M., Duncan, P., Hagan Jr, J. F., Cooley, W. C., Swigonski, N., Biondich, P. G., Lollar, D., Ackermann, J., Brin, A., Crane, M., Gibson, A., Skipper, S. M., Steinberg-Hastings, D. \& Capers, M. 2006. Identifying infants and young children with developmental disorders in the medical home: An algorithm for developmental surveillance and screening. Pediatrics, 118, 405-420.

Dunn, W. 1999. The Sensory Profile Manual, The Psychological Corporation, San Antonio.

Dunn, W. 2002. The Infant/Toddler Sensory Profile Manual, The Psychological Corporation, San Antonio.

Dupuy, O., Mekary, S., Berryman, N., Bherer, L., Audiffren, M. \& Bosquet, L. 2012. Reliability of heart rate measures used to assess post-exercise parasympathetic reactivation. Clinical Physiology and Functional Imaging, 32, 296-304.

Dütsch, M., Marthol, H., Michelson, G., Neundörfer, B. \& Hilz, M. J. 2004. Pupillography refines the diagnosis of diabetic autonomic neuropathy. Journal of the Neurological Sciences, 222, 75-81.

Eaves, L. C., Wingert, H. D., Ho, H. H. \& Mickelson, E. C. R. 2006. Screening for autism spectrum disorders with the social communication questionnaire. Journal of Developmental and Behavioral Pediatrics, 27, S95-S103.

Eckberg, D. L. 1997. Sympathovagal balance: A critical appraisal. Circulation, 96, 32243232.

Eilers, H. \& Larson, M. D. 2010. The effect of ketamine and nitrous oxide on the human pupillary light reflex during general anesthesia. Autonomic Neuroscience: Basic and Clinical, 152, 108-114.

Fabrigar, L. R., MacCallum, R. C., Wegener, D. T. \& Strahan, E. J. 1999. Evaluating the use of exploratory factor analysis in psychological research. Psychological Methods, 4, 272299. 
Fan, X. \& Yao, G. 2011. Modeling transient pupillary light reflex induced by a short light flash. IEEE Transactions on Biomedical Engineering, 58, 36-42.

Fan, X., Miles, J. H., Takahashi, N. \& Yao, G. 2009a. Abnormal transient pupillary light reflex in individuals with autism spectrum disorders. Journal of Autism and Developmental Disorders, 39, 1499-1508.

Fan, X., Hearne, L., Lei, B., Miles, J. H., Takahashi, N. \& Yao, G. 2009b. Weak gender effects on transient pupillary light reflex. Autonomic Neuroscience: Basic and Clinical, 147, 9-13.

Fitzgibbon, A., Pilu, M. \& Fisher, R. B. 1999. Direct least square fitting of ellipses. Pattern Analysis and Machine Intelligence, IEEE Transactions on, 21, 476-480.

Fotiou, D. F., Brozou, C. G., Haidich, A. B., Tsiptsios, D., Nakou, M., Kabitsi, A., Giantselidis, C. \& Fotiou, F. 2007a. Pupil reaction to light in Alzheimer's disease: Evaluation of pupil size changes and mobility. Aging - Clinical and Experimental Research, 19, 364-371.

Fotiou, D. F., Brozou, C. G., Tsiptsios, D. J., Fotiou, A., Kabitsi, A., Nakou, M., Giantselidis, C. \& Goula, A. 2007b. Effect of age on pupillary light reflex: Evaluation of pupil mobility for clinical practice and research. Electromyography and Clinical Neurophysiology, 47, 11-22.

Fotiou, F., Fountoulakis, K. N., Goulas, A., Alexopoulos, L. \& Palikaras, A. 2000. Automated standardized pupillometry with optical method for purposes of clinical practice and research. Clinical Physiology, 20, 336-347.

Fountoulakis, K., Fotiou, F., lacovides, A., Tsiptsios, J., Goulas, A., Tsolaki, M. \& lerodiakonou, C. 1999. Changes in pupil reaction to light in melancholic patients. International Journal of Psychophysiology, 31, 121-128.

Fuentes, C. T., Mostofsky, S. H. \& Bastian, A. J. 2011. No proprioceptive deficits in autism despite movement-related sensory and execution impairments. Journal of Autism and Developmental Disorders, 41, 1352-1361. 
Gamelin, F. X., Berthoin, S. \& Bosquet, L. 2006. Validity of the polar S810 Heart rate monitor to measure R-R intervals at rest. Medicine and Science in Sports and Exercise, $38,887-893$.

Gamelin, F. X., Baquet, G., Berthoin, S. \& Bosquet, L. 2008. Validity of the polar S810 to measure R-R intervals in children. International Journal of Sports Medicine, 29, 134-138.

Geschwind, D. H. \& Levitt, P. 2007. Autism spectrum disorders: developmental disconnection syndromes. Current Opinion in Neurobiology, 17, 103-111.

Giza, E., Fotiou, D., Bostantjopoulou, S., Katsarou, Z. \& Karlovasitou, A. 2011. Pupil light reflex in Parkinson's disease: Evaluation with pupillometry. International Journal of Neuroscience, 121, 37-43.

Goodie, J. L., Larkin, K. T. \& Schauss, S. 2000. Validation of the Polar heart rate monitor for assessing heart rate during physical and mental stress. Journal of Psychophysiology, $14,159-164$.

Huikuri, H. V., Mäkikallio, T. H., Peng, C. K., Goldberger, A. L., Hintze, U. \& Møller, M. 2000. Fractal correlation properties of R-R interval dynamics and mortality in patients with depressed left ventricular function after an acute myocardial infarction. Circulation, 101, 47-53.

Hultborn, H., Mori, K. \& Tsukahara, N. 1978. Cerebellar influence of parasympathetic neurones innervating intra-ocular muscles. Brain Research, 159, 269-278.

Ijichi, Y., Kiyohara, T., Hosoba, M. \& Tsukahara, N. 1977. The cerebellar control of the pupillary light reflex in the cat. Brain Research, 128, 69-79.

Jones, C. R. G., Happé, F., Baird, G., Simonoff, E., Marsden, A. J. S., Tregay, J., Phillips, R. J., Goswami, U., Thomson, J. M. \& Charman, T. 2009. Auditory discrimination and auditory sensory behaviours in autism spectrum disorders. Neuropsychologia, 47, 28502858.

Kaeser, P. F. \& Kawasaki, A. 2010. Disorders of Pupillary Structure and Function. Neurologic Clinics, 28, 657-677. 
Kaltsatou, A., Kouidi, E., Fotiou, D. \& Deligiannis, P. 2011. The use of pupillometry in the assessment of cardiac autonomic function in elite diVerent type trained athletes. European Journal of Applied Physiology, 111, 2079-2087.

Kamath, M. V. \& Fallen, E. L. 1993. Power spectral analysis of heart rate variability: A noninvasive signature of cardiac autonomic function. Critical Reviews in Biomedical Engineering, 21, 245-311.

Katona, P. G., McLean, M., Dighton, D. H. \& Guz, A. 1982. Sympathetic and parasympathetic cardiac control in athletes and nonathletes at rest. Journal of Applied Physiology Respiratory Environmental and Exercise Physiology, 52, 1652-1657.

Kawasaki, A. \& Kardon, R. H. 2007. Intrinsically photosensitive retinal ganglion cells. Journal of Neuro-Ophthalmology, 27, 195-204.

Kientz, M. A. \& Dunn, W. 1997. A Comparison of the Performance of Children With and Without Autism on the Sensory Profile. American Journal of Occupational Therapy, 51, 530-537.

Kline, P. 2000. Handbook of Psychological Testing, Routledge.

Klintwall, L., Holm, A., Eriksson, M., Carlsson, L. H., Olsson, M. B., Hedvall, Å., Gillberg, C. \& Fernell, E. 2011. Sensory abnormalities in autism. A brief report. Research in Developmental Disabilities, 32, 795-800.

Kohnen, E. M., Zubcov, A. A. \& Kohnen, T. 2004. Scotopic pupil size in a normal pediatric population using infrared pupillometry. Graefe's Archive for Clinical and Experimental Ophthalmology, 242, 18-23.

Kojima, M., Shioiri, T., Hosoki, T., Kitamura, H., Bando, T. \& Someya, T. 2004. Pupillary light reflex in panic disorder: A trial using audiovisual stimulation. European Archives of Psychiatry and Clinical Neuroscience, 254, 242-244.

Kootz, J. P. \& Cohen, D. J. 1981. Modulation of sensory intake in autistic children. Cardiovascular and behavioral indices. Journal of the American Academy of Child Psychiatry, 20, 692-701. 
Krishnan, B., Jeffery, A., Metcalf, B., Hosking, J., Voss, L., Wilkin, T. \& Flanagan, D. E. 2009. Gender differences in the relationship between heart rate control and adiposity in young children: A cross-sectional study (EarlyBird 33). Pediatric Diabetes, 10, 127-134.

La Fountaine, M. F., Wecht, J. M., Spungen, A. M. \& Bauman, W. A. 2010. Intra-inter visit reproducibility of short-term linear and nonlinear measurement of heart rate variability in tetraplegia and neurologically intact controls. Physiological Measurement, 31, 363374.

Laeng, B., Sirois, S. \& Gredebäck, G. 2012. Pupillometry: A window to the preconscious? Perspectives on Psychological Science, 7, 18-27.

Lenassi, E., Likar, K., Stirn-Kranjc, B. \& Brecelj, J. 2008. VEP maturation and visual acuity in infants and preschool children. Documenta Ophthalmologica, 117, 111-120.

Levy, M. N. 1990. Autonomic interactions in cardiac control. Annals of the New York Academy of Sciences, 601, 209-221.

Levy, M. N. 1997. Neural control of cardiac function. Bailliere's Clinical Neurology, 6, 227-244.

Loewenfeld, I. E. 1999. The pupil: Anatomy, physiology, and clinical applications. Detroit: Wayne State University Press.

Lombardi, F., Mäkikallio, T. H., Myerburg, R. J. \& Huikuri, H. V. 2001. Sudden cardiac death: Role of heart rate variability to identify patients at risk. Cardiovascular Research, $50,210-217$.

Lord, C., Rutter, M. \& Couteur, A. L. 1994. Autism diagnostic interview-revised: A revised version of a diagnostic interview for caregivers of individuals with possible pervasive developmental disorders. Journal of Autism and Developmental Disorders, 24, 659-685.

Lord, C., Rutter, M., Goode, S., Heemsbergen, J., Jordan, H., Mawhood, L. \& Schopler, E. 1989. Autism diagnostic observation schedule: A standardized observation of communicative and social behavior. Journal of Autism and Developmental Disorders, 19, 185-212. 
Lowenstein, O. \& Loewenfeld, I. E. 1950. Mutual role of sympathetic and parasympathetic in shaping of the pupillary reflex to light; pupillographic studies. Archives of neurology and psychiatry, 64, 341-377.

Mabbott, D. J., Noseworthy, M., Bouffet, E., Laughlin, S. \& Rockel, C. 2006. White matter growth as a mechanism of cognitive development in children. Neurolmage, 33, 936-946.

MacCallum, R. C., Widaman, K. F., Zhang, S. \& Hong, S. 1999. Sample size in factor analysis. Psychological Methods, 4, 84-99.

Maclean, H. \& Dhillon, B. 1993. Pupil cycle time and human immunodeficiency virus (HIV) infection. Eye, 7, 785-786.

Malik, M. \& Eckberg, D. L. 1998. Sympathovagal balance: A critical appraisal [3] (multiple letters). Circulation, 98, 2643-2644.

Malik, M., Camm, A. J., Bigger Jr, J. T., Breithardt, G., Cerutti, S., Cohen, R. J., Coumel, P., Fallen, E. L., Kennedy, H. L., Kleiger, R. E., Lombardi, F., Malliani, A., Moss, A. J., Rottman, J. N., Schmidt, G., Schwartz, P. J. \& Singer, D. H. 1996. Heart rate variability. Standards of measurement, physiological interpretation, and clinical use. European Heart Journal, 17, 354-381.

Malliani, A., Lombardi, F. \& Pagani, M. 1994. Power spectrum analysis of heart rate variability: A tool to explore neural regulatory mechanisms. British Heart Journal, 71, 12.

Malliani, A., Pagani, M., Lombardi, F. \& Cerutti, S. 1991. Cardiovascular neural regulation explored in the frequency domain. Circulation, 84, 482-492.

Martineau, J., Hernandez, N., Hiebel, L., Roché, L., Metzger, A. \& Bonnet-Brilhault, F. 2011. Can pupil size and pupil responses during visual scanning contribute to the diagnosis of autism spectrum disorder in children? Journal of Psychiatric Research, 45, 1077-1082.

Massin, M. \& von Bernuth, G. 1997. Normal ranges of heart rate variability during infancy and childhood. Pediatric Cardiology, 18, 297-302. 
Mathewson, K. J., Drmic, I. E., Jetha, M. K., Bryson, S. E., Goldberg, J. O., Hall, G. B., Santesso, D. L., Segalowitz, S. J. \& Schmidt, L. A. 2011. Behavioral and cardiac responses to emotional stroop in adults with autism spectrum disorders: Influence of medication. Autism Research, 4, 98-108.

McCulloch, D. L. \& Skarf, B. 1991. Development of the human visual system: Monocular and binocular pattern VEP latency. Investigative Ophthalmology and Visual Science, 32, 2372-2381.

Ming, X., Julu, P. O. O., Brimacombe, M., Connor, S. \& Daniels, M. L. 2005. Reduced cardiac parasympathetic activity in children with autism. Brain and Development, 27, 509-516.

Ming, X., Bain, J. M., Smith, D., Brimacombe, M., Gold Von-Simson, G. \& Axelrod, F. B. 2011. Assessing autonomic dysfunction symptoms in children: A pilot study. Journal of Child Neurology, 26, 420-427.

Montano, N., Ruscone, T., Porta, A., Lombardi, F., Pagani, M. \& Malliani, A. 1994. Power spectrum analysis of heart rate variability to assess the changes in sympathovagal balance during graded orthostatic tilt. Circulation, 90, 1826-1831.

Mukai, S. \& Hayano, J. 1995. Heart rate and blood pressure variabilities during graded head-up tilt. Journal of Applied Physiology, 78, 212-216.

Murray, M. J. 2010. Attention-deficit/hyperactivity disorder in the context of autism spectrum disorders. Current Psychiatry Reports, 12, 382-388.

Musser, E. D., Backs, R. W., Schmitt, C. F., Ablow, J. C., Measelle, J. R. \& Nigg, J. T. 2011. Emotion regulation via the autonomic nervous system in children with attentiondeficit/hyperactivity disorder (ADHD). Journal of Abnormal Child Psychology, 39, 841852.

Nakagawa, S. \& Cuthill, I. C. 2007. Effect size, confidence interval and statistical significance: A practical guide for biologists. Biological Reviews, 82, 591-605.

Neuhuber, W. \& Schrödl, F. 2011. Autonomic control of the eye and the iris. Autonomic Neuroscience: Basic and Clinical, 165, 67-79. 
Nunan, D., Gay, D., Jakovljevic, D. G., Hodges, L. D., Sandercock, G. R. H. \& Brodie, D. A. 2009. Validity and reliability of short-term heart-rate variability from the Polar S810. Medicine and Science in Sports and Exercise, 41, 243-250.

Pagani, M. 2000. Heart rate variability and autonomic diabetic neuropathy. Diabetes, Nutrition and Metabolism - Clinical and Experimental, 13, 341-346.

Pagani, M., Lombardi, F. \& Guzzetti, S. 1986. Power spectral analysis of heart rate and arterial pressure variabilities as a marker of sympatho-vagal interaction in man and conscious dog. Circulation Research, 59, 178-193.

Palkovitz, R. J. \& Wiesenfeld, A. R. 1980. Differential autonomic responses of autistic and normal children. Journal of Autism and Developmental Disorders, 10, 347-360.

Panda, S., Nayak, S. K., Campo, B., Walker, J. R., Hogenesch, J. B. \& Jegla, T. 2005. Illumination of the melanopsin signaling pathway. Science, 307, 600-604.

Peng, C. K., Havlin, S., Stanley, H. E. \& Goldberger, A. L. 1995. Quantification of scaling exponents and crossover phenomena in nonstationary heartbeat time series. Chaos, 5 , 82-87.

Porto, L. G. G. \& Junqueira Jr, L. F. 2009. Comparison of time-domain short-term heart interval variability analysis using a wrist-worn heart rate monitor and the conventional electrocardiogram. PACE - Pacing and Clinical Electrophysiology, 32, 43-51.

Raven, J., Raven, J. C. \& Court, J. H. 1996. Manual for Raven's progressive matrices and vocabulary scales, Oxford: Oxford Psychologists Press.

Robertson, D. 2012. Primer on the autonomic nervous system, Elsevier.

Rubin, L. S. 1961. Patterns of pupillary dilatation and constriction in psychotic adults and autistic children. Journal of Nervous and Mental Disease, 133, 130-142.

Russolillo, G. \& Lauro, C. N. 2011. A Proposal for Handling Categorical Predictors in PLS Regression Framework Classification and Multivariate Analysis for Complex Data Structures. In: FICHET, B., PICCOLO, D., VERDE, R. \& VICHI, M. (eds.). Springer Berlin Heidelberg. 
Sacks, B. \& Smith, S. 1989. People with Down's syndrome can be distinguished on the basis of cholinergic dysfunction. Journal of Neurology Neurosurgery and Psychiatry, 52, 1294-1295.

Salo, T. M., Voipio-Pulkki, L. M., Jalonen, J. O., Helenius, H., Viikari, J. S. A. \& Kantola, I. 1999. Reproducibility of abnormal heart rate variability indices: The case of hypertensive sleep apnoea syndrome. Clinical Physiology, 19, 258-268.

Sand, N., Silverstein, M., Glascoe, F. P., Gupta, V. B., Tonniges, T. P. \& O'Connor, K. G. 2005. Pediatricians' Reported Practices Regarding Developmental Screening: Do Guidelines Work? Do They Help? Pediatrics, 116, 174-179.

Saper, C. B. 2002. The central autonomic nervous system: Conscious visceral perception and autonomic pattern generation. Annual Review of Neuroscience, 25, 433-469.

Silvetti, M. S., Drago, F. \& Ragonese, P. 2001. Heart rate variability in healthy children and adolescents is partially related to age and gender. International Journal of Cardiology, 81, 169-174.

Simpson, J. I., Giolli, R. A. \& Blanks, R. H. 1988. The pretectal nuclear complex and the accessory optic system. Reviews of oculomotor research, 2, 335-364.

Slooter, J. \& Van Norren, D. 1980. Visual acuity measured with pupil responses to checkerboard stimuli. Investigative Ophthalmology and Visual Science, 19, 105-108.

Smith, S. A. \& Smith, S. E. 1983. Reduced pupillary light reflexes in diabetic autonomic neuropathy. Diabetologia, 24, 330-332.

Sokolski, K. N. \& Demet, E. M. 1996. Increased pupillary sensitivity to pilocarpine in depression. Progress in Neuro-Psychopharmacology and Biological Psychiatry, 20, 253261.

Stergiou, V., Fotiou, D., Tsiptsios, D., Haidich, B., Nakou, M., Giantselidis, C. \& Karlovasitou, A. 2009. Pupillometric findings in patients with Parkinson's disease and cognitive disorder. International Journal of Psychophysiology, 72, 97-101. 
Steyn, H. S. \& Ellis, S. M. 2009. Estimating an effect size in one-way multivariate analysis of variance (MANOVA). Multivariate Behavioral Research, 44, 106-129.

Thayer, J. F. \& Sternberg, E. 2006. Beyond heart rate variability: Vagal regulation of allostatic systems. Annals of the New York Academy of Sciences, 1088, 361-372.

Theofilopoulos, N., McDade, G., Szabadi, E. \& Bradshaw, C. M. 1995. Effects of reboxetine and desipramine on the kinetics of the pupillary light reflex. British Journal of Clinical Pharmacology, 39, 251-255.

Toichi, M. \& Kamio, Y. 2003. Paradoxical autonomic response to mental tasks in autism. Journal of Autism and Developmental Disorders, 33, 417-426.

Tomchek, S. D. \& Dunn, W. 2007. Sensory processing in children with and without autism: a comparative study using the short sensory profile. American Journal of Occupational Therapy, 61, 190-200.

Troger, J., Kieselbach, G., Teuchner, B., Kralinger, M., Nguyen, Q. A., Haas, G., Yayan, J., Göttinger, W. \& Schmid, E. 2007. Peptidergic nerves in the eye, their source and potential pathophysiological relevance. Brain Research Reviews, 53, 39-62.

Tsukahara, N., Kiyohara, T. \& Ijichi, Y. 1973. The mode of cerebellar control of pupillary light reflex. Brain Research, 60, 244-248.

Ukai, K. 1985. Spatial pattern as a stimulus to the pupillary system. Journal of the Optical Society of America. A, Optics and Image Science, 2, 1094-1100.

Umetani, K., Singer, D. H., McCraty, R. \& Atkinson, M. 1998. Twenty-four hour time domain heart rate variability and heart rate: Relations to age and gender over nine decades. Journal of the American College of Cardiology, 31, 593-601.

Vallbo, A. B., Hagbarth, K. E., Torebjork, H. E. \& Wallin, B. G. 1979. Somatosensory, proprioceptive, and sympathetic activity in human peripheral nerves. Physiological Reviews, 59, 919-957. 
Van Diemen, H. A. M., Van Dongen, M. M. M. M., Nauta, J. J. P., Lanting, P. \& Polman, C. H. 1992. Pupillary light reflex latency in patients with multiple sclerosis. Electroencephalography and Clinical Neurophysiology, 82, 213-219.

van Engeland, H., Roelofs, J. W., Verbaten, M. N. \& Slangen, J. L. 1991. Abnormal electrodermal reactivity to novel visual stimuli in autistic children. Psychiatry Research, 38, 27-38.

van Lang, N. D. J., Tulen, J. H. M., Kallen, V. L., Rosbergen, B., Dieleman, G. \& Ferdinand, R. F. 2007. Autonomic reactivity in clinically referred children attention-deficit/ hyperactivity disorder versus anxiety disorder. European Child and Adolescent Psychiatry, 16, 71-78.

Vissers, M. E., X Cohen, M. \& Geurts, H. M. 2012. Brain connectivity and high functioning autism: A promising path of research that needs refined models, methodological convergence, and stronger behavioral links. Neuroscience and Biobehavioral Reviews, $36,604-625$.

Wagner, J. B., Hirsch, S. B., Vogel-Farley, V. K., Redcay, E. \& Nelson, C. A. 2012. EyeTracking, Autonomic, and Electrophysiological Correlates of Emotional Face Processing in Adolescents with Autism Spectrum Disorder. Journal of Autism and Developmental Disorders, 1-12.

Wechsler, D. 1991. Wechsler intelligence scale for children $3^{\text {rd }}$ edition manual. New York: The Psychological Corporation.

Weinstein, M., Ben-Sira, L., Levy, Y., Zachor, D. A., Itzhak, E. B., Artzi, M., Tarrasch, R., Eksteine, P. M., Hendler, T. \& Bashat, D. B. 2011. Abnormal white matter integrity in young children with autism. Human Brain Mapping, 32, 534-543.

Winn, B., Whitaker, D., Elliott, D. B. \& Phillips, N. J. 1994. Factors affecting light-adapted pupil size in normal human subjects. Investigative Ophthalmology and Visual Science, $35,1132-1137$.

Woodard, C. R., Goodwin, M. S., Zelazo, P. R., Aube, D., Scrimgeour, M., Ostholthoff, T. \& Brickley, M. 2012. A comparison of autonomic, behavioral, and parent-report measures of sensory sensitivity in young children with autism. Research in Autism Spectrum Disorders, 6, 1234-1246. 
Xu, L., Oja, E. \& Kultanen, P. 1990. A new curve detection method: Randomized Hough transform (RHT). Pattern Recognition Letters, 11, 331-338.

Yen, J. 1956. On Nonuniform Sampling of Bandwidth-Limited Signals. IRE Transactions on Circuit Theory, 3, 251-257.

Yeragani, V. K., Pohl, R., Berger, R., Balon, R., Ramesh, C., Glitz, D., Srinivasan, K. \& Weinberg, P. 1993. Decreased heart rate variability in panic disorder patients: A study of power-spectral analysis of heart rate. Psychiatry Research, 46, 89-103.

Young, R. S. \& Alpern, M. 1980. Pupil responses to foveal exchange of monochromatic lights. Journal of the Optical Society of America, 70, 697-706.

Young, R. S. L., Han, B. C. \& Wu, P. Y. 1993. Transient and sustained components of the pupillary responses evoked by luminance and color. Vision Research, 33, 437-446.

Zoghbi, H. Y. \& Bear, M. F. 2012. Synaptic dysfunction in neurodevelopmental disorders associated with autism and intellectual disabilities. Cold Spring Harbor Perspectives in Biology, 4. 


\section{VITA}

Chathuri Daluwatte was born on October $7^{\text {th }}, 1983$ in Colombo, Sri Lanka. She received her bachelor's degree in electronic and telecommunication engineering in November, 2007 from University of Moratuwa in Moratuwa, Sri Lanka. In December 2012, she earned her M.S. in statistics from University of Missouri-Columbia. She started her Ph.D. study in biological engineering at University of Missouri-Columbia in January, 2009. Her research interests include biomedical instrumentation, image processing, and physiological and neurological measurements. 